TRANSACTIONS OF THE

AMERICAN MATHEMATICAL SOCIETY

Volume 360, Number 10, October 2008, Pages 5291-5342

S 0002-9947(08)04468-1

Article electronically published on February 27, 2008

\title{
STABILITY AND INSTABILITY \\ OF LIAPUNOV-SCHMIDT AND HOPF BIFURCATION \\ FOR A FREE BOUNDARY PROBLEM \\ ARISING IN A TUMOR MODEL
}

\author{
AVNER FRIEDMAN AND BEI HU
}

\begin{abstract}
We consider a free boundary problem for a system of partial differential equations, which arises in a model of tumor growth. For any positive number $R$ there exists a radially symmetric stationary solution with free boundary $r=R$. The system depends on a positive parameter $\mu$, and for a sequence of values $\mu_{2}<\mu_{3}<\cdots$ there also exist branches of symmetricbreaking stationary solutions, parameterized by $\varepsilon,|\varepsilon|$ small, which bifurcate from these values. In particular, for $\mu=\mu(\varepsilon)$ near $\mu_{2}$ the free boundary has the form $r=R+\varepsilon Y_{2,0}(\theta)+O\left(\varepsilon^{2}\right)$ where $Y_{2,0}$ is the spherical harmonic of mode $(2,0)$. It was recently proved by the authors that the stationary solution is asymptotically stable for any $0<\mu<\mu_{*}$, but linearly unstable if $\mu>\mu_{*}$, where $\mu_{*}=\mu_{2}$ if $R>\bar{R}$ and $\mu_{*}<\mu_{2}$ if $R<\bar{R} ; \bar{R} \approx 0.62207$. In this paper we prove that for $R>\bar{R}$ each of the stationary solutions which bifurcates from $\mu=\mu_{2}$ is linearly stable if $\varepsilon>0$ and linearly unstable if $\varepsilon<0$. We also prove, for $R<\bar{R}$, that the point $\mu=\mu_{*}$ is a Hopf bifurcation, in the sense that the linearized time-dependent problem has a family of solutions which are asymptotically periodic in $t$.
\end{abstract}

\section{Statement of the Problem}

Consider the following problem: Find 3-dimensional domains $\Omega(t)$ and functions $\sigma, p$ such that:

$$
\begin{aligned}
& \sigma_{t}-\Delta \sigma+\sigma=0 \quad \text { in } \Omega(t), t>0, \\
& \Delta p=-\mu(\sigma-\widetilde{\sigma}) \quad \text { in } \Omega(t), t>0 \quad(0<\widetilde{\sigma}<1), \\
& \sigma=1 \quad \text { on } \partial \Omega(t), t>0, \\
& p=\kappa \quad \text { on } \partial \Omega(t), t>0
\end{aligned}
$$

where $\kappa$ is the mean curvature $(\kappa>0$ if $\Omega(t)$ is a ball), and

$$
V_{n}=-\frac{\partial p}{\partial n} \quad \text { on } \partial \Omega(t), t>0
$$

Received by the editors February 24, 2005 and, in revised form, September 26, 2005 and September 6, 2006.

2000 Mathematics Subject Classification. Primary 35R35, 35K55; Secondary 35Q80, 35C20, $92 \mathrm{C} 37$.

Key words and phrases. Free boundary problems, stationary solution, stability, instability, bifurcation, symmetry-breaking, tumor cell.

(C)2008 American Mathematical Society Reverts to public domain 28 years from publication 
where $\partial / \partial n$ is the derivative along the outward normal $\vec{n}$ and $V_{n}$ is the velocity of the free boundary $\partial \Omega(t)$ in the direction $\vec{n}$, and

$$
\begin{gathered}
\partial \Omega(0): r=R_{0}(\theta, \phi), \\
\left.\sigma\right|_{t=0}=\sigma_{0}(r, \theta, \phi)
\end{gathered}
$$

are given initial conditions.

The system represents a simple model of tumor growth where the tumor region $\Omega(t)$ contains just one type of cells with density $c$; $\sigma$ is the concentration of nutrients, and $p$ is the pressure which results from cell proliferation. It is assumed that the cell density is linearly dependent on the concentration of nutrients, and, for simplicity, we take $c=\sigma$. It is also assumed in this model that the cell density grows according to the proliferation rate $\mu(\sigma-\widetilde{\sigma})$ where $\widetilde{\sigma}$ is a constant smaller than the concentration of nutrients on the boundary, that is, $\widetilde{\sigma}<1$.

Many mathematical models of tumor growth, which consider the tumor tissue as a density of proliferating cells, have been developed and studied in many papers; see [5, 6, 17, 8, 14, 15, 18, 19] and the references given there.

Assuming that the tumor region is a porous medium, so that Darcy's law $\vec{V}=$ $-\nabla p$ holds, the law of conservation of mass $\operatorname{div} \vec{V}=\mu(\sigma-\widetilde{\sigma})$ yields the equation (1.2). Furthermore, the continuity condition $\vec{V} \cdot \vec{n}=V_{n}$ yields the relation (1.5). Finally, the cell-to-cell adhesiveness suggests the relation (1.4) (see [2], 3], 4]).

It is known 14 that, under the previous assumption $0<\widetilde{\sigma}<1$, there exists a unique radially symmetric stationary solution $\left(\sigma_{S}(r), p_{S}(r), R_{S}\right)$, and it is given by

$$
\begin{gathered}
\sigma_{S}(r)=\frac{R_{S}}{\sinh R_{S}} \frac{\sinh r}{r}, \quad p_{S}(r)=C-\mu \sigma_{S}(r)+\frac{\mu}{6} \widetilde{\sigma} r^{2} \\
\text { where } C=\frac{1}{R_{S}}+\mu-\frac{\mu}{6} \widetilde{\sigma} R_{S}^{2},
\end{gathered}
$$

and $R_{S}$ is uniquely determined by the equation

$$
\frac{1}{R_{S}^{2}}\left(R_{S} \operatorname{coth} R_{S}-1\right)=\frac{\widetilde{\sigma}}{3}
$$

It was also proved in [10] that there exists a family of symmetry-breaking bifurcation branches of stationary solutions with free boundaries and $\mu$ 's given by

$$
r=R_{S}+\varepsilon Y_{n, 0}(\theta)+O\left(\varepsilon^{2}\right), \quad \mu=\mu_{n}+O(\varepsilon), \quad n \geq 2
$$

where $Y_{n, 0}(\theta)$ is the spherical harmonic of order $(n, 0),|\varepsilon|$ is small,

$$
\mu_{n}\left(R_{S}\right)=\frac{n[n(n+1)-2] I_{1 / 2}\left(R_{S}\right)}{2 R_{S}^{3} I_{3 / 2}\left(R_{S}\right)\left[I_{5 / 2}\left(R_{S}\right) / I_{3 / 2}\left(R_{S}\right)-I_{n+3 / 2}\left(R_{S}\right) / I_{n+1 / 2}\left(R_{S}\right)\right]},
$$

and $\mu_{n}\left(R_{S}\right)<\mu_{n+1}\left(R_{S}\right)$. We refer to these stationary bifurcations as LiapunovSchmidt bifurcations.

The terms $O\left(\varepsilon^{2}\right), O(\varepsilon)$ in (1.9) can actually be expanded into power series in $\varepsilon$. 
By asymptotic stability of the stationary solution (1.7) we mean the following: Given any initial values

$$
\begin{gathered}
R_{0}(\theta, \phi)=R_{S}+\eta \rho_{0}(\theta, \phi), \\
\sigma_{0}(r, \theta, \phi)=\sigma_{S}(r)+\eta w_{0}(r, \theta, \phi),
\end{gathered}
$$

if $|\eta|$ is sufficiently small, then there exists a solution to (1.1)-(1.6) for all $t>0$, and

$$
\partial \Omega(t) \rightarrow \text { sphere }|x-a|=R_{S} \quad \text { as } t \rightarrow \infty,
$$

for some center $a$; more precisely, as $t \rightarrow \infty$, the solution $(\sigma, p, \Omega(t))$ converges to the radially symmetric stationary solution centered at $a$.

Recently [12, 13] the authors have proved that the radially symmetric solutions (1.7) are asymptotically stable as long as

$$
0<\mu<\mu_{*}\left(R_{S}\right)
$$

where $\mu_{*}\left(R_{S}\right)$ is a function satisfying:

$$
\begin{array}{ll}
\mu_{*}\left(R_{S}\right)<\mu_{2}\left(R_{S}\right) & \text { if } R_{S}<\bar{R} \\
\mu_{*}\left(R_{S}\right)=\mu_{2}\left(R_{S}\right) & \text { if } R=\bar{R}
\end{array}
$$

and $\bar{R} \approx 0.62207$. It was also proved in 12 that the convergence in 1.12) (in case $\left.\mu<\mu_{*}\left(R_{S}\right)\right)$ is exponentially fast.

In addition to the concept of asymptotic stability, we introduce the concepts of linear stability and linear instability. We linearize the system (1.1)-(1.6) about $\left(\sigma_{S}, p_{S}, R_{S}\right)$ by writing

$$
\sigma=\sigma_{S}+\varepsilon w, \quad p=p_{S}+\varepsilon q, \quad R=R_{S}+\varepsilon \rho
$$

and collecting only the $\varepsilon$-order terms.

Because the original problem is invariant under translation of the origin, if we want to keep the origin fixed, we need to impose some constraints on the initial data. For the linearized problem these constraints are satisfied if

$$
\begin{aligned}
& \rho_{0}(\theta, \phi) \text { and } w_{0}(r, \theta, \phi) \text { do not contain spherical harmonics of } \\
& \text { order } 1: Y_{1, m}(\theta, \phi) \quad(m=-1,0,1) .
\end{aligned}
$$

In that case we say that the stationary solution $\left(\sigma_{S}, p_{S}, R_{S}\right)$ is linearly stable if $\rho(\theta, \phi, t) \rightarrow 0$ as $t \rightarrow \infty$. In the general case, by linear stability we mean that for any initial data $\rho_{0}(\theta, \phi)$ and $w_{0}(r, \theta, \phi)$,

$$
\rho(\theta, \phi, t)-\sum_{m=-1}^{1} a_{m} Y_{1, m}(\theta, \phi) \rightarrow 0 \quad \text { as } t \rightarrow \infty
$$

for some constants $a_{m}$; the $a_{m}$ vanish if (1.14) is satisfied.

If $\rho(\theta, \phi, t) \rightarrow \infty$ for a sequence $t=t_{m} \rightarrow \infty$, then we say $\left(\sigma_{S}, p_{S}, R_{S}\right)$ is linearly unstable.

In [12] we proved that if $\mu>\mu_{*}\left(R_{S}\right)$, then $\left(\sigma_{S}, p_{S}, R_{S}\right)$ is linearly unstable.

The concept of linear stability/instabilty can be defined with respect to any stationary solution, not just radially symmetric ones. In this paper we shall consider the linear stability/instability of the bifurcation branch (1.9) for $n=2$ in case $\mu_{*}\left(R_{S}\right)=\mu_{2}\left(R_{S}\right)$ and for a somewhat restricted version of the linearization 


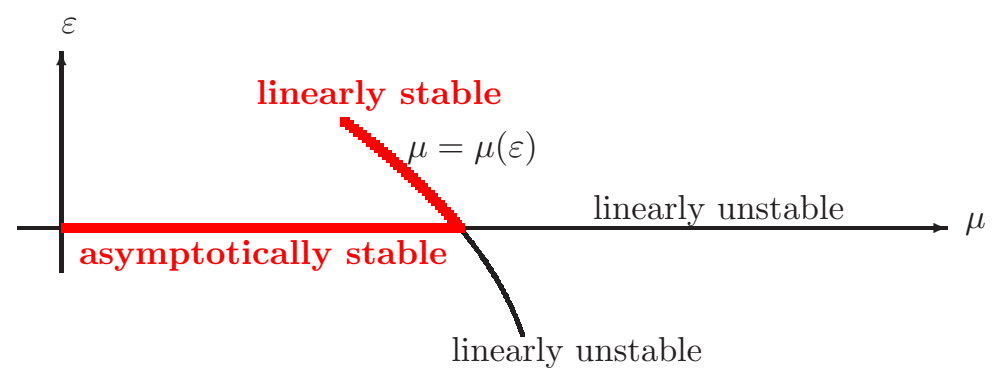

FiguRE 1. The free boundary is given by $r=R_{S}+\varepsilon Y_{2,0}+O\left(\varepsilon^{2}\right)$.

procedure, as will be explained in Section 5. We shall prove:

$$
\begin{aligned}
& \text { If } \mu_{*}\left(R_{S}\right)=\mu_{2}\left(R_{S}\right) \text {, then the stationary solutions (1.9) with } \varepsilon>0 \\
& \text { are linearly stable, and the solutions with } \varepsilon<0 \text { are linearly unstable. }
\end{aligned}
$$

Since we shall also prove that the $O(\varepsilon)$ term in the second equality in (1.9) is strictly monotone decreasing in $\varepsilon$, we can summarize the result (1.15) and the results of [12, 13] mentioned above in Figure 1]

Usually, one expects the bifurcation branches of the linear stability/instability in the diagram in Figure 1 reversed, namely, linear stability for $\mu(\varepsilon)>\mu_{2}$, instability for $\mu(\varepsilon)<\mu_{2}$. However, we note that the linear stability to be established in this paper is not the standard one since for each initial perturbation we also change the location of the origin of the nonlinear problem (see Theorem 10.1) and only then we linearize the system.

We shall also consider in this paper the case $\mu_{*}\left(R_{S}\right)<\mu_{2}\left(R_{S}\right)$ and prove the following Hopf bifurcation result:

If $\mu_{*}\left(R_{S}\right)<\mu_{2}\left(R_{S}\right)$, then there exist periodic solutions to the linearized problem with free boundary of the form $\rho(\theta, \phi, t)=$ $C_{1} \sin \beta t+C_{2} \cos \beta t+\sum_{m=-1}^{1} D_{m} Y_{1, m}(\theta, \phi)$; the constant $\beta$ is determined as a solution of a special transcendental equation. Furthermore, any solution to the linearized problem converges exponentially fast to one of these periodic solutions.

The first step in the proof of (1.15) is to compute the sign of the coefficient $\mu_{21}$ in the expansion

$$
\mu=\mu_{2}+\varepsilon \mu_{21}+O\left(\varepsilon^{2}\right) ;
$$

we shall prove that

$$
\mu_{21}<0 .
$$

In the process of proving (1.18) we shall give a new and simpler proof than 10 of the existence of the bifurcation branches (1.9), (1.10) for $n$ even. The proof uses various calculations from [12] and [10] as well as the following Crandall-Rabinowitz theorem. 
Theorem 1.1 (see [9, Theorem 1.7]). Let $X, Y$ be real Banach spaces and $F(x, \mu)$ a $C^{p}$ map, $p \geq 3$, of a neighborhood $\left(0, \mu_{0}\right)$ in $X \times \mathbb{R}$ into $Y$. Suppose

(i) $F(0, \mu)=0$ for all $\mu$ in a neighborhood of $\mu_{0}$,

(ii) $\operatorname{Ker} F_{x}\left(0, \mu_{0}\right)$ is a one dimensional space, spanned by $x_{0}$,

(iii) $\operatorname{Im} F_{x}\left(0, \mu_{0}\right)=Y_{1}$ has codimension 1 ,

(iv) $F_{\mu x}\left(0, \mu_{0}\right) x_{0} \notin Y_{1}$.

Then $\left(0, \mu_{0}\right)$ is a bifurcation point of the equation $F(x, \mu)=0$ in the following sense: In a neighborhood of $\left(0, \mu_{0}\right)$ the set of solutions of $F(x, \mu)=0$ consists of two $C^{p-2}$ smooth curves $\Gamma_{1}$ and $\Gamma_{2}$ which intersect only at the point $\left(0, \mu_{0}\right) ; \Gamma_{1}$ is the curve $(0, \mu)$ and $\Gamma_{2}$ can be parameterized as follows:

$$
\begin{aligned}
& \Gamma_{2}:(x(\varepsilon), \mu(\varepsilon)),|\varepsilon| \text { small, } \\
& (x(0), \mu(0))=\left(0, \mu_{0}\right), x^{\prime}(0)=x_{0} .
\end{aligned}
$$

In Section 2 we collect various formulas for the Bessel functions of the second kind $I_{m}(\xi)$, and for the spherical harmonic functions $Y_{n, m}(\theta, \phi)$. In Section 3 we prove the existence of symmetry breaking bifurcation branches of solutions as described in (1.9) - (1.10), using Theorem 1.1. To do that, we define an appropriate function $F$, compute its Fréchet derivatives, and derive various expansions around a stationary solution up to second order. In Section 4 we compute the coefficient $\mu_{21}$. In Section 5, we derive the linearized problem and in Section 6 we take its Laplace transform and formally obtain explicit formulas for the Laplace transform $\widehat{\rho}$ of the free boundary. We proceed in Section 7 to establish estimates on the coefficients $\widehat{\rho}_{n, 0}$ in the expansion

$$
\widehat{\rho}=\sum_{n=0}^{\infty} \widehat{\rho}_{n, 0} Y_{n, 0},
$$

and in Section 8 we justify the formal calculations of Section 6. Next, in Sections 9 and 10 we prove the instability and stability results for the first Liapunov-Schmidt bifurcation, as stated in (1.15). Finally, in Section 11 we prove the Hopf bifurcation result stated in (1.16).

Remark 1.1. The results of this paper extend immediately to the more general case where in (1.4) $\kappa$ is replaced by $\gamma \cdot \kappa$; in this case all the analysis remains the same except that the bifurcation parameter $\mu$ is replaced everywhere by $\mu / \gamma$. Our results also extend to the case where in (1.1) we replace $\sigma_{t}$ by $c \sigma_{t}$ and in (1.4) we replace $\kappa$ by $\gamma \cdot \kappa$. Indeed the transformation $t \rightarrow c \cdot \tau, c \cdot p \rightarrow p$ reduces this more general case to the case where (1.1), (1.3) hold, in (1.2) $\mu$ is replaced by $\mu / c$, and in (1.4) $\kappa$ is replaced by $\gamma / c$, so that again the bifurcation parameter is $\mu / \gamma$.

\section{Bessel FunCtions AND SPHERICAL HARMONiCS}

In the sequel we shall need several properties of the modified Bessel function $I_{m}(\xi)$, where $\xi$ is a complex variable and $m$ is any nonnegative real number. For convenience we collect all these facts in this section. The following relations are 
well known:

$$
\begin{aligned}
& I_{m}(\xi)=\left(\frac{\xi}{2}\right)^{m} \sum_{k=0}^{\infty} \frac{1}{k ! \Gamma(m+k+1)}\left(\frac{\xi}{2}\right)^{2 k}, \\
& I_{1 / 2}(\xi)=\sqrt{\frac{2}{\pi \xi}} \sinh \xi, \\
& I_{3 / 2}(\xi)=\sqrt{\frac{2}{\pi \xi}}\left\{-\frac{\sinh \xi}{\xi}+\cosh \xi\right\}, \\
& I_{5 / 2}(\xi)=\sqrt{\frac{2}{\pi \xi}}\left\{\left[\frac{3}{\xi^{2}}+1\right] \sinh \xi-\frac{3}{\xi} \cosh \xi\right\}, \\
& I_{m}^{\prime \prime}(\xi)+\frac{1}{\xi} I_{m}^{\prime}(\xi)-\left(1+\frac{m^{2}}{\xi^{2}}\right) I_{m}(\xi)=0, \\
& I_{m}^{\prime}(\xi)+\frac{m}{\xi} I_{m}(\xi)=I_{m-1}(\xi), m \geq 1, \\
& I_{m}^{\prime}(\xi)-\frac{m}{\xi} I_{m}(\xi)=I_{m+1}(\xi), m \geq 0, \\
& I_{m-1}(\xi)-I_{m+1}(\xi)=\frac{2 m}{\xi} I_{m}(\xi), m \geq 1, \\
& I_{m}(\xi)=\sqrt{\frac{2}{\pi \xi}} e^{\xi}\left[1-\frac{4 m^{2}-1}{8 \xi}+O\left(|\xi|^{-2}\right)\right], \\
& |\arg (\xi)|<\frac{\pi}{2}-\delta,|\xi| \rightarrow+\infty .
\end{aligned}
$$

By (2.8) we have, for any complex number $s$,

$$
\frac{d}{d r}\left[\frac{I_{n+1 / 2}(r \sqrt{s+1})}{r^{1 / 2}}\right]=\frac{n}{r^{3 / 2}} I_{n+1 / 2}(r \sqrt{s+1})+\frac{\sqrt{s+1}}{r^{1 / 2}} I_{n+3 / 2}(r \sqrt{s+1}) .
$$

By differentiating (2.10) in $r$ and using (2.6) and (2.7), we obtain

$$
\begin{aligned}
\frac{d^{2}}{d r^{2}}\left[\frac{I_{n+1 / 2}(r \sqrt{s+1})}{r^{1 / 2}}\right]=\left(\frac{s+1}{r^{1 / 2}}+\right. & \left.\frac{n(n-1)}{r^{5 / 2}}\right) I_{n+1 / 2}(r \sqrt{s+1}) \\
& -\frac{2 \sqrt{s+1}}{r^{3 / 2}} I_{n+3 / 2}(r \sqrt{s+1}) .
\end{aligned}
$$

We obtain the equality (2.10) from [12, (3.23)], and (2.11) by differentiating (2.10) in $r$ and using (2.6) and (2.7).

We introduce the functions

$$
P_{n}(\xi)=\frac{I_{n+3 / 2}(\xi)}{\xi I_{n+1 / 2}(\xi)}, \quad n=0,1,2,3, \cdots
$$

Then ([12, Lemma 2.1]),

$$
\begin{aligned}
& P_{n}(\xi)=\frac{1}{\xi^{2} P_{n+1}(\xi)+(2 n+3)} \\
& P_{n}(0)=\frac{1}{2 n+3} \\
& \frac{d}{d \xi} P_{n}(\xi)=\frac{1}{\xi}-\frac{2 n+3}{\xi} P_{n}(\xi)-\xi P_{n}^{2}(\xi), \\
& \left|P_{n}(\xi)\right| \leq \sqrt{2} P_{n}(|\xi|) \leq \frac{\sqrt{2}}{2 n+3} \quad \text { for }|\arg (\xi)| \leq \pi / 4 .
\end{aligned}
$$


We also have, for any small $\delta>0$,

$$
\left|P_{n}(\xi)\right| \leq C(\delta) P_{n}(|\xi|) \quad \text { for }|\arg (\xi)| \leq \frac{\pi}{2}-\delta ;
$$

indeed, one can prove (2.17) by repeating the proof of (2.16) in [12, Lemma 2.1], noting that $\cos 2 \theta \geq-\cos 2 \delta>-1+\delta$ if $|\theta| \leq \frac{\pi}{2}-\delta$.

For real $r>0$,

$$
\begin{aligned}
& P_{n}(r)>P_{n+1}(r), n \geq 0, \\
& \frac{d}{d r} P_{n}(r)<0, \\
& \frac{2 n+5}{r^{2}+(2 n+3)(2 n+5)}<P_{n}(r) \\
& \quad \quad \frac{r^{2}+(2 n+5)(2 n+7)}{2(2 n+5) r^{2}+(2 n+3)(2 n+5)(2 n+7)}, \\
& \frac{1}{r}-\frac{n+1}{r^{2}}<P_{n}(r)<\frac{1}{r}-\frac{n+1}{r^{2}}+\frac{n^{2}+n+1}{2 r^{3}}, \\
& P_{n}(r)<\frac{1}{r} ;
\end{aligned}
$$

the inequalities (2.19)-(2.21) are taken from [12, Lemma 2.3] and the proof of (2.22) follows by comparison of $P_{n}(r)$ with $1 / r$, using the differential equation (2.15).

For simplicity of notation, we shall usually denote $R_{S}$ by $R$ throughout this paper. From (1.8) and [12, (2.11)] we have

$$
\frac{\widetilde{\sigma}}{3}=P_{0}(R) .
$$

Using the $P_{n}$ function we can write (1.10) in the form

$$
\mu_{n}(R)=n\left(\frac{n(n+1)}{2}-1\right) \frac{1}{R^{5} P_{0}(R)\left[P_{1}(R)-P_{n}(R)\right]} .
$$

\section{Lemma 2.1.}

$$
\begin{aligned}
& \lambda \equiv\left(\sigma_{S}\right)_{r}(R)=R P_{0}(R), \\
& \left.\frac{\partial^{2} \sigma_{S}}{\partial r^{2}}\right|_{r=R}=1-2 P_{0}(R), \\
& \left.\frac{\partial^{3} \sigma_{S}}{\partial r^{3}}\right|_{r=R}=R P_{0}(R)\left[1-2 P_{1}(R)\right],
\end{aligned}
$$

and

$$
\begin{aligned}
& \left.\frac{\partial p_{S}}{\partial r}\right|_{r=R}=0 \\
& \left.\frac{\partial^{2} p_{S}}{\partial r^{2}}\right|_{r=R}=-\mu\left[1-3 P_{0}(R)\right]=-\mu R^{2} P_{0}(R) P_{1}(R), \\
& \left.\frac{\partial^{3} p_{S}}{\partial r^{3}}\right|_{r=R}=-\mu R P_{0}(R)\left[1-2 P_{1}(R)\right] .
\end{aligned}
$$

Proof. The equality (2.25) follows directly by differentiating $\sigma_{S}(r)$ (in (1.7)) and using (2.3). Using (2.25) and the equation for $\sigma_{S}$, we get

$$
\left(\sigma_{S}\right)_{r r}(R)=-\frac{2}{R}\left(\sigma_{S}\right)_{r}(R)+\sigma_{S}(R)=1-2 P_{0}(R),
$$


which establishes (2.26). Differentiating the equation for $\sigma_{S}$ in $r$, we obtain

$$
\begin{aligned}
\left.\frac{\partial^{3} \sigma_{S}}{\partial r^{3}}\right|_{r=R} & =-\frac{2}{R}\left(\sigma_{S}\right)_{r r}(R)+\left(\frac{2}{R^{2}}+1\right)\left(\sigma_{S}\right)_{r}(R) \\
& =-\frac{2}{R}\left(1-2 P_{0}(R)\right)+\left(\frac{2}{R^{2}}+1\right) R P_{0}(R) \\
& =-\frac{2}{R}\left(1-3 P_{0}(R)\right)+R P_{0}(R) \\
& =R P_{0}(R)\left[1-2 P_{1}(R)\right] \quad(\text { by (2.13) with } n=0) .
\end{aligned}
$$

The equation (2.28) follows from the boundary condition of the stationary solution $p_{S}(r)$. Using (1.7), (2.23) and (2.26), we get

$$
\frac{\partial^{2} p_{S}(R)}{\partial r^{2}}=\mu\left(-\left(\sigma_{S}\right)_{r r}(R)+\frac{1}{3} \widetilde{\sigma}\right)=\mu\left[3 P_{0}(R)-1\right],
$$

which implies (2.29), by (2.13). Finally, (1.7) and (2.27) yield the relation (2.30).

We recall that the Laplace operator on a unit sphere has the form

$$
\Delta_{\omega} S(\theta, \phi)=\frac{1}{\sin \theta} \frac{\partial}{\partial \theta}\left(\sin \theta \frac{\partial S}{\partial \theta}\right)+\frac{1}{\sin ^{2} \theta} \frac{\partial^{2} S}{\partial \phi^{2}} \quad(\omega=(\theta, \phi)) .
$$

In particular,

$$
\Delta_{\omega} Y_{n, m}(\theta, \phi)=-n(n+1) Y_{n, m}(\theta, \phi) \quad(-n \leq m \leq n)
$$

where $Y_{n, m}$ is the spherical harmonic of order $(n, m)$, so that

$$
-\Delta\left(r^{n} Y_{n, m}\right)=0 .
$$

Using (2.5) we find that

$$
-\Delta\left(\frac{I_{n+1 / 2}(r)}{r^{1 / 2}} Y_{n, m}\right)+\left(\frac{I_{n+1 / 2}(r)}{r^{1 / 2}} Y_{n, m}\right)=0 .
$$

We recall that

$$
\begin{aligned}
& Y_{0,0}=\frac{1}{2 \sqrt{\pi}}, \\
& Y_{2,0}(\theta)=\frac{1}{4} \sqrt{\frac{5}{\pi}}\left(3 \cos ^{2} \theta-1\right), \\
& Y_{4,0}(\theta)=\frac{3}{16 \sqrt{\pi}}\left(35 \cos ^{4} \theta-30 \cos ^{2} \theta+3\right) .
\end{aligned}
$$

The following identities can be verified by direct computations:

$$
\begin{aligned}
& Y_{2,0}^{2}=\frac{1}{\sqrt{\pi}}\left[\frac{3}{7} Y_{4,0}+\frac{\sqrt{5}}{7} Y_{2,0}+\frac{1}{2} Y_{0,0}\right], \\
& \left(\frac{\partial Y_{2,0}}{\partial \theta}\right)^{2}=\frac{3}{\sqrt{\pi}}\left[-\frac{4}{7} Y_{4,0}+\frac{\sqrt{5}}{7} Y_{2,0}+Y_{0,0}\right] .
\end{aligned}
$$

Recall [17, page 305] that for any $n \geq 0$,

$$
Y_{n, 0}(\theta)=\sqrt{\frac{2 n+1}{4 \pi}} L_{n}(\cos \theta),
$$


where $L_{n}(x)$ are the Legendre polynomials:

$$
L_{n}(x)=\frac{1}{2^{n} n !} \frac{d^{n}}{d x^{n}}\left(x^{2}-1\right)^{n}, \quad L_{n}(-x)=(-1)^{n} L_{n}(x) .
$$

Clearly,

$$
\operatorname{span}\left\{L_{0}(x), L_{1}(x), L_{2}(x), \cdots, L_{n}(x)\right\}=\operatorname{span}\left\{1, x, x^{2}, \cdots, x^{n}\right\} .
$$

By (2.40) and 2.41), we see that $Y_{n, 0}(\theta)$ is an even function if $n$ is even and an odd function if $n$ is odd. It is well known ([17, page 306]) that, for any integer $n \geq 0$

$$
Y_{2,0} Y_{n, 0}=\sum_{j=0}^{\infty} c_{n j} Y_{j, 0} .
$$

Since $L_{n}(x)$ is a polynomial of degree $n, P_{2}(x) P_{n}(x)$ is a polynomial of degree $n+2$, so by (2.42) we must have

$$
c_{n j}=0 \quad \text { for } j \geq n+3 .
$$

Similarly, if we write

$$
\left(Y_{2,0}\right)_{\theta}\left(Y_{n, 0}\right)_{\theta}=\sum_{j=0}^{\infty} d_{n j} Y_{j, 0}
$$

then the above argument shows that $d_{n j}=0$ for $j \geq n+3$.

Lemma 2.2. There holds

$$
\begin{array}{ll}
c_{n j}=d_{n j}=0 & \text { if } j+n=o d d, \\
c_{n j}=d_{n j}=0 & \text { if }|n-j| \geq 3,
\end{array}
$$

and

$$
c_{j n}=c_{n j}, \quad d_{j n}+d_{n j}=6 c_{n j} \quad \text { for all } n, j ;
$$

furthermore,

$$
\sum_{j=0}^{n+2}\left(c_{n j}\right)^{2} \leq \frac{5}{4 \pi}
$$

and

$$
\sum_{j=0}^{n+2}\left(d_{n j}\right)^{2} \leq \frac{45}{16 \pi} n(n+1)
$$

Proof. If $n$ is even and $j$ is odd, then the product $Y_{2,0} Y_{2, n}$ is an even function while $Y_{j, 0}$ is odd, so that $c_{n j}=\left\langle Y_{2,0} Y_{n, 0}, Y_{j, 0}\right\rangle=0$. One can similarly prove (2.45) for all the remaining cases.

Clearly

$$
c_{n j}=\left\langle Y_{2,0} Y_{n, 0}, Y_{j, 0}\right\rangle=\left\langle Y_{2,0} Y_{j, 0}, Y_{n, 0}\right\rangle=c_{j n} .
$$

By symmetry, $c_{n j}=c_{j n}=0$ if $n \geq j+3$. 
For $d_{n j}$ we have

$$
\begin{aligned}
d_{n j}+d_{j n} & =\left\langle\left(Y_{2,0}\right)_{\theta}\left(Y_{n, 0}\right)_{\theta}, Y_{j, 0}\right\rangle+\left\langle\left(Y_{2,0}\right)_{\theta}\left(Y_{j, 0}\right)_{\theta}, Y_{n, 0}\right\rangle \\
& =2 \pi \int_{0}^{\pi}\left(Y_{n, 0} Y_{j, 0}\right)_{\theta}\left(Y_{2,0}\right)_{\theta} \sin \theta d \theta \\
& =-2 \pi \int_{0}^{\pi} Y_{n, 0} Y_{j, 0} \frac{\partial}{\partial \theta}\left[\left(Y_{2,0}\right)_{\theta} \sin \theta\right] d \theta \\
& =12 \pi \int_{0}^{\pi} Y_{n, 0} Y_{j, 0} Y_{2,0} \sin \theta d \theta
\end{aligned}
$$

by (2.31), (2.32), so that $d_{n j}+d_{j n}=6 c_{n j}$. It follows that if $n \geq j+3$, then $d_{n j}=-d_{j n}+6 c_{n j}=0$.

By (2.43) and the orthonormality of $Y_{n, m}$,

$$
\sum_{j=0}^{n+2}\left(c_{n j}\right)^{2}=2 \pi \int_{0}^{\pi}\left[Y_{2,0}(\theta) Y_{n, 0}(\theta)\right]^{2} \sin \theta d \theta \leq \max _{0 \leq \theta \leq \pi}\left|Y_{2,0}(\theta)\right|^{2} \leq \frac{5}{4 \pi} .
$$

From the equation $\Delta_{\omega} Y_{n, 0}+n(n+1) Y_{n, 0}=0$, we obtain, after multiplying by $Y_{n, 0} \sin \theta$ and integrating with respect to $\theta$,

$$
2 \pi \int_{0}^{\pi}\left(\frac{\partial Y_{n, 0}(\theta)}{\partial \theta}\right)^{2} \sin \theta d \theta=n(n+1) 2 \pi \int_{0}^{\pi}\left(Y_{n, 0}(\theta)\right)^{2} \sin \theta d \theta=n(n+1),
$$

so that by (2.43) and the orthonormality of $Y_{n, m}$,

$$
\sum_{j=0}^{n+2}\left(d_{n j}\right)^{2}=2 \pi \int_{0}^{\pi}\left[\frac{\partial Y_{2,0}}{\partial \theta} \frac{\partial Y_{n, 0}}{\partial \theta}\right]^{2} \sin \theta d \theta \leq n(n+1) \max _{0 \leq \theta \leq \pi}\left|\frac{\partial Y_{2,0}}{\partial \theta}\right|^{2},
$$

from which (2.48) follows.

Using (2.38), (2.39) we can compute the coefficients $c_{n j}$ and $d_{n j}$ for $n=2$ :

$$
\begin{array}{lll}
c_{20}=\frac{1}{2 \sqrt{\pi}}, & c_{22}=\frac{\sqrt{5}}{7 \sqrt{\pi}}, & c_{24}=\frac{3}{7 \sqrt{\pi}}, \\
d_{20}=\frac{3}{\sqrt{\pi}}, & d_{22}=\frac{3 \sqrt{5}}{7 \sqrt{\pi}}, & d_{24}=-\frac{12}{7 \sqrt{\pi}} .
\end{array}
$$

\section{The BIFURCATION PROBLEM}

In this section we give an alternative proof to [10] regarding the existence of bifurcation branches of symmetry-breaking solutions of the form (1.9). We want to use Theorem 1.1, and we therefore begin by defining a suitable function $F$.

Consider a family of domains with boundaries $\partial \Omega_{\varepsilon}: r=R_{S}+\widetilde{R}(\theta, \phi)$ where $\widetilde{R}(\theta, \phi)=\varepsilon S(\theta, \phi)$. Let $(\sigma, p)$ form the solution of

$$
\begin{aligned}
& -\Delta \sigma+\sigma=0 \quad \text { in } \Omega_{\varepsilon}, \\
& -\Delta p=\mu(\sigma-\widetilde{\sigma}) \quad \text { in } \Omega_{\varepsilon} \quad(\widetilde{\sigma}<1), \\
& \sigma=1 \quad \text { on } \partial \Omega_{\varepsilon}, \\
& p=\kappa \quad \text { on } \partial \Omega_{\varepsilon} .
\end{aligned}
$$


We define $F$ by

$$
F(\widetilde{R}, \mu)=\left.\frac{\partial p}{\partial n}\right|_{\partial \Omega_{\varepsilon}} .
$$

Then $\left(\sigma, p, R_{S}+\widetilde{R}\right)$ is a stationary solution of (1.1) - 1.5 if and only if $F(\widetilde{R}, \mu)=0$.

The function $S(\theta, \phi)$ may be viewed as a function defined on the unit sphere

$$
\Sigma=\{x:|x|=1\} .
$$

We shall later on assume that $S(\theta, \phi)$ is in $C^{m+\alpha}(\Sigma)$, that is, $S$ is $C^{m+\alpha}$ as a function defined on $\Sigma$; note that this does not mean that $S(\theta, \phi)$ is in $C^{m+\alpha}$ in the variable $(\theta, \phi)$.

As in [10] we introduce the Banach spaces

$$
\begin{aligned}
& X^{m+\alpha}=\left\{\widetilde{R} \in C^{m+\alpha}(\Sigma), \widetilde{R} \text { is } \pi \text {-periodic in } \theta, 2 \pi \text {-periodic in } \phi\right\}, \\
& X_{1}^{m+\alpha}=\text { closure of the linear space spanned by } \\
& \qquad\left\{Y_{j, 0}(\theta), j=0,1,2, \cdots\right\} \text { in } X^{m+\alpha}, \\
& X_{2}^{m+\alpha}=\text { closure of the linear space spanned by } \\
& \left\{Y_{j, 0}(\theta), j=0,2,4,6, \cdots\right\} \text { in } X^{m+\alpha} .
\end{aligned}
$$

We shall later on view $F(\widetilde{R}, \mu)$ as a mapping from $X^{m+3+\alpha}$ to $X^{m+\alpha}$ (or from $X_{j}^{m+3+\alpha}$ to $\left.X_{j}^{m+\alpha}\right)$.

As mentioned in Section 2, we shall usually denote $R_{S}$ simply by $R$.

In order to apply Theorem 1.1, we need to compute the Fréchet derivatives of $F$. To do that, we need the expansion of $(\sigma, p)$ of order $\varepsilon$. We can formally write, for any $\mu>0$,

$$
\begin{aligned}
& \sigma=\sigma_{S}+\varepsilon \sigma_{1}+O\left(\varepsilon^{2}\right), \\
& p=p_{S}+\varepsilon p_{1}+O\left(\varepsilon^{2}\right), \\
& \partial \Omega_{\varepsilon}: r=R+\varepsilon S(\theta, \phi) .
\end{aligned}
$$

Then, formally,

$$
\begin{aligned}
\left.\varepsilon \sigma_{1}\right|_{\partial B_{R}} & =\left.\varepsilon \sigma_{1}\right|_{\partial \Omega_{\varepsilon}}+O\left(\varepsilon^{2}\right) \\
& =\left[1-\sigma_{S}(R+\varepsilon S)\right]+O\left(\varepsilon^{2}\right)=-\varepsilon \lambda S+O\left(\varepsilon^{2}\right),
\end{aligned}
$$

where $\lambda=\left(\sigma_{S}\right)_{r}(R)$. Substituting (3.6) and (3.9) into (3.1) and (3.3) and dropping the $O\left(\varepsilon^{2}\right)$ terms, we find that $\sigma_{1}$ satisfies

$$
\begin{array}{ll}
-\Delta \sigma_{1}+\sigma_{1}=0 & \text { in } B_{R}, \\
\sigma_{1}=-\lambda S(\theta, \phi) & \text { on } \partial B_{R} .
\end{array}
$$

By [16],

$$
\kappa=\frac{1}{R}-\frac{\varepsilon}{R^{2}}\left(S+\frac{1}{2} \Delta_{\omega} S\right)+O\left(\varepsilon^{2}\right) ;
$$

in the subsequent Lemma 4.1 we shall prove a more refined formula for $\kappa$. We next expand the boundary condition for $p$. Since $\frac{\partial p_{S}(R)}{\partial r}=0$, the term $-\left(p_{S}\right)_{r}(R) S(\theta, \phi)$ vanishes. From (3.2), (3.4) we then obtain

$$
\begin{aligned}
& -\Delta p_{1}=\mu \sigma_{1} \quad \text { in } B_{R}, \\
& p_{1}=-\frac{1}{R^{2}}\left(S+\frac{1}{2} \Delta_{\omega} S\right) \quad \text { on } \partial B_{R} .
\end{aligned}
$$


If we write

$$
S(\theta, \phi)=\sum_{n=0}^{\infty} \sum_{m=-n}^{n} a_{n, m} Y_{n, m}(\theta, \phi),
$$

then we can solve $\left(\sigma_{1}, p_{1}\right)$ explicitly by

$$
\sigma_{1}(r, \theta, \phi)=-\sum_{n=0}^{\infty} \sum_{m=-n}^{n} \lambda a_{n, m} \frac{I_{n+1 / 2}(r)}{r^{1 / 2}} \frac{R^{1 / 2}}{I_{n+1 / 2}(R)} Y_{n, m}(\theta, \phi)
$$

and

$$
\begin{gathered}
p_{1}(r, \theta, \phi)=\sum_{n=0}^{\infty} \sum_{m=-n}^{n} a_{n, m} \frac{r^{n}}{R^{n}}\left[\frac{1}{R^{2}}\left(\frac{n(n+1)}{2}-1\right)-\mu \lambda\right] Y_{n, m}(\theta, \phi) \\
-\mu \sigma_{1}(r, \theta, \phi) .
\end{gathered}
$$

Indeed, this is easily verified using (2.33), (2.34).

Lemma 3.1. If $S \in C^{3+\alpha}(\Sigma)$ and $\sigma$ is a solution of (3.1) and (3.3), then

$$
\left\|\sigma-\sigma_{S}\right\|_{C^{3+\alpha}\left(\bar{\Omega}_{\varepsilon}\right)} \leq C|\varepsilon|\|S\|_{C^{3+\alpha}(\Sigma)},
$$

where the constant $C$ is independent of $\varepsilon$.

Proof. The function $\sigma_{S}(r)$ is well defined for all $r$, and it is clear that

$$
-\Delta\left(\sigma-\sigma_{S}\right)+\left(\sigma-\sigma_{S}\right)=0 \quad \text { in } \Omega_{\varepsilon} .
$$

On the boundary $\partial \Omega_{\varepsilon}=\{r=R+\varepsilon S(\theta, \phi)\}$, we have

$$
\begin{aligned}
\sigma(R+\varepsilon S, \theta, \phi)-\sigma_{S}(R+\varepsilon S) & =1-\sigma_{S}(R+\varepsilon S) \\
& =\sigma_{S}(R)-\sigma_{S}(R+\varepsilon S) .
\end{aligned}
$$

We can also differentiate this relation three times along $\partial \Omega_{\varepsilon}$, and thus we get

$$
\left\|\sigma-\sigma_{S}\right\|_{C^{3+\alpha}\left(\partial \Omega_{\varepsilon}\right)} \leq C|\varepsilon|\|S\|_{C^{3+\alpha}(\Sigma)} .
$$

Since $\partial \Omega_{\varepsilon}$ is in $C^{3+\alpha}$ uniformly in $\varepsilon$, we can use the Schauder estimates to obtain the estimates (3.18).

We next want to rigorously establish the equations (3.6), (3.7) by estimating the $O\left(\varepsilon^{2}\right)$ terms in the $C^{3+\alpha}$-norm. To do that we shall first transform $\Omega_{\varepsilon}$ onto the ball $B_{R}$ by the Hanzawa transformation.

The Hanzawa transformation is a diffeomorphism defined by

$$
(r, \theta, \phi)=H_{\varepsilon}\left(r^{\prime}, \theta^{\prime}, \phi^{\prime}\right) \equiv\left(r^{\prime}+\chi\left(R-r^{\prime}\right) \varepsilon S\left(\theta^{\prime}, \phi^{\prime}\right), \theta^{\prime}, \phi^{\prime}\right),
$$

where

$$
\chi \in C^{\infty}, \quad \chi(z)=\left\{\begin{array}{l}
0 \text { if }|z| \geq 3 \delta_{0} / 4 \\
1 \text { if }|z|<\delta_{0} / 4
\end{array}, \quad\left|\frac{d^{k} \chi}{d z^{k}}\right| \leq C / \delta_{0}^{k} .\right.
$$

Observe that $H_{\varepsilon}$ maps $B_{R}$ onto $\Omega_{\varepsilon}$ while keeping the ball $\left\{r<R-\left(3 \delta_{0} / 4\right)\right\}$ fixed. The inverse Hanzawa transformation $H_{\varepsilon}^{-1}$ maps $\Omega_{\varepsilon}$ onto $B_{R}$.

Let $\sigma_{1}$ be the solution of (3.10) - B.11). By the Schauder estimates $\sigma_{1} \in C^{3+\alpha}\left(\bar{B}_{R}\right)$ and $\left\|\sigma_{1}\right\|_{C^{3+\alpha}\left(\bar{B}_{R}\right)} \leq C\|S\|_{C^{3+\alpha}(\Sigma)}$. Setting

$$
\widetilde{\sigma}_{1}(r, \theta, \phi ; \varepsilon)=\sigma_{1}\left(H_{\varepsilon}^{-1}(r, \theta, \phi)\right) \quad \text { in } \Omega_{\varepsilon},
$$

we have

$$
\begin{array}{ll}
-\Delta \widetilde{\sigma}_{1}+\widetilde{\sigma}_{1}=\widetilde{f} & \text { in } \Omega_{\varepsilon}, \\
\widetilde{\sigma}_{1}=-\lambda S(\theta, \phi) & \text { on } \partial \Omega_{\varepsilon},
\end{array}
$$


where $\frac{1}{\varepsilon} \tilde{f}$ is a linear combination of products of at most second order derivatives of $\sigma_{1}$ and $S$ (see [10, Section 3]), and

$$
\|\widetilde{f}\|_{C^{1+\alpha}\left(\bar{\Omega}_{\varepsilon}\right)} \leq C|\varepsilon|\|S\|_{C^{3+\alpha}(\Sigma)} .
$$

Thus the function

$$
\psi=\sigma-\sigma_{S}-\varepsilon \widetilde{\sigma}_{1}
$$

is well defined in $\Omega_{\varepsilon}$ and satisfies

$$
\begin{aligned}
& -\Delta \psi+\psi=-\varepsilon \widetilde{f} \quad \text { in } \Omega_{\varepsilon}, \\
& \psi=-g \quad \text { on } \partial \Omega_{\varepsilon},
\end{aligned}
$$

where

$$
g(\theta, \phi)=\sigma_{S}(R+\varepsilon S(\theta, \phi))-1-\lambda S(\theta, \phi) .
$$

Similarly to (3.20), we have $\|g\|_{C^{3+\alpha}(\Sigma)} \leq C|\varepsilon|^{2}\|S\|_{C^{3+\alpha}(\Sigma)}$. Hence, the Schauder estimates yield the following result.

\section{Lemma 3.2.}

$$
\left\|\sigma-\sigma_{S}-\varepsilon \widetilde{\sigma}_{1}\right\|_{C^{3+\alpha}\left(\bar{\Omega}_{\varepsilon}\right)} \leq C|\varepsilon|^{2}\|S\|_{C^{3+\alpha}(\Sigma)} .
$$

We now turn to the solution $p$ of (3.2), (3.4). In view of Lemma 3.2

$$
-\Delta p=\mu\left(\sigma_{S}-\widetilde{\sigma}+\varepsilon \widetilde{\sigma}_{1}\right)+\varepsilon^{2} k \quad \text { in } \Omega_{\varepsilon},
$$

where

$$
\|k\|_{C^{3+\alpha}(\bar{\Omega})} \leq C\|S\|_{C^{3+\alpha}(\Sigma)} .
$$

We want to compute $\partial p / \partial n$ in terms of $S(\theta, \phi)$.

By the proof of (3.12) in [16,

$$
\left\|\kappa-\frac{1}{R}+\frac{\varepsilon}{R^{2}}\left(S+\frac{1}{2} \Delta_{\omega} S\right)\right\|_{C^{1+\alpha}(\Sigma)} \leq C|\varepsilon|^{2}\|S\|_{C^{3+\alpha}(\Sigma)} .
$$

Let $p_{1}$ be the solution of (3.13) and (3.14), and define

$$
\widetilde{p}_{1}(r, \theta, \phi ; \varepsilon)=p_{1}\left(H_{\varepsilon}^{-1}(r, \theta, \phi)\right) \quad \text { in } \Omega_{\varepsilon} .
$$

By the same procedure as before, using (3.31) we get

$$
\begin{aligned}
& \left\|p_{1}\right\|_{C^{1+\alpha}\left(\bar{B}_{R}\right)} \leq C\|S\|_{C^{3+\alpha}(\Sigma)}, \\
& \left\|p-p_{S}-\varepsilon \widetilde{p}_{1}\right\|_{C^{1+\alpha}\left(\partial \Omega_{\varepsilon}\right)} \leq C|\varepsilon|^{2}\|S\|_{C^{3+\alpha}(\Sigma)},
\end{aligned}
$$

and then, by (3.29), (3.30) and the Schauder estimates,

$$
\left\|p-p_{S}-\varepsilon \widetilde{p}_{1}\right\|_{C^{1+\alpha}\left(\bar{\Omega}_{\varepsilon}\right)} \leq C|\varepsilon|^{2}\|S\|_{C^{3+\alpha}(\Sigma)} .
$$

Since

$$
\frac{\partial p_{S}}{\partial r}(R)=0
$$

we have

$$
\left.\frac{\partial\left(p_{S}+\varepsilon \widetilde{p}_{1}\right)}{\partial n}\right|_{r=R+\varepsilon S(\theta, \phi)}=\varepsilon\left(\frac{\partial^{2} p_{S}(R)}{\partial r^{2}} S+\frac{\partial p_{1}(R, \theta, \phi)}{\partial r}\right)+O\left(|\varepsilon|^{2}\|S\|_{C^{3+\alpha}(\Sigma)}\right),
$$


and so, by (3.32),

$$
\left.\frac{\partial p}{\partial n}\right|_{r=R+\varepsilon S(\theta, \phi)}=\varepsilon\left(\frac{\partial^{2} p_{S}(R)}{\partial r^{2}} S+\frac{\partial p_{1}(R, \theta, \phi)}{\partial r}\right)+O\left(|\varepsilon|^{2}\|S\|_{C^{3+\alpha}(\Sigma)}\right) .
$$

The relation (3.34) shows that the mapping $(\widetilde{R}, \mu) \rightarrow F(\widetilde{R}, \mu)$ from $X^{m+3+\alpha}$ to $X^{m+\alpha}$ is bounded if $m=0$, and the same argument shows that the same is true for any $m \geq 0$. A similar argument shows that this mapping is Fréchet differentiable in $(\widetilde{R}, \mu)$; furthermore $\partial F(\widetilde{R}, \mu) / \partial \widetilde{R}(\partial F(\widetilde{R}, \mu) / \partial \mu)$ is obtained by solving a linearized problem about $(\widetilde{R}, \mu)$ with respect to $\widetilde{R}(\mu)$. By using the Schauder estimates we can then further obtain differentiability of $F(\widetilde{R}, \mu)$ to any order.

From (3.34) it follows that the Fréchet derivative of $F(\widetilde{R}, \mu)$ in $\widetilde{R}$, at $(0, \mu)$, is given by

$$
\left[F_{\widetilde{R}}(0, \mu)\right] S=\frac{\partial^{2} p_{S}(R)}{\partial r^{2}} S+\frac{\partial p_{1}(R, \theta, \phi)}{\partial r} .
$$

To compute the right-hand side, we note that $\frac{\partial^{2} p_{S}(R)}{\partial r^{2}}$ was already computed in (2.29), so that it remains to compute $\frac{\partial p_{1}(R, \theta, \phi)}{\partial r}$. We first compute $\partial \sigma_{1}(R, \theta, \phi) / \partial r$. By (3.16) we have

$$
\begin{aligned}
\left.\frac{\partial \sigma_{1}}{\partial r}\right|_{r=R} & =-\lambda \sum_{n=0}^{\infty} \sum_{m=-n}^{n} a_{n, m} \frac{\partial}{\partial r}\left[\frac{I_{n+1 / 2}(r)}{r^{1 / 2}} \frac{R^{1 / 2}}{I_{n+1 / 2}(R)}\right]_{r=R} Y_{n, m}(\theta, \phi) \\
& =-\lambda \sum_{n=0}^{\infty} \sum_{m=-n}^{n} a_{n, m}\left[\frac{n}{R}+\frac{I_{n+3 / 2}(R)}{I_{n+1 / 2}(R)}\right] Y_{n, m}(\theta, \phi) \text { by (2.10), }
\end{aligned}
$$

so that, by (3.17),

$$
\left.\frac{\partial p_{1}}{\partial r}\right|_{r=R}=\sum_{n=0}^{\infty} \sum_{m=-n}^{n} a_{n, m} \frac{n}{R}\left[\frac{1}{R^{2}}\left(\frac{n(n+1)}{2}-1\right)-\mu \lambda\right] Y_{n, m}(\theta, \phi)-\left.\mu \frac{\partial \sigma_{1}}{\partial r}\right|_{r=R} .
$$

Hence

$$
\left.\frac{\partial p_{1}}{\partial r}\right|_{r=R}=\sum_{n=0}^{\infty} \sum_{m=-n}^{n} a_{n, m}\left[\frac{n}{R^{3}}\left(\frac{n(n+1)}{2}-1\right)+\mu R^{2} P_{0}(R) P_{n}(R)\right] Y_{n, m} .
$$

Substituting (2.29) and (3.38) into (3.35) we get

$$
\begin{aligned}
{\left[F_{\widetilde{R}}(0, \mu)\right] S=} & \sum_{n=0}^{\infty} \sum_{m=-n}^{n} a_{n, m}\left[\frac{n}{R^{3}}\left(\frac{n(n+1)}{2}-1\right)\right. \\
& \left.+\mu R^{2} P_{0}(R) P_{n}(R)-\mu R^{2} P_{0}(R) P_{1}(R)\right] Y_{n, m}(\theta, \phi) ;
\end{aligned}
$$

in particular,

$$
\left[F_{\widetilde{R}}(0, \mu)\right] Y_{n, m}=\left[\frac{n}{R^{3}}\left(\frac{n(n+1)}{2}-1\right)-\mu R^{2} P_{0}(R)\left[P_{1}(R)-P_{n}(R)\right]\right] Y_{n, m}
$$

It follows that, for $n \neq 1,\left[F_{\widetilde{R}}(0, \mu)\right] Y_{n, m}=0$ if and only if $\mu=\mu_{n}(R)$, where $\mu_{n}(R)$ is defined by (2.24), or equivalently, by (1.10). Thus the operator $A_{\mu}=\left[F_{\widetilde{R}}(0, \mu)\right]$ 
maps $X_{1}^{3+\alpha}$ to $X_{1}^{\alpha}$, and

$$
\operatorname{ker} A_{\mu}=\left\{\begin{array}{l}
\left\{Y_{1,0}\right\} \text { if } \mu \neq \mu_{2}, \mu_{3}, \cdots, \\
\operatorname{span}\left\{Y_{n, 0}, Y_{1,0}\right\} \text { if } \mu=\mu_{n} .
\end{array}\right.
$$

If we work with the space $X_{1}^{3+\alpha}$, then $\operatorname{dim}\left(\operatorname{ker} A_{\mu_{n}}\right)=2$. In order to satisfy the requirement (ii) of Theorem 1.1 we shall henceforth work with the space $X_{2}^{3+\alpha}$. The operators are still well defined by the Algebra property of $X_{2}^{j+\alpha}$. In this $X_{2}^{3+\alpha}$ space, $\operatorname{dim}\left(\operatorname{ker} A_{\mu}\right)=1$ if $\mu=\mu_{n}$. If $n$ is even and $\geq 2$, then clearly the direct sum $\operatorname{Im} A_{\mu_{n}} \oplus\left\{Y_{n, 0}\right\}$ is the whole space $X_{2}^{\alpha}$ and therefore codim $\left(A_{\mu_{n}}\right)=1$. Differentiating (3.40) in $\mu$, we obtain

$$
\left[F_{\widetilde{R} \mu}\left(0, \mu_{n}\right)\right] S=\sum_{n=0}^{\infty} \sum_{m=-n}^{n} a_{n, m} R^{2} P_{0}(R)\left[P_{n}(R)-P_{1}(R)\right] Y_{n, m} .
$$

In particular,

$$
\left[F_{\widetilde{R} \mu}\left(0, \mu_{n}\right)\right] Y_{n, 0}=R^{2} P_{0}(R)\left[P_{n}(R)-P_{1}(R)\right] Y_{n, 0} \notin \operatorname{Im} A_{\mu_{n}} .
$$

We can now apply the Crandall-Rabinowitz theorem to conclude:

Theorem 3.3. The points $\left(0, \mu_{n}\right)$ ( $n$ even and $\geq 2$ ) are bifurcation points for the problem (3.1)-(3.4), and the corresponding free boundaries are of the form

$$
r=R+\varepsilon Y_{n, 0}(\theta)+o(\varepsilon) .
$$

In a similar way, we can consider $F(\widetilde{R}, \mu)$ as a mapping from $X_{2}^{m+3+\alpha}$ to $X_{2}^{m+\alpha}$ for any $m>1$ and then replace $o(\varepsilon)$ by an expansion to order $\varepsilon^{m}$ with error $o\left(\varepsilon^{m}\right)$.

Remark 3.1. Theorem 3.3 establishes bifurcation only for even $n \geq 2$. For any odd $n \geq 3$ we may apply the Crandall-Rabinowitz theorem in a more delicate manner, working with the space

$$
\begin{aligned}
& X=M^{k+\alpha}=\text { closure of the linear space spanned by } \\
& \qquad\left\{Y_{j, 0}(\theta), j=0,2,3,4,5,6, \cdots\right\} \text { in } X^{k+\alpha}
\end{aligned}
$$

for $k \geq 3$. Here we face the problem that $F$ does not map $M^{k+\alpha}$ into $M^{k-3+\alpha}$, but we could shift the center of the system in order to eliminate the mode of $Y_{1,0}$ and thus make the modified $F$ map $M^{k+\alpha}$ into $M^{k-3+\alpha}$. This shift is of higher order than $\varepsilon Y_{1,0}$, and its existence should follow by a fixed point theorem; cf. an analogous situation in Remark 1 in [10. The mapping $\widetilde{F}$ composed of $F$ followed by this shift of the origin will map $M^{k+\alpha}$ onto $M^{k-3+\alpha}$, and the Crandall-Rabinowitz theorem could then be applied to $\widetilde{F}$. The details of this approach would involve complicated computations and will not be pursued here.

For $n=2$, the bifurcation branch $(\widetilde{R}(\varepsilon), \mu(\varepsilon))$ satisfies $F(\widetilde{R}(\varepsilon), \mu(\varepsilon))=0$, $(\widetilde{R}(0), \mu(0))=\left(0, \mu_{2}\right)$, and

$$
\widetilde{R}(\varepsilon)=R+\varepsilon Y_{2,0}(\theta)+o(\varepsilon) .
$$

It will be important to compute $\mu^{\prime}(0)$. 


\section{The Computation of $\mu^{\prime}(0)$}

In order to compute $\mu^{\prime}(0)$, we need to derive, for each fixed $\mu$, the $\varepsilon^{2}$-order expansion in the special case $S=Y_{2,0}(\theta)$ :

$$
\begin{aligned}
& \sigma(r, \theta, \phi)=\sigma_{S}(r)+\varepsilon \sigma_{1}(r, \theta)+\varepsilon^{2} \sigma_{2}(r, \theta)+O\left(\varepsilon^{3}\right) \quad \text { in } \Omega_{\varepsilon}, \\
& p(r, \theta, \phi, \mu)=p_{S}(r, \mu)+\varepsilon p_{1}(r, \theta, \mu)+\varepsilon^{2} p_{2}(r, \theta, \mu)+O\left(\varepsilon^{3}\right) \quad \text { in } \Omega_{\varepsilon} .
\end{aligned}
$$

Lemma 4.1. If $\partial \Omega_{\varepsilon}: r=R+\varepsilon S(\theta, \phi)$, where $S \in C^{2}(\Sigma)$, then

$$
\left.\kappa\right|_{r=R+\varepsilon S(\theta, \phi)}=\frac{1}{R}-\frac{\varepsilon}{R^{2}}\left(S+\frac{1}{2} \Delta_{\omega} S\right)+\frac{\varepsilon^{2}}{R^{3}}\left(S^{2}+S \Delta_{\omega} S\right)+O\left(\varepsilon^{3}\right) .
$$

Proof. We use the notation $\vec{e}_{r}, \vec{e}_{\theta}, \vec{e}_{\phi}$ for the unit normal vectors in the $r, \theta, \phi$ directions, respectively. Then, written in the rectangular coordinates in $\mathbb{R}^{3}$,

$$
\begin{aligned}
& \vec{e}_{r}=(\sin \theta \cos \phi, \sin \theta \sin \phi, \cos \theta), \\
& \vec{e}_{\theta}=(\cos \theta \cos \phi, \cos \theta \sin \phi,-\sin \theta), \\
& \vec{e}_{\phi}=(-\sin \phi, \cos \phi, 0),
\end{aligned}
$$

and the gradient is given by

$$
\nabla_{x}=\vec{e}_{r} \partial_{r}+\vec{e}_{\theta} \frac{1}{r} \partial_{\theta}+\vec{e}_{\phi} \frac{1}{r \sin \theta} \partial_{\phi} \equiv \vec{e}_{r} \partial_{r}+\frac{1}{r} \nabla_{\omega} .
$$

If a surface is given by $r=R+\widetilde{R}(\theta, \phi)$, or, alternatively, by $\xi(r, \theta, \phi)=0$ where $\xi(r, \theta, \phi)=R+\widetilde{R}(\theta, \phi)-r$, then the normal vector is given by $\nabla_{x} \xi /\left|\nabla_{x} \xi\right|$, or

$$
\vec{n}=\frac{1}{\sqrt{1+\left|\nabla_{\omega} \widetilde{R}\right|^{2} /(R+\widetilde{R})^{2}}}\left(\vec{e}_{r}-\frac{\widetilde{R}_{\theta}}{R+\widetilde{R}} \vec{e}_{\theta}-\frac{\widetilde{R}_{\phi}}{(R+\widetilde{R}) \sin \theta} \vec{e}_{\phi}\right)
$$

and the mean curvature is then $-\left.\frac{1}{2} \operatorname{div} \frac{\nabla_{x} \xi}{\left|\nabla_{x} \xi\right|}\right|_{\xi=0}$, or

$$
\begin{aligned}
\left.\kappa\right|_{r=R+\widetilde{R}(\theta, \phi)} & =-\left.\frac{1}{2} \operatorname{div}\left(\frac{\nabla_{x}(\widetilde{R}-r)}{\left|\nabla_{x}(\widetilde{R}-r)\right|}\right)\right|_{r=R+\widetilde{R}(\theta, \phi)} \\
& =\frac{1}{2} \operatorname{div}\left[\frac{1}{\sqrt{1+\left|\nabla_{\omega} \widetilde{R}\right|^{2} / r^{2}}}\left(\vec{e}_{r}-\frac{\widetilde{R}_{\theta}}{r} \vec{e}_{\theta}-\frac{\widetilde{R}_{\phi}}{r \sin \theta} \vec{e}_{\phi}\right)\right]_{r=R+\widetilde{R}(\theta, \phi)}
\end{aligned}
$$

By direct computations,

$$
\begin{aligned}
& \operatorname{div} \vec{e}_{r}=\frac{1}{r} \vec{e}_{\theta} \cdot \partial_{\theta} \vec{e}_{r}+\frac{1}{r \sin \theta} \vec{e}_{\phi} \cdot \partial_{\phi} \vec{e}_{r}=\frac{2}{r}, \\
& \operatorname{div} \vec{e}_{\theta}=\frac{1}{r} \vec{e}_{\theta} \cdot \partial_{\theta} \vec{e}_{\theta}+\frac{1}{r \sin \theta} \vec{e}_{\phi} \cdot \partial_{\phi} \vec{e}_{\theta}=\frac{1}{r} \cot \theta, \\
& \operatorname{div} \vec{e}_{\phi}=\frac{1}{r} \vec{e}_{\theta} \cdot \partial_{\theta} \vec{e}_{\phi}+\frac{1}{r \sin \theta} \vec{e}_{\phi} \cdot \partial_{\phi} \vec{e}_{\phi}=0 .
\end{aligned}
$$


Using the formulas (2.31) and $\operatorname{div}(f \vec{m})=f \operatorname{div} \vec{m}+\nabla_{x} f \cdot \vec{m}$, we obtain

$$
\begin{aligned}
& \left.\kappa\right|_{r=R+\widetilde{R}(\theta, \phi)}=\left\{\frac{1}{\sqrt{1+\left|\nabla_{\omega} \widetilde{R}\right|^{2} / r^{2}}}\left(\frac{1}{r}-\frac{\cot \theta}{2 r^{2}} \widetilde{R}_{\theta}\right)\right. \\
& +\frac{1}{2}\left[\frac{\partial}{\partial r}\left(\frac{1}{\sqrt{1+\left|\nabla_{\omega} \widetilde{R}\right|^{2} / r^{2}}}\right)-\frac{1}{r} \frac{\partial}{\partial \theta}\left(\frac{1}{\sqrt{1+\left|\nabla_{\omega} \widetilde{R}\right|^{2} / r^{2}}} \frac{\widetilde{R}_{\theta}}{r}\right)\right. \\
& \left.\left.-\frac{1}{r \sin \theta} \frac{\partial}{\partial \phi}\left(\frac{1}{\sqrt{1+\left|\nabla_{\omega} \widetilde{R}\right|^{2} / r^{2}}} \frac{\widetilde{R}_{\phi}}{r \sin \theta}\right)\right]\right\}_{r=R+\widetilde{R}(\theta, \phi)} \\
& =\left\{\frac{1}{2} \frac{\partial}{\partial r}\left(\frac{1}{\sqrt{1+\left|\nabla_{\omega} \widetilde{R}\right|^{2} / r^{2}}}\right)+\frac{1}{r} \frac{1}{\sqrt{1+\left|\nabla_{\omega} \widetilde{R}\right|^{2} / r^{2}}}\right. \\
& -\frac{\widetilde{R}_{\theta}}{2 r^{2}} \frac{\partial}{\partial \theta}\left(\frac{1}{\sqrt{1+\left|\nabla_{\omega} \widetilde{R}\right|^{2} / r^{2}}}\right)-\frac{\widetilde{R}_{\phi}}{2 r^{2} \sin ^{2} \theta} \frac{\partial}{\partial \phi}\left(\frac{1}{\sqrt{1+\left|\nabla_{\omega} \widetilde{R}\right|^{2} / r^{2}}}\right) \\
& \left.-\frac{1}{2 r^{2} \sqrt{1+\left|\nabla_{\omega} \widetilde{R}\right|^{2} / r^{2}}} \Delta_{\omega} \widetilde{R}\right\}_{r=R+\widetilde{R}(\theta, \phi)}
\end{aligned}
$$

or

$$
\begin{aligned}
\left.\kappa\right|_{r=R+\widetilde{R}(\theta, \phi)}=\{ & \frac{1}{r}\left(1+\frac{\left|\nabla_{\omega} \widetilde{R}\right|^{2}}{r^{2}}\right)^{-3 / 2}\left[1+\frac{3}{2} \frac{\left|\nabla_{\omega} \widetilde{R}\right|^{2}}{r^{2}}\right] \\
& -\frac{\widetilde{R}_{\theta}}{2 r^{2}} \frac{\partial}{\partial \theta}\left(\frac{1}{\sqrt{1+\left|\nabla_{\omega} \widetilde{R}\right|^{2} / r^{2}}}\right) \\
& -\frac{\widetilde{R}_{\phi}}{2 r^{2} \sin ^{2} \theta} \frac{\partial}{\partial \phi}\left(\frac{1}{\sqrt{1+\left|\nabla_{\omega} \widetilde{R}\right|^{2} / r^{2}}}\right) \\
& \left.-\frac{1}{2 r^{2} \sqrt{1+\left|\nabla_{\omega} \widetilde{R}\right|^{2} / r^{2}}} \Delta_{\omega} \widetilde{R}\right\}_{r=R+\widetilde{R}(\theta, \phi)}
\end{aligned}
$$

Taking $\widetilde{R}=\varepsilon S(\theta, \phi)$ with small $|\varepsilon|$, we obtain

$$
\left.\kappa\right|_{r=R+\varepsilon S(\theta, \phi)}=\frac{1}{R+\varepsilon S}-\frac{\varepsilon}{2(R+\varepsilon S)^{2}} \Delta_{\omega} S+O\left(\varepsilon^{3}\right) .
$$

Since

$$
\begin{aligned}
& \frac{1}{R+\varepsilon S}=\frac{1}{R}-\frac{\varepsilon}{R^{2}} S+\frac{\varepsilon^{2}}{R^{3}} S^{2}+O\left(\varepsilon^{3}\right), \\
& \frac{\varepsilon}{2(R+\varepsilon S)^{2}}=\frac{\varepsilon}{2 R^{2}}-\frac{2 \varepsilon^{2}}{R^{3}} S+O\left(\varepsilon^{2}\right),
\end{aligned}
$$

(4.3) follows. 
Notice that for $\partial \Omega_{\varepsilon}: r=R+\varepsilon Y_{2,0}$, we have from (3.16), (3.17),

$$
\begin{aligned}
& \sigma_{1}(r, \theta)=-\lambda \frac{I_{5 / 2}(r)}{r^{1 / 2}} \frac{R^{1 / 2}}{I_{5 / 2}(R)} Y_{2,0}(\theta) \quad\left(\lambda=\left(\sigma_{S}\right)_{r}(R)\right), \\
& p_{1}(r, \theta, \mu)=\left\{\frac{r^{2}}{R^{2}}\left(\frac{2}{R^{2}}-\mu \lambda\right)+\mu \lambda \frac{I_{5 / 2}(r)}{r^{1 / 2}} \frac{R^{1 / 2}}{I_{5 / 2}(R)}\right\} Y_{2,0}(\theta) .
\end{aligned}
$$

From (4.1), (4.5) we formally derive for $\sigma_{2}$ the equations:

$$
\begin{aligned}
& -\Delta \sigma_{2}+\sigma_{2}=0 \quad \text { in } B_{R}, \\
& \sigma_{2}=-\frac{1}{2}\left(\sigma_{S}\right)_{r r}(R) Y_{2,0}^{2}(\theta)-\left(\sigma_{1}\right)_{r}(R, \theta) Y_{2,0}(\theta) \quad \text { on } \partial B_{R} .
\end{aligned}
$$

Taking $S=Y_{2,0}$ in (4.3) and using (2.32), we obtain the formula

$$
\left.\kappa\right|_{r=R+\varepsilon Y_{2,0}}=\frac{1}{R}+\varepsilon \frac{2}{R^{2}} Y_{2,0}-\varepsilon^{2} \frac{5}{R^{3}} Y_{2,0}^{2}+O\left(\varepsilon^{3}\right) .
$$

Using this expansion and (4.2), (4.6), we can also formally derive the equations for $p_{2}$ :

$$
\begin{aligned}
& -\Delta p_{2}=\mu \sigma_{2} \quad \text { in } B_{R} \\
& p_{2}=-\frac{5}{R^{3}} Y_{2,0}^{2}(\theta)-\frac{1}{2}\left(p_{S}\right)_{r r}(R) Y_{2,0}^{2}(\theta)-\left(p_{1}\right)_{r}(R, \theta) Y_{2,0}(\theta) \text { on } \partial B_{R} .
\end{aligned}
$$

We next prove a formula which will be important in the sequel.

\section{Lemma 4.2.}

$$
\begin{gathered}
\frac{1}{2} Y_{2,0} F_{\widetilde{R} \widetilde{R}}(0, \mu) Y_{2,0}=\left\{\left.\frac{\partial p_{2}}{\partial r}\right|_{r=R}+\left.Y_{2,0} \frac{\partial^{2} p_{1}}{\partial r^{2}}\right|_{r=R}+\left.\frac{1}{2}\left(Y_{2,0}\right)^{2} \frac{\partial^{3} p_{S}}{\partial r^{3}}\right|_{r=R}\right. \\
\left.-\left.\frac{1}{R^{2}} \frac{\partial Y_{2,0}}{\partial \theta} \frac{\partial p_{1}}{\partial \theta}\right|_{r=R}\right\} .
\end{gathered}
$$

Proof. Since the free boundary $r=R+\varepsilon Y_{2,0}(\theta)$ is independent of $\phi$, the function $p$ is also independent of $\phi$, and from (4.2) we get

$$
\begin{aligned}
\left.\frac{\partial p}{\partial r}\right|_{r=R+\varepsilon Y_{2,0}}= & \left.\frac{\partial p}{\partial r}\right|_{r=R}+\left.\varepsilon Y_{2,0} \frac{\partial^{2} p}{\partial r^{2}}\right|_{r=R}+\left.\frac{1}{2}\left(\varepsilon Y_{2,0}\right)^{2} \frac{\partial^{3} p}{\partial r^{3}}\right|_{r=R}+O\left(\varepsilon^{3}\right) \\
= & \varepsilon\left(\left.\frac{\partial p_{1}}{\partial r}\right|_{r=R}+\left.Y_{2,0} \frac{\partial^{2} p_{S}}{\partial r^{2}}\right|_{r=R}\right) \\
& +\varepsilon^{2}\left(\left.\frac{\partial p_{2}}{\partial r}\right|_{r=R}+\left.Y_{2,0} \frac{\partial^{2} p_{1}}{\partial r^{2}}\right|_{r=R}+\left.\frac{1}{2}\left(Y_{2,0}\right)^{2} \frac{\partial^{3} p_{S}}{\partial r^{3}}\right|_{r=R}\right)+O\left(\varepsilon^{3}\right)
\end{aligned}
$$

and

$$
\begin{aligned}
\left.\frac{\partial p}{\partial \theta}\right|_{r=R+\varepsilon Y_{2,0}} & =\left.\frac{\partial p}{\partial \theta}\right|_{r=R}+\left.\varepsilon Y_{2,0} \frac{\partial^{2} p}{\partial \theta \partial r}\right|_{r=R}+O\left(\varepsilon^{2}\right) \\
& =\left.\varepsilon \frac{\partial p_{1}}{\partial \theta}\right|_{r=R}+O\left(\varepsilon^{2}\right) .
\end{aligned}
$$


Using these results, we can compute

$$
\begin{aligned}
F\left(\varepsilon Y_{2,0}, \mu\right)=\left.\frac{\partial p}{\partial n}\right|_{r=R+\varepsilon Y_{2,0}}=\nabla p \cdot \vec{n} \\
=\quad\left(\vec{e}_{r} \frac{\partial p}{\partial r}+\vec{e}_{\theta} \frac{1}{r} \frac{\partial p}{\partial \theta}\right) \cdot\left(\vec{e}_{r}-\varepsilon \frac{1}{R} \frac{\partial Y_{2,0}}{\partial \theta} \vec{e}_{\theta}+O\left(\varepsilon^{2}\right)\right) \\
=\quad \varepsilon\left[\left.\frac{\partial p_{1}}{\partial r}\right|_{r=R}+\left.Y_{2,0} \frac{\partial^{2} p_{S}}{\partial r^{2}}\right|_{r=R}\right] \\
+\varepsilon^{2}\left\{\left.\frac{\partial p_{2}}{\partial r}\right|_{r=R}+\left.Y_{2,0} \frac{\partial^{2} p_{1}}{\partial r^{2}}\right|_{r=R}+\left.\frac{1}{2}\left(Y_{2,0}\right)^{2} \frac{\partial^{3} p_{S}}{\partial r^{3}}\right|_{r=R}\right. \\
\left.\quad-\left.\frac{1}{R^{2}} \frac{\partial Y_{2,0}}{\partial \theta} \frac{\partial p_{1}}{\partial \theta}\right|_{r=R}\right\}+O\left(\varepsilon^{3}\right) .
\end{aligned}
$$

On the other hand, since $F(0, \mu) \equiv 0$, Taylor's expansion gives

$$
F\left(\varepsilon Y_{2,0}, \mu\right)=\varepsilon F_{\widetilde{R}}(0, \mu) Y_{2,0}+\varepsilon^{2} \frac{1}{2} Y_{2,0} F_{\widetilde{R} \widetilde{R}}(0, \mu) Y_{2,0}+O\left(\varepsilon^{3}\right) .
$$

Comparing the $\varepsilon^{2}$ terms in this formula with the $\varepsilon^{2}$ terms in (4.13), we get the relation (4.12).

At this point we want to rigorously establish the $\varepsilon^{2}$-order expansions for $\sigma$ and $p$ in the special case of $\partial \Omega_{\varepsilon}: r=R+\varepsilon Y_{2,0}$.

Lemma 4.3. If

$$
\begin{aligned}
& -\Delta \sigma+\sigma=0 \quad \text { in } \Omega_{\varepsilon}, \\
& \sigma=1 \quad \text { on } \partial \Omega_{\varepsilon},
\end{aligned}
$$

where $\partial \Omega_{\varepsilon}: r=R+\varepsilon Y_{2,0}$, then

$$
\left\|\sigma-\left[\sigma_{S}+\varepsilon \sigma_{1}+\varepsilon^{2} \sigma_{2}\right]\right\|_{C^{3+\alpha}\left(\bar{\Omega}_{\varepsilon}\right)} \leq \text { const . }|\varepsilon|^{3},
$$

where $\sigma_{1}$ is the solution of

$$
\begin{array}{ll}
-\Delta \sigma_{1}+\sigma_{1}=0 & \text { in } \mathbb{R}^{3}, \\
\sigma_{1}=-\lambda Y_{2,0}(\theta) & \text { on } \partial B_{R},
\end{array}
$$

given by (4.5) and $\sigma_{2}$ is the solution of (4.7), (4.8), given by

$$
\begin{array}{r}
\sigma_{2}=\left[3 P_{0}(R)+R^{2} P_{0}(R) P_{2}(R)-\frac{1}{2}\right] \frac{1}{\sqrt{\pi}}\left[\frac{3}{7} \frac{I_{9 / 2}(r)}{r^{1 / 2}} \frac{R^{1 / 2}}{I_{9 / 2}(R)} Y_{4,0}(\theta)\right. \\
\left.+\frac{\sqrt{5}}{7} \frac{I_{5 / 2}(r)}{r^{1 / 2}} \frac{R^{1 / 2}}{I_{5 / 2}(R)} Y_{2,0}(\theta)+\frac{1}{2} \frac{I_{1 / 2}(r)}{r^{1 / 2}} \frac{R^{1 / 2}}{I_{1 / 2}(R)} Y_{0,0}\right] .
\end{array}
$$

Proof. Note that $\sigma_{S}, \sigma_{1}$ and $\sigma_{2}$ are defined (by their explicit formulas) for all $r>0$, and they satisfy the same elliptic equations $-\Delta u+u=0$ for all $r>0$. By (3.36) (with $a_{n, m}=1$ for $(n, m)=(2,0)$ and $a_{n, m}=0$ otherwise) and (2.25),

$$
\begin{aligned}
-\frac{\partial \sigma_{1}(R, \theta)}{\partial r} & =\lambda\left[\frac{2}{R}+R P_{2}(R)\right] Y_{2,0}(\theta) \\
& =\left[2 P_{0}(R)+R^{2} P_{0}(R) P_{2}(R)\right] Y_{2,0}(\theta) .
\end{aligned}
$$

Using also (2.26), we can rewrite the boundary condition (4.8) as

$$
\sigma_{2}=\left[3 P_{0}(R)+R^{2} P_{0}(R) P_{2}(R)-\frac{1}{2}\right] Y_{2,0}^{2}(\theta) \quad \text { on } \partial B_{R} .
$$


Recalling the formula (2.38) for $Y_{2,0}^{2}$, we can verify that $\sigma_{2}$ satisfies the boundary conditions (4.17) at $r=R$. Since $\sigma_{2}$ also satisfies the elliptic equation (4.7), we deduce, using (2.34), that $\sigma_{2}$ has the form (4.17).

The function

$$
\psi=\sigma(r, \theta, \phi)-\left\{\sigma_{S}(r)+\varepsilon \sigma_{1}+\varepsilon^{2} \sigma_{2}\right\}
$$

satisfies the differential equation

$$
-\Delta \psi+\psi=0 \quad \text { in } \Omega_{\varepsilon} .
$$

Since also $\|\psi\|_{C^{3+\alpha}(\Sigma)}=O\left(\varepsilon^{3}\right)$ on $\partial \Omega_{\varepsilon}$, the inequality (4.16) follows by the Schauder estimates.

Next we establish the second order expansion for $p$ :

Lemma 4.4. If

$$
\begin{aligned}
& -\Delta p=\mu(\sigma-\widetilde{\sigma}) \quad \text { in } \Omega_{\varepsilon}, \\
& p=\kappa \quad \text { on } \partial \Omega_{\varepsilon},
\end{aligned}
$$

where $\partial \Omega_{\varepsilon}: r=R+\varepsilon Y_{2,0}$, then

$$
\left\|p-\left[p_{S}+\varepsilon p_{1}+\varepsilon^{2} p_{2}(r, \theta, \phi)\right]\right\|_{C^{1+\alpha}\left(\bar{\Omega}_{\varepsilon}\right)} \leq \text { const . }|\varepsilon|^{3},
$$

where $p_{1}$ is given in (4.6), and $p_{2}$ is the solution of (4.10) (4.11), given by

$$
\begin{array}{r}
p_{2}=\left(-\frac{9}{R^{3}}+\frac{3}{2} \mu_{2} P_{0}(R)\right) \frac{1}{\sqrt{\pi}}\left[\frac{3}{7} \frac{r^{4}}{R^{4}} Y_{4,0}(\theta)\right. \\
\left.+\frac{\sqrt{5}}{7} \frac{r^{2}}{R^{2}} Y_{2,0}(\theta)+\frac{1}{2} Y_{0,0}\right]-\mu_{2} \sigma_{2} ;
\end{array}
$$

here $\sigma_{2}$ is given by (4.17).

Proof. The proof is similar to that of Lemma4.3. Using the second order expansion for $\sigma$ and the mean curvature $\kappa$, we find that $p_{2}$ satisfies (4.10)-4.11), where the first term of the boundary condition comes from the $\varepsilon^{2}$-terms in the expansion of curvature in $\kappa$, and the last two terms come from the deformation from $r=R$ to $r=R+\varepsilon Y_{2,0}$.

To solve for $p_{2}$, we observe that

$$
\Delta\left(p_{2}+\mu \sigma_{2}\right)=0 \quad \text { in } B_{R},
$$

and, by (4.11) and (4.8),

$$
\begin{aligned}
p_{2}+\mu \sigma_{2}= & -\left(\frac{5}{R^{3}}+\frac{1}{2}\left(p_{S}\right)_{r r}(R)+\frac{\mu}{2}\left(\sigma_{S}\right)_{r r}(R)\right) Y_{2,0}^{2}(\theta) \\
& -\left[\left(p_{1}\right)_{r}(R, \theta)+\mu\left(\sigma_{1}\right)_{r}(R, \theta)\right] Y_{2,0}(\theta) \quad \text { on } \partial B_{R} .
\end{aligned}
$$

From (2.26) and (2.29), we have

$$
\frac{1}{2}\left(p_{S}\right)_{r r}(R)+\frac{\mu}{2}\left(\sigma_{S}\right)_{r r}(R)=\frac{1}{2} \mu P_{0}(R),
$$

and from (3.37) (with $a_{n, m}=1$ if $(n, m)=(2,0)$ and $a_{n, m}=0$ otherwise) and (2.25),

$$
\begin{aligned}
\left(p_{1}\right)_{r}(R, \theta)+\mu\left(\sigma_{1}\right)_{r}(R, \theta) & =\frac{2}{R}\left[\frac{2}{R^{2}}-\mu \lambda\right] Y_{2,0}(\theta) \\
& =\left[\frac{4}{R^{3}}-2 \mu P_{0}(R)\right] Y_{2,0}(\theta)
\end{aligned}
$$


Thus we can rewrite the boundary condition for $p_{2}+\mu \sigma_{2}$ as

$$
p_{2}+\mu \sigma_{2}=\left(-\frac{9}{R^{3}}+\frac{3}{2} \mu P_{0}(R)\right) Y_{2,0}^{2}(\theta) \quad \text { on } \partial B_{R} .
$$

Using (2.38) and recalling (2.33), (2.34), we find that

$$
p_{2}+\mu \sigma_{2}=\left(-\frac{9}{R^{3}}+\frac{3}{2} \mu P_{0}(R)\right) \frac{1}{\sqrt{\pi}}\left[\frac{3}{7} \frac{r^{4}}{R^{4}} Y_{4,0}(\theta)+\frac{\sqrt{5}}{7} \frac{r^{2}}{R^{2}} Y_{2,0}(\theta)+\frac{1}{2} Y_{0,0}\right],
$$

and the assertion (4.24) then follows by recalling (4.17). Finally, the estimate (4.23) follows from the Schauder estimates.

Later on we shall need the following result.

Lemma 4.5. For $\mu=\mu_{2}$,

$$
\begin{aligned}
\left\langle\frac{1}{2} Y_{2,0} F_{\widetilde{R} \widetilde{R}}\left(0, \mu_{2}\right) Y_{2,0}, Y_{2,0}\right\rangle= & \frac{\sqrt{5}}{7 \sqrt{\pi}} \mu_{2} R P_{0}(R)\left\{-\frac{P_{1}(R)+P_{2}(R)}{2}\right. \\
& \left.-R^{2} P_{2}^{2}(R)+R^{2} P_{1}(R) P_{2}(R)\right\} .
\end{aligned}
$$

Proof. We start by computing the scalar product of $Y_{2,0}$ with each of the four terms on the right-hand side of (4.12). For the second term, we have, by (4.6), 2.11) with $n=2$, and (2.12),

$$
\left.Y_{2,0} \frac{\partial^{2} p_{1}}{\partial r^{2}}\right|_{r=R}=\left(\frac{4}{R^{4}}+\mu_{2} \lambda\left[1-2 P_{2}(R)\right]\right) Y_{2,0}^{2},
$$

so that, using (2.38) and $\left\langle Y_{n, 0}, Y_{m, 0}\right\rangle=\delta_{n}^{m}\left(\delta_{n}^{m}=1\right.$ if $n=m$ and $\delta_{n}^{m}=0$ if $\left.n \neq m\right)$, we obtain

$$
\left\langle\left. Y_{2,0} \frac{\partial^{2} p_{1}}{\partial r^{2}}\right|_{r=R}, Y_{2,0}\right\rangle=\left(\frac{4}{R^{4}}+\mu_{2} R P_{0}(R)\left[1-2 P_{2}(R)\right]\right) \frac{\sqrt{5}}{7 \sqrt{\pi}} .
$$

For the third term, we get, using (2.38) and (2.30),

$$
\left\langle\left.\frac{1}{2}\left(Y_{2,0}\right)^{2} \frac{\partial^{3} p_{S}}{\partial r^{3}}\right|_{r=R}, Y_{2,0}\right\rangle=\frac{\mu_{2}}{2} R P_{0}(R)\left[2 P_{1}(R)-1\right] \frac{\sqrt{5}}{7 \sqrt{\pi}} .
$$

For the fourth term in the braces, we obtain, since $p_{1}=\left(2 / R^{2}\right) Y_{2,0}(\theta)$ on $\partial B_{R}$,

$$
-\left.\frac{1}{R^{2}} \frac{\partial Y_{2,0}}{\partial \theta} \frac{\partial p_{1}}{\partial \theta}\right|_{r=R}=-\frac{2}{R^{4}}\left(\frac{\partial Y_{2,0}}{\partial \theta}\right)^{2} \quad \text { (by }(4.6) \text { ) , }
$$

and using (2.39) we find that

$$
\left\langle-\left.\frac{1}{R^{2}} \frac{\partial Y_{2,0}}{\partial \theta} \frac{\partial p_{1}}{\partial \theta}\right|_{r=R}, Y_{2,0}\right\rangle=-\frac{2}{R^{4}} \frac{3 \sqrt{5}}{7 \sqrt{\pi}} .
$$

The sum of the right-hand sides of (4.27), (4.28), and (4.30) is equal to

$$
\begin{aligned}
& =\frac{\sqrt{5}}{7 \sqrt{\pi}}\left\{-\frac{2}{R^{4}}+\mu_{2} R P_{0}(R)\left[\frac{1}{2}+P_{1}(R)-2 P_{2}(R)\right]\right\} \\
& =\frac{\mu_{2} \sqrt{5}}{7 \sqrt{\pi}} R P_{0}(R)\left\{-\frac{1}{2}\left[P_{1}(R)-P_{2}(R)\right]+\frac{1}{2}+P_{1}(R)-2 P_{2}(R)\right\} \\
& =\frac{\mu_{2} \sqrt{5}}{7 \sqrt{\pi}} R P_{0}(R)\left\{\frac{1}{2}+\frac{1}{2} P_{1}(R)-\frac{3}{2} P_{2}(R)\right\} .
\end{aligned}
$$


We finally compute the scalar product of $Y_{2,0}$ with the first term on the righthand side of (4.12). By (4.24),

$$
\left.\frac{\partial\left(p_{2}+\mu_{2} \sigma_{2}\right)}{\partial r}\right|_{r=R}=\left(-\frac{9}{R^{3}}+\frac{3}{2} \mu_{2} P_{0}(R)\right) \frac{1}{\sqrt{\pi}}\left[\frac{12}{7 R} Y_{4,0}(\theta)+\frac{2 \sqrt{5}}{7 R} Y_{2,0}(\theta)\right] .
$$

Since, by (4.17) and (2.10), (2.12),

$$
\begin{aligned}
\left.\mu_{2} \frac{\partial \sigma_{2}}{\partial r}\right|_{r=R}=\mu_{2} & {\left[3 P_{0}(R)+R^{2} P_{0}(R) P_{2}(R)-\frac{1}{2}\right] \frac{1}{\sqrt{\pi}}\left[\frac{3}{7}\left(\frac{4}{R}+R P_{4}(R)\right) Y_{4,0}(\theta)\right.} \\
+ & \left.\frac{\sqrt{5}}{7}\left(\frac{2}{R}+R P_{2}(R)\right) Y_{2,0}(\theta)+\frac{1}{2} R P_{0}(R) Y_{0,0}\right],
\end{aligned}
$$

we get

$$
\begin{aligned}
\left.\frac{\partial p_{2}}{\partial r}\right|_{r=R}= & \frac{\Lambda_{1}}{\sqrt{\pi}}\left[\frac{12}{7 R} Y_{4,0}(\theta)+\frac{2 \sqrt{5}}{7 R} Y_{2,0}(\theta)\right] \\
& +\frac{\Lambda_{2}}{\sqrt{\pi}}\left[\frac{3}{7} R P_{4}(R) Y_{4,0}(\theta)+\frac{\sqrt{5}}{7} R P_{2}(R) Y_{2,0}(\theta)+\frac{1}{2} R P_{0}(R) Y_{0,0}\right],
\end{aligned}
$$

where

$$
\begin{aligned}
& \Lambda_{1}=\left\{-\frac{9}{R^{3}}-\frac{3}{2} \mu_{2} P_{0}(R)-\mu_{2} R^{2} P_{0}(R) P_{2}(R)+\frac{\mu_{2}}{2}\right\}, \\
& \Lambda_{2}=-\mu_{2}\left\{3 P_{0}(R)+R^{2} P_{0}(R) P_{2}(R)-\frac{1}{2}\right\} .
\end{aligned}
$$

Hence

$$
\left\langle\frac{\partial p_{2}}{\partial r}(R, \theta), Y_{2,0}\right\rangle=\Lambda_{1} \frac{2 \sqrt{5}}{7 R \sqrt{\pi}}+\Lambda_{2} \frac{\sqrt{5}}{7 \sqrt{\pi}} R P_{2}(R) .
$$

Combining (4.34) with (4.31), the scalar product of $Y_{2,0}$ with the left-hand side of (4.12) takes the form:

$$
\begin{aligned}
& \left\langle\frac{1}{2} Y_{2,0} F_{\widetilde{R} \tilde{R}}\left(0, \mu_{2}\right) Y_{2,0}, Y_{2,0}\right\rangle \\
& =\frac{\sqrt{5}}{7 \sqrt{\pi}}\left\{\mu_{2} R P_{0}(R)\left[\frac{1}{2}+\frac{1}{2} P_{1}(R)-\frac{3}{2} P_{2}(R)\right]+\frac{2}{R} \Lambda_{1}+R P_{2}(R) \Lambda_{2}\right\} .
\end{aligned}
$$

We proceed to compute $\Lambda_{1}$ and $\Lambda_{2}$. Using (2.24), (2.13) with $n=0$, we obtain

$$
\begin{aligned}
\Lambda_{1} & =-\mu_{2} \frac{9}{4} R^{2} P_{0}(R)\left[P_{1}(R)-P_{2}(R)\right]-\mu_{2} R^{2} P_{0}(R) P_{2}(R)+\frac{\mu_{2}}{2}\left[1-3 P_{0}(R)\right] \\
& =\mu_{2} R^{2} P_{0}(R)\left(-\frac{9}{4}\left[P_{1}(R)-P_{2}(R)\right]-P_{2}(R)+\frac{1}{2} P_{1}(R)\right) \\
& =\mu_{2} R^{2} P_{0}(R)\left(-\frac{7}{4} P_{1}(R)+\frac{5}{4} P_{2}(R)\right),
\end{aligned}
$$

so that

$$
\frac{2}{R} \Lambda_{1}=\mu_{2} R P_{0}(R)\left(-\frac{7}{2} P_{1}(R)+\frac{5}{2} P_{2}(R)\right) .
$$

Similarly, using (2.13) with $n=0$, we get

$$
\Lambda_{2}=-\mu_{2}\left\{\frac{3}{2} P_{0}(R)+R^{2} P_{0}(R) P_{2}(R)-\frac{R^{2}}{2} P_{0}(R) P_{1}(R)\right\} .
$$


Thus, the right-hand side of (4.35) is equal to

$$
\begin{aligned}
& \frac{\sqrt{5}}{7 \sqrt{\pi}} \mu_{2} R P_{0}(R)\left\{\left[\frac{1}{2}+\frac{1}{2} P_{1}(R)-\frac{3}{2} P_{2}(R)\right]+\left(-\frac{7}{2} P_{1}(R)+\frac{5}{2} P_{2}(R)\right)\right. \\
& \left.\quad-\left\{\frac{3}{2} P_{2}(R)+R^{2} P_{2}^{2}(R)-\frac{R^{2}}{2} P_{2}(R) P_{1}(R)\right\}\right\} \\
& =\frac{\sqrt{5}}{7 \sqrt{\pi}} \mu_{2} R P_{0}(R)\left\{\frac{1}{2}-3 P_{1}(R)-\frac{1}{2} P_{2}(R)-R^{2} P_{2}^{2}(R)+\frac{1}{2} R^{2} P_{1}(R) P_{2}(R)\right\} \\
& =\frac{\sqrt{5}}{7 \sqrt{\pi}} \mu_{2} R P_{0}(R)\left\{-\frac{1}{2} P_{1}(R)-\frac{1}{2} P_{2}(R)-R^{2} P_{2}^{2}(R)+R^{2} P_{1}(R) P_{2}(R)\right\},
\end{aligned}
$$

where we used (2.13) with $n=1$ in deriving the last equality.

Lemma 4.6. For $R>0$,

$$
-\frac{1}{2} P_{1}(R)-\frac{1}{2} P_{2}(R)-R^{2} P_{2}^{2}(R)+R^{2} P_{1}(R) P_{2}(R)<0 .
$$

Proof. Using (2.15) we find that the function $f(r) \equiv r^{2}\left[P_{1}(r)-P_{2}(r)\right]-1$ satisfies

$$
\begin{aligned}
& \frac{d f(r)}{d r}+\left(\frac{3}{r}+r\left[P_{1}(r)+P_{2}(r)\right]\right) f(r) \\
& =2 r\left[P_{1}(r)-P_{2}(r)\right]+r^{2}\left\{-\frac{5}{r} P_{1}(r)+\frac{7}{r} P_{2}(r)-r\left[P_{1}^{2}(r)-P_{2}^{2}(r)\right]\right\} \\
& \quad+\left(\frac{3}{r}+r\left[P_{1}(r)+P_{2}(r)\right]\right) f(r) \\
& \quad-\frac{3}{r}-r\left[P_{1}(r)-P_{2}(r)\right]<-\frac{3}{r} \quad(\text { by }(2.18)) .
\end{aligned}
$$

Since clearly also

$$
f(r)<0 \quad \text { for small } r>0,
$$

it follows that $f(r)<0$ for all $0<r<\infty$. In particular,

$$
R^{2}\left[P_{1}(R)-P_{2}(R)\right]<1,
$$

so that

$$
2 R^{2} P_{2}(R)\left[P_{1}(R)-P_{2}(R)\right]<2 P_{2}(R)<P_{1}(R)+P_{2}(R),
$$

which implies (4.36).

We are now ready to establish the main theorem of this section:

Theorem 4.7. For the bifurcation branch $(\widetilde{R}(\varepsilon), \mu(\varepsilon))$ emanating from $\mu=\mu_{2}$,

$$
\begin{aligned}
\left.\frac{d \mu}{d \varepsilon}\right|_{\varepsilon=0}=\mu_{2}^{2} & \frac{\sqrt{5}}{28 \sqrt{\pi}} R^{4} P_{0}(R)\left\{-\frac{1}{2} P_{1}(R)-\frac{1}{2} P_{2}(R)\right. \\
& \left.-R^{2} P_{2}^{2}(R)+R^{2} P_{1}(R) P_{2}(R)\right\}<0 .
\end{aligned}
$$

Proof. Along the bifurcation branch emanating from $\mu=\mu_{2}$, we have $r=R+\widetilde{R}$, where

$$
\widetilde{R}=\varepsilon Y_{2,0}(\theta)+\varepsilon^{2} z_{2}(\theta)+O\left(\varepsilon^{3}\right)
$$

and $F(\widetilde{R}(\varepsilon), \mu(\varepsilon))=0$. 
By Taylor's expansion,

$$
0=F(0, \mu(\varepsilon))+F_{\widetilde{R}}(0, \mu(\varepsilon)) \widetilde{R}+\frac{1}{2} \widetilde{R} F_{\widetilde{R} \widetilde{R}}(0, \mu(\varepsilon)) \widetilde{R}+O\left(\varepsilon^{3}\right) .
$$

The first term of (4.40) on the right-hand side vanishes since $F(0, \mu)=0$ for any $\mu$. To compute the second term on the right-hand side, we write $\mu(\varepsilon)=\mu_{2}+\varepsilon \mu^{\prime}(0)+$ $O\left(\varepsilon^{2}\right)$, so that

$$
\begin{aligned}
F_{\widetilde{R}}(0, \mu(\varepsilon)) \widetilde{R} & =F_{\widetilde{R}}\left(0, \mu_{2}\right) \widetilde{R}+\varepsilon \mu^{\prime}(0) F_{\widetilde{R} \mu}\left(0, \mu_{2}\right) \widetilde{R}+O\left(\varepsilon^{3}\right) \\
& =\varepsilon F_{\widetilde{R}}\left(0, \mu_{2}\right) Y_{2,0}+\varepsilon^{2} F_{\widetilde{R}}\left(0, \mu_{2}\right) z_{2}+\varepsilon^{2} \mu^{\prime}(0) F_{\widetilde{R} \mu}\left(0, \mu_{2}\right) Y_{2,0}+O\left(\varepsilon^{3}\right) \\
& =\varepsilon^{2} F_{\widetilde{R}}\left(0, \mu_{2}\right) z_{2}+\varepsilon^{2} \mu^{\prime}(0) F_{\widetilde{R} \mu}\left(0, \mu_{2}\right) Y_{2,0}+O\left(\varepsilon^{3}\right),
\end{aligned}
$$

where we used the fact that $Y_{2,0} \in \operatorname{ker} F_{\widetilde{R}}\left(0, \mu_{2}\right)$.

The third term on the right-hand side of (4.40) is clearly equal to

$$
\varepsilon^{2} \frac{1}{2} Y_{2,0} F_{\widetilde{R} \widetilde{R}}\left(0, \mu_{2}\right) Y_{2,0}+O\left(\varepsilon^{3}\right) .
$$

Collecting $\varepsilon^{2}$-terms in (4.40), we obtain

$$
F_{\widetilde{R}}\left(0, \mu_{2}\right) z_{2}+\mu^{\prime}(0) F_{\widetilde{R} \mu}\left(0, \mu_{2}\right) Y_{2,0}+\frac{1}{2} Y_{2,0} F_{\widetilde{R} \widetilde{R}}\left(0, \mu_{2}\right) Y_{2,0}=0 .
$$

By (3.42) with $S=Y_{2,0}$ we further have

$$
F_{\widetilde{R} \mu}\left(0, \mu_{2}\right) Y_{2,0}=-R^{2} P_{0}(R)\left[P_{1}(R)-P_{2}(R)\right] Y_{2,0} .
$$

Note, by (3.39) (with $\mu=\mu_{2}$ so that the coefficient of $Y_{2,0}$ vanishes in (3.39)), $F_{\widetilde{R}}\left(0, \mu_{2}\right) z_{2}$ is orthogonal to $Y_{2,0}$. Hence if we take the scalar product of the lefthand side of (4.41) with $Y_{2,0}$ and use (4.42), we obtain

$$
\mu^{\prime}(0) R^{2} P_{0}(R)\left[P_{1}(R)-P_{2}(R)\right]=\left\langle\frac{1}{2} Y_{2,0} F_{\widetilde{R} \widetilde{R}}\left(0, \mu_{2}\right) Y_{2,0}, Y_{2,0}\right\rangle .
$$

Using (2.24) with $n=2$ and recalling (4.25), the assertion (4.39) easily follows.

\section{THE LINEARIZED PROBLEM}

We denote the stationary solution of the bifurcation branch emanating from $\mu_{2}$ by $(\sigma(\varepsilon), p(\varepsilon), \mu(\varepsilon), \partial \Omega(\varepsilon))$, so that

$$
\begin{aligned}
& \partial \Omega(\varepsilon): r=R_{S}+\widetilde{R}(\varepsilon)(\theta), \\
& \sigma(\varepsilon)(r, \theta)=\sigma_{S}(r)+\varepsilon \sigma_{1}(r, \theta)+O\left(\varepsilon^{2}\right), \\
& p(\varepsilon)(r, \theta)=p_{S}\left(r, \mu_{2}\right)+\varepsilon p_{1}(r, \theta)+O\left(\varepsilon^{2}\right),
\end{aligned}
$$

and

$$
\begin{aligned}
& \widetilde{R}(\varepsilon)(\theta)=\varepsilon Y_{2,0}(\theta)+O\left(\varepsilon^{2}\right), \\
& \mu(\varepsilon)=\mu_{2}+\mu^{\prime}(0) \varepsilon+O\left(\varepsilon^{2}\right),
\end{aligned}
$$

where $\mu^{\prime}(0)$ is given by (4.39). Note that since now $\mu=\mu(\varepsilon)$ is a function of $\varepsilon$, the $p_{1}$ here is different from the $p_{1}$ in Section 4 . For clarity we shall denote the $p_{1}$ from Section 4 by $p_{1}\left(r, \theta, \mu_{2}\right)$, so that the present $p_{1}$ is given by

$$
p_{1}(r, \theta)=p_{1}\left(r, \theta, \mu_{2}\right)+\mu^{\prime}(0) \frac{\partial p_{S}}{\partial \mu} .
$$


By (4.5), 4.6),

$$
\begin{aligned}
\sigma_{1}(r, \theta)= & -\lambda \frac{I_{5 / 2}(r)}{r^{1 / 2}} \frac{R^{1 / 2}}{I_{5 / 2}(R)} Y_{2,0}(\theta) \quad\left(\lambda=\left(\sigma_{S}\right)_{r}(R)\right), \\
p_{1}(r, \theta)= & p_{1}\left(r, \theta, \mu_{2}\right)+\mu^{\prime}(0) \frac{\partial p_{S}}{\partial \mu} \\
= & \left\{\frac{r^{2}}{R^{2}}\left(\frac{2}{R^{2}}-\mu_{2} \lambda\right)+\mu_{2} \lambda \frac{I_{5 / 2}(r)}{r^{1 / 2}} \frac{R^{1 / 2}}{I_{5 / 2}(R)}\right\} Y_{2,0}(\theta) \\
& +\mu^{\prime}(0)\left\{-\sigma_{S}(r)+\frac{1}{6} \widetilde{\sigma} r^{2}\right\} .
\end{aligned}
$$

We also have

$$
F(\widetilde{R}(\varepsilon), \mu(\varepsilon))=0 \text { for small }|\varepsilon| .
$$

We now fix $\varepsilon$, with $|\varepsilon|$ small. As in [12, Section 3], we can derive the linearized system near the stationary solution $(\sigma(\varepsilon), p(\varepsilon), \mu(\varepsilon), \partial \Omega(\varepsilon))$ by substituting

$$
\begin{aligned}
& \sigma(r, \theta, \phi, t)=\sigma(\varepsilon)(r, \theta)+\eta w(r, \theta, \phi, t), \\
& p(r, \theta, \phi, t)=p(\varepsilon)(r, \theta)+\eta q(r, \theta, \phi, t), \\
& \partial \Omega(\varepsilon): r=R+\widetilde{R}(\varepsilon)(\theta)+\eta \rho(\theta, \phi, t)
\end{aligned}
$$

into the system (1.1) - 1.6) and collecting the terms of order $\eta$. Before writting down the linearized system, let us examine the linearization of the boundary condition (1.5). It is easily seen that

$$
V_{n}=\eta\left(1+\frac{\left|\nabla_{\omega} \widetilde{R}\right|^{2}}{(R+\widetilde{R})^{2}}\right)^{-1 / 2} \rho_{t}+O\left(\eta^{2}\right)
$$

We also have

$$
\begin{aligned}
\left.\frac{\partial p}{\partial n}\right|_{r=R+\widetilde{R}+\eta \rho}= & \left.\eta \rho \frac{\partial}{\partial r} \nabla_{x} p(\varepsilon)(r, \theta) \cdot \vec{n}\right|_{r=R+\widetilde{R}}+\left.\eta \frac{\partial q}{\partial n}\right|_{r=R+\widetilde{R}} \\
& -\left.\eta\left(1+\frac{\left|\widetilde{R}_{\theta}\right|^{2}}{(R+\widetilde{R})^{2}}\right)^{-1 / 2} \frac{\rho_{\theta}}{(R+\widetilde{R})^{2}} \frac{\partial p(\varepsilon)}{\partial \theta}\right|_{r=R+\widetilde{R}}+O\left(\eta^{2}\right) .
\end{aligned}
$$

To prove (5.8) we compute

$$
\begin{aligned}
& \left(1+\frac{\left|\nabla_{\omega}(\widetilde{R}+\eta \rho)\right|^{2}}{(R+\widetilde{R}+\eta \rho)^{2}}\right)^{-1 / 2} \\
& =\left(1+\frac{\left|\nabla_{\omega} \widetilde{R}\right|^{2}}{(R+\widetilde{R})^{2}}+\frac{2 \eta \nabla_{\omega} \widetilde{R} \nabla_{\omega} \rho}{(R+\widetilde{R})^{2}}-\frac{2 \eta \rho\left|\nabla_{\omega} \widetilde{R}\right|^{2}}{(R+\widetilde{R})^{3}}+O\left(\eta^{2}\right)\right)^{-1 / 2} \\
& =\left(1+\frac{\left|\nabla_{\omega} \widetilde{R}\right|^{2}}{(R+\widetilde{R})^{2}}\right)^{-1 / 2} \\
& \quad-\eta\left(1+\frac{\left|\nabla_{\omega} \widetilde{R}\right|^{2}}{(R+\widetilde{R})^{2}}\right)^{-3 / 2}\left[\frac{\nabla_{\omega} \widetilde{R} \nabla_{\omega} \rho}{(R+\widetilde{R})^{2}}-\frac{\rho\left|\nabla_{\omega} \widetilde{R}\right|^{2}}{(R+\widetilde{R})^{3}}\right]+O\left(\eta^{2}\right) .
\end{aligned}
$$


Since $\widetilde{R}$ is independent of $\phi$,

$$
\begin{gathered}
\left.\vec{n}\right|_{r=R+\widetilde{R}+\eta \rho} \\
=\left(1+\frac{\left|\nabla_{\omega}(\widetilde{R}+\eta \rho)\right|^{2}}{(R+\widetilde{R}+\eta \rho)^{2}}\right)^{-1 / 2}\left[\vec{e}_{r}-\frac{\widetilde{R}_{\theta}+\eta \rho_{\theta}}{R+\widetilde{R}+\eta \rho} \vec{e}_{\theta}-\frac{\eta \rho_{\phi}}{(R+\widetilde{R}+\eta \rho) \sin \theta} \vec{e}_{\phi}\right] \\
=\left.\vec{n}\right|_{r=R+\widetilde{R}}-\eta\left(1+\frac{\left|\widetilde{R}_{\theta}\right|^{2}}{(R+\widetilde{R})^{2}}\right)^{-3 / 2} \\
\quad \times\left[\frac{\widetilde{R}_{\theta} \rho_{\theta}}{(R+\widetilde{R})^{2}}-\frac{\rho\left|\widetilde{R}_{\theta}\right|^{2}}{(R+\widetilde{R})^{3}}\right]\left(\vec{e}_{r}-\frac{\widetilde{R}_{\theta}}{R+\widetilde{R}} \vec{e}_{\theta}\right) \\
-\eta\left(1+\frac{\left|\widetilde{R}_{\theta}\right|^{2}}{(R+\widetilde{R})^{2}}\right)^{-1 / 2}\left(\frac{\rho_{\theta}}{R+\widetilde{R}} \vec{e}_{\theta}+\frac{\rho_{\phi}}{(R+\widetilde{R}) \sin \theta} \vec{e}_{\phi}\right)+O\left(\eta^{2}\right) .
\end{gathered}
$$

Next, from the equation

$$
\begin{aligned}
0 & =F(\widetilde{R}(\varepsilon), \mu(\varepsilon))=\left.\nabla_{x} p(\varepsilon)(r, \theta) \cdot \vec{n}\right|_{r=R+\widetilde{R}} \\
& =\left(1+\frac{\left|\widetilde{R}_{\theta}\right|^{2}}{(R+\widetilde{R})^{2}}\right)^{-1 / 2}\left[\frac{\partial}{\partial r} p(\varepsilon)(r, \theta)-\frac{\widetilde{R}_{\theta}}{(R+\widetilde{R})^{2}} \frac{\partial}{\partial \theta} p(\varepsilon)(r, \theta)\right]_{r=R+\widetilde{R}},
\end{aligned}
$$

we deduce that $\nabla_{x} p(\varepsilon)(r, \theta) \cdot\left(\vec{e}_{r}-\frac{\widetilde{R}_{\theta}}{(R+\widetilde{R})^{2}} \vec{e}_{\theta}\right)=0$ on $r=R+\widetilde{R}$, so that

$$
\begin{aligned}
& \left.\nabla_{x} p(\varepsilon)(r, \theta)\right|_{r=R+\widetilde{R}} \cdot\left(\left.\vec{n}\right|_{r=R+\widetilde{R}+\eta \rho}-\left.\vec{n}\right|_{r=R+\widetilde{R}}\right) \\
& =-\left.\eta\left(1+\frac{\left|\widetilde{R}_{\theta}\right|^{2}}{(R+\widetilde{R})^{2}}\right)^{-1 / 2} \frac{\rho_{\theta}}{R+\widetilde{R}} \frac{\partial p(\varepsilon)}{\partial \theta}\right|_{r=R+\widetilde{R}}+O\left(\eta^{2}\right) .
\end{aligned}
$$

From this expression for $\nabla_{x} p$ and the expression derived earlier for $\vec{n}$ we get

$$
\begin{aligned}
\left.\frac{\partial p}{\partial n}\right|_{r=R+\widetilde{R}+\eta \rho} & =\left.\nabla_{x}[p(\varepsilon)(r, \theta)+\eta q] \cdot \vec{n}\right|_{r=R+\widetilde{R}+\eta \rho} \\
& =\left.\nabla_{x} p(\varepsilon)(r, \theta)\right|_{r=R+\widetilde{R}}+\left.\eta \rho \frac{\partial}{\partial r} \nabla_{x} p(\varepsilon)(r, \theta)\right|_{r=R+\widetilde{R}},
\end{aligned}
$$

from which (5.8) follows.

The following system is the linearization of (1.1)-1.6) about $(\sigma(\varepsilon), p(\varepsilon), \Omega(\varepsilon))$ :

$$
\begin{aligned}
& w_{t}-\Delta w+w=0 \quad \text { in } \Omega(\varepsilon) \times\{t>0\}, \\
& w(R+\widetilde{R}(\varepsilon)(\theta), \theta, \phi, t)=-\left.\frac{\partial \sigma(\varepsilon)}{\partial r}\right|_{r=R+\widetilde{R}(\varepsilon)(\theta)} \rho, t>0, \\
& \Delta q=-\mu(\varepsilon) w \quad \text { in } \Omega(\varepsilon) \times\{t>0\}, \\
& q=\left.\frac{\partial \kappa}{\partial \widetilde{R}}\right|_{r=R+\widetilde{R}(\varepsilon)} \rho \quad \text { on } \partial \Omega(\varepsilon), t>0, \\
& \rho_{t}=-\left(1+\frac{\left(\widetilde{R}(\varepsilon)_{\theta}\right)^{2}}{(R+\widetilde{R}(\varepsilon))^{2}}\right)^{1 / 2}\left\{\left.\rho \frac{\partial}{\partial r} \nabla_{x} p(\varepsilon)(r, \theta) \cdot \vec{n}\right|_{r=R+\widetilde{R}(\varepsilon)}\right. \\
& \left.+\left.\frac{\partial q}{\partial n}\right|_{r=R+\widetilde{R}(\varepsilon)}\right\}+\left.\frac{\rho_{\theta}}{(R+\widetilde{R}(\varepsilon))^{2}} \frac{\partial}{\partial \theta} p(\varepsilon)\right|_{r=R+\widetilde{R}(\varepsilon)},
\end{aligned}
$$


where (5.14) follows from (5.7), (5.8) and $\left.\frac{\partial \kappa}{\partial \widetilde{R}}\right|_{r=R+\widetilde{R}} \rho$ is the Fréchet derivative of the curvature with respect to the boundary deformation in the direction $\rho$ at $r=R+\widetilde{R}$.

We next compute more explicitly the expressions $\frac{\partial \kappa}{\partial \widetilde{R}}, \frac{\partial}{\partial r} \nabla_{x} p \cdot \vec{n}$ and $\frac{\partial q}{\partial n}$ which appear in (15.14). To compute $\left.\frac{\partial \kappa}{\partial \widetilde{R}}\right|_{r=R+\widetilde{R}(\varepsilon)} \rho$, we use (14.3) with $\varepsilon S=\widetilde{R}(\varepsilon)+\eta \rho$ and $|\eta| \ll|\varepsilon|$ (notice also that $|\widetilde{R}(\varepsilon)| \leq$ const . $|\varepsilon|$ ) to obtain

$$
\begin{aligned}
& \left.\kappa\right|_{r=R+\widetilde{R}+\eta \rho}-\left.\kappa\right|_{r=R+\widetilde{R}} \\
& =-\frac{\eta}{R^{2}}\left(\rho+\frac{1}{2} \Delta_{\omega} \rho\right)+\frac{\eta}{R^{3}}\left(2 \widetilde{R} \rho+\widetilde{R} \Delta_{\omega} \rho+\rho \Delta_{\omega} \widetilde{R}\right)+O\left(\varepsilon^{2} \eta\right)+O\left(\eta^{2}\right),
\end{aligned}
$$

where $O\left(\varepsilon^{2} \eta\right)$ comes from the computation used to prove (4.3). Recalling that $\widetilde{R}=\varepsilon Y_{2,0}+O\left(\varepsilon^{2}\right)$, we get

$$
\left.\frac{\partial \kappa}{\partial \widetilde{R}}\right|_{r=R+\widetilde{R}(\varepsilon)} \rho=-\frac{1}{R^{2}}\left(\rho+\frac{1}{2} \Delta_{\omega} \rho\right)+\varepsilon \frac{Y_{2,0}}{R^{3}}\left(-4 \rho+\Delta_{\omega} \rho\right)+O\left(\varepsilon^{2}\right) .
$$

To compute $\frac{\partial}{\partial r} \nabla_{x} p \cdot \vec{n}$, note that

$$
\begin{aligned}
\left.\vec{n}\right|_{r=R+\widetilde{R}} & \left(1+\frac{\left|\left(\widetilde{R}_{\theta}\right)\right|^{2}}{(R+\widetilde{R})^{2}}\right)^{-1 / 2}\left[\vec{e}_{r}-\frac{\widetilde{R}_{\theta}}{R+\widetilde{R}} \vec{e}_{\theta}\right] \\
& =\vec{e}_{r}-\frac{\varepsilon}{R} \frac{\partial Y_{2,0}}{\partial \theta} \vec{e}_{\theta}+O\left(\varepsilon^{2}\right) .
\end{aligned}
$$

Hence

$$
\begin{aligned}
& \left.\frac{\partial}{\partial r} \nabla_{x} p(\varepsilon)(r, \theta) \cdot \vec{n}\right|_{r=R+\widetilde{R}(\varepsilon)} \\
= & \left(\left.\frac{\partial \nabla_{x} p(\varepsilon)}{\partial r}\right|_{r=R}+\left.\varepsilon Y_{2,0} \frac{\partial^{2} \nabla_{x} p(\varepsilon)}{\partial r^{2}}\right|_{r=R}\right) \cdot\left(\vec{e}_{r}-\frac{\varepsilon}{R} \frac{\partial Y_{2,0}}{\partial \theta} \vec{e}_{\theta}\right)+O\left(\varepsilon^{2}\right) \\
= & \frac{\partial^{2} p_{S}(R)}{\partial r^{2}}+\varepsilon\left(\frac{\partial^{2} p_{1}(R, \theta)}{\partial r^{2}}+\frac{\partial^{3} p_{S}(R)}{\partial r^{3}} Y_{2,0}\right)+O\left(\varepsilon^{2}\right) .
\end{aligned}
$$

Finally

$$
\begin{aligned}
& \left.\frac{\partial q}{\partial n}\right|_{r=R+\widetilde{R}(\varepsilon)}=\left.\nabla_{x} q \cdot \vec{n}\right|_{r=R+\widetilde{R}(\varepsilon)} \\
& \quad=\left(\left.\nabla_{x} q\right|_{r=R}+\left.\varepsilon Y_{2,0} \frac{\partial \nabla_{x} q}{\partial r}\right|_{r=R}\right) \cdot\left(\vec{e}_{r}-\frac{\varepsilon}{R} \frac{\partial Y_{2,0}}{\partial \theta} \vec{e}_{\theta}\right)+O\left(\varepsilon^{2}\right) \\
& \quad=\left.\frac{\partial q}{\partial r}\right|_{r=R}+\left.\varepsilon \frac{\partial^{2} q}{\partial r^{2}} Y_{2,0}\right|_{r=R}-\left.\frac{\varepsilon}{R^{2}} \frac{\partial Y_{2,0}}{\partial \theta} \frac{\partial q}{\partial \theta}\right|_{r=R}+O\left(\varepsilon^{2}\right) .
\end{aligned}
$$

We would like to establish linear stability or instability for the stationary solution $(\sigma(\varepsilon), p(\varepsilon), \partial \Omega(\varepsilon))$. The difficulty in doing so arises from the fact that this stationary solution has only been determined up to order $\varepsilon$. One could probably extend the solution as an analytic function in $\varepsilon$, as in [16, but it is difficult to compute the coefficients of $\varepsilon^{n}$ for large $n$. We therefore restrict ourselves to the system (5.10)(5.14) after we drop out all the $O\left(\varepsilon^{2}\right)$-terms, that is, to the system obtained from (5.10) (5.14) by keeping only the terms of orders $\varepsilon^{0}$ and $\varepsilon^{1}$. (Such an approximation 
was also done in 11] for a different free boundary problem.) Thus we wish to consider the system

$$
\begin{aligned}
& w_{t}-\Delta w+w=0 \quad \text { in } B_{R} \times\{t>0\}, \\
& {\left[w+\varepsilon Y_{2,0} \frac{\partial w}{\partial r}\right]_{r=R}=-\left[\frac{\partial \sigma_{S}}{\partial r}+\varepsilon \frac{\partial \sigma_{1}}{\partial r}+\varepsilon Y_{2,0} \frac{\partial^{2} \sigma_{S}}{\partial r^{2}}\right]_{r=R} \rho, t>0,} \\
& \Delta q=-\left[\mu_{2}+\varepsilon \mu^{\prime}(0)\right] w \quad \text { in } B_{R} \times\{t>0\}, \\
& {\left[q+\varepsilon Y_{2,0} \frac{\partial q}{\partial r}\right]_{r=R}=-\frac{1}{R^{2}}\left(\rho+\frac{1}{2} \Delta_{\omega} \rho\right)} \\
& +\varepsilon \frac{Y_{2,0}}{R^{3}}\left(-4 \rho+\Delta_{\omega} \rho\right) \quad \text { on } \partial B_{R}, t>0, \\
& \rho_{t}=-\left\{\frac{\partial^{2} p_{S}(R)}{\partial r^{2}}+\varepsilon\left(\frac{\partial^{2} p_{1}(R, \theta)}{\partial r^{2}}+\frac{\partial^{3} p_{S}(R)}{\partial r^{3}} Y_{2,0}\right)\right\} \rho \\
& -\left.\frac{\partial q}{\partial r}\right|_{r=R}-\left.\varepsilon Y_{2,0} \frac{\partial^{2} q}{\partial r^{2}}\right|_{r=R}+\left.\varepsilon \frac{1}{R^{2}} \frac{\partial Y_{2,0}}{\partial \theta} \frac{\partial q}{\partial \theta}\right|_{r=R}+\left.\varepsilon \frac{1}{R^{2}} \frac{\partial \rho}{\partial \theta} \frac{\partial p_{1}}{\partial \theta}\right|_{r=R} .
\end{aligned}
$$

We want to further simplify this system by breaking it into two coupled subsystems and dropping the $O\left(\varepsilon^{2}\right)$ terms that occur in this process. The first subsystem (for $\left(w_{1}, q_{1}\right)$ ) corresponds to the zero order terms in (5.19) subsystem (for $\left(w_{2}, q_{2}\right)$ ) corresponds to the $\varepsilon$-order terms, and

$$
\begin{aligned}
w & =w_{1}+\varepsilon w_{2}, \\
q & =q_{1}+\varepsilon q_{2} .
\end{aligned}
$$

The boundary conditions for $w_{1}$ and $w_{2}$ take the form

$$
\begin{gathered}
\left.w_{1}\right|_{r=R}=-\frac{\partial \sigma_{S}(R)}{\partial r} \rho \\
{\left[w_{2}+\varepsilon Y_{2,0} \frac{\partial w_{2}}{\partial r}\right]_{r=R}=-\left[\frac{\partial \sigma_{1}(R, \theta)}{\partial r}+\frac{\partial^{2} \sigma_{S}(R)}{\partial r^{2}} Y_{2,0}\right] \rho-\frac{\partial w_{1}(R, \theta, t)}{\partial r} Y_{2,0} .}
\end{gathered}
$$

Since $w=w_{1}+\varepsilon w_{2}, \varepsilon Y_{2,0} \frac{\partial w_{2}}{\partial r}$ will contribute an $O\left(\varepsilon^{2}\right)$ term to the solution $w$ and, as before, we drop such a term. Similarly we can obtain boundary conditions for $q_{1}, q_{2}$ and drop the $O\left(\varepsilon^{2}\right)$ terms from the boundary conditions; we also drop the $O\left(\varepsilon^{2}\right)$ terms from the differential equations for $q_{1}$ and $q_{2}$.

We then obtain the following system:

$$
\begin{aligned}
& \left(w_{1}\right)_{t}-\Delta w_{1}+w_{1}=0 \quad \text { in } B_{R} \times\{t>0\}, \\
& \left(w_{2}\right)_{t}-\Delta w_{2}+w_{2}=0 \quad \text { in } B_{R} \times\{t>0\}, \\
& w_{1}=-\frac{\partial \sigma_{S}(R)}{\partial r} \rho \quad \text { on } \partial B_{R} \times\{t>0\}, \\
& w_{2}=-\left[\frac{\partial \sigma_{1}(R, \theta)}{\partial r}+\frac{\partial^{2} \sigma_{S}(R)}{\partial r^{2}} Y_{2,0}\right] \rho-\frac{\partial w_{1}(R, \theta, t)}{\partial r} Y_{2,0} \\
& \quad \text { on } \partial B_{R} \times\{t>0\},
\end{aligned}
$$




$$
\begin{aligned}
\Delta q_{1}= & -\mu_{2} w_{1} \quad \text { in } B_{R} \times\{t>0\}, \\
\Delta q_{2}= & -\mu_{2} w_{2}-\mu^{\prime}(0) w_{1} \quad \text { in } B_{R} \times\{t>0\}, \\
q_{1}= & -\frac{1}{R^{2}}\left(\rho+\frac{1}{2} \Delta_{\omega} \rho\right) \quad \text { on } \partial B_{R} \times\{t>0\}, \\
q_{2}= & \frac{Y_{2,0}}{R^{3}}\left(-4 \rho+\Delta_{\omega} \rho\right)-\frac{\partial q_{1}(R, \theta, t)}{\partial r} Y_{2,0} \quad \text { on } \partial B_{R} \times\{t>0\}, \\
\rho_{t}=- & \left\{\frac{\partial^{2} p_{S}(R)}{\partial r^{2}}+\varepsilon\left(\frac{\partial^{2} p_{1}(R, \theta)}{\partial r^{2}}+\frac{\partial^{3} p_{S}(R)}{\partial r^{3}} Y_{2,0}\right)\right\} \rho \\
& -\left.\frac{\partial q_{1}}{\partial r}\right|_{r=R}-\varepsilon\left[\frac{\partial q_{2}}{\partial r}+\frac{\partial^{2} q_{1}}{\partial r^{2}} Y_{2,0}\right]_{r=R} \\
& +\left.\varepsilon \frac{1}{R^{2}} \frac{\partial Y_{2,0}}{\partial \theta} \frac{\partial q_{1}}{\partial \theta}\right|_{r=R}+\left.\varepsilon \frac{1}{R^{2}} \frac{\partial \rho}{\partial \theta} \frac{\partial p_{1}}{\partial \theta}\right|_{r=R} .
\end{aligned}
$$

This is the final form of the $\varepsilon$-order part of the linearized problem (5.10)-(5.14), and it is this system that we intend to consider for stability or instability. This system may also be viewed as the linearized problem of the original problem obtained by taking $\eta=\varepsilon^{2}$ and discarding all the $O(\eta)$ terms.

Until now we have taken the perturbation functions around the stationary solution to be functions of $(r, \theta, \phi)$. In the sequel we shall focus only on functions of $(r, \theta)$. The method we use can be extended to the perturbation by functions of $(r, \theta, \phi)$; however the computation becomes very messy and shall not be pursued here.

The system (5.24)-(5.32) is complemented with the initial conditions, which we shall take to be independent of $\phi$ :

$$
\begin{gathered}
\left.w_{1}\right|_{t=0}=w^{1,0}(r, \theta)=\sum_{n=0}^{\infty} w_{n, 0}^{1,0}(r) Y_{n, 0}(\theta), \\
\left.w_{2}\right|_{t=0}=0 \\
\left.\rho\right|_{t=0}=\rho_{0}(\theta)=\sum_{n=0}^{\infty} \rho_{n, 0}^{0} Y_{n, 0}(\theta)
\end{gathered}
$$

where $w_{n, 0}^{1,0}(r)$ and $\rho_{n, 0}^{0}$ are given. Then $w_{i}, q_{i}$ and $\rho$ are also independent of $\phi$, and, in particular,

$$
\rho=\sum_{n=0}^{\infty} \rho_{n, 0}(t) Y_{n, 0}(\theta)
$$

the $\rho_{n, 0}(t)$ are functions depending also on $\varepsilon$.

\section{The Laplace TRANSFORM OF THE Linearized PROBlem}

We shall denote the Laplace transform of a function $\Phi(t)$ by $\widehat{\Phi}(s)$, that is,

$$
\widehat{\Phi}(s)=\int_{0}^{\infty} e^{-s t} \Phi(t) d t .
$$

In order to prove stability or instability, for the linearized problem (5.24) we proceed as in [12] by first formally taking the Laplace transform of the system 
(5.24)-(5.32). We write

$$
\begin{aligned}
\rho & =\sum_{n=0}^{\infty} \rho_{n, 0}(t) Y_{n, 0}(\theta), \\
w_{j} & =\sum_{n=0}^{\infty} w_{n, 0}^{j}(r, t) Y_{n, 0}(\theta) \quad(j=1,2), \\
q_{j} & =\sum_{n=0}^{\infty} q_{n, 0}^{j}(r, t) Y_{n, 0}(\theta) \quad(j=1,2),
\end{aligned}
$$

and introduce the Laplace transforms:

$$
\begin{aligned}
\widehat{\rho} & =\sum_{n=0}^{\infty} \widehat{\rho}_{n, 0}(s) Y_{n, 0}(\theta), \\
\widehat{w}_{j} & =\sum_{n=0}^{\infty} \widehat{w}_{n, 0}^{j}(r, s) Y_{n, 0}(\theta) \quad(j=1,2), \\
\widehat{q}_{j} & =\sum_{n=0}^{\infty} \widehat{q}_{n, 0}^{j}(r, s) Y_{n, 0}(\theta) \quad(j=1,2) .
\end{aligned}
$$

Introducing the function

$$
\begin{gathered}
h_{n}(s) \equiv h_{n}\left(s, \mu_{2}, R\right)=\frac{1}{\mu_{2}}\left[\frac{s}{R^{2} P_{0}(R)}+\frac{n}{R^{5} P_{0}(R)}\left(\frac{n(n+1)}{2}-1\right)\right] \\
-P_{1}(R)+P_{n}(R \sqrt{s+1})
\end{gathered}
$$

as in [12, (3.26)], we shall prove that the $\widehat{\rho}_{n, 0}(s)$ satisfy a system of equations

$$
h_{n}(s) \widehat{\rho}_{n, 0}(s)+\varepsilon \sum_{j=n-2}^{n+2} B_{j, n}(s) \widehat{\rho}_{j, 0}(s)=F_{n}(s)+\varepsilon G_{n}(s),
$$

where, by convention, $B_{j, n}=0$ if either $j$ or $n$ is negative. We shall compute $B_{j, n}$ in terms of the coefficients of the system (5.24)-(5.32), and $F_{n}, G_{n}$ in terms of the initial data $w_{n, 0}^{1,0}, \rho_{n, 0}^{0}$. We perform the computation in four steps.

Step (i). We first evaluate the coefficients which involve the stationary solution. From (5.6),

$$
\frac{\partial^{2} p_{1}(R, \theta)}{\partial r^{2}}=\frac{\partial^{2} p_{1}\left(R, \theta, \mu_{2}\right)}{\partial r^{2}}+\mu^{\prime}(0)\left(-\frac{\partial^{2} \sigma_{S}(R)}{\partial r^{2}}+\frac{\widetilde{\sigma}}{3}\right)
$$

the first term on the right-hand side is computed by (4.26), and the second term by (2.26) and (2.23). Using also (2.13) with $n=0$, we obtain

$$
\frac{\partial^{2} p_{1}(R, \theta)}{\partial r^{2}}=\left(\frac{4}{R^{4}}+\mu_{2} \lambda\left[1-2 P_{2}(R)\right]\right) Y_{2,0}-\mu^{\prime}(0) R^{2} P_{0}(R) P_{1}(R) .
$$

From (4.18) and the boundary condition $p_{1}=\left(2 / R^{2}\right) Y_{2,0}(\theta)$ on $\partial B_{R}$ (see (4.6)), we obtain

$$
\begin{aligned}
& \frac{\partial \sigma_{1}(R, \theta)}{\partial r}=-\lambda\left(\frac{2}{R}+R P_{2}(R)\right) Y_{2,0} \\
& \frac{\partial p_{1}(R, \theta)}{\partial \theta}=\frac{2}{R^{2}} \frac{\partial Y_{2,0}}{\partial \theta}
\end{aligned}
$$


By (2.30), (6.9) and (2.24),

$$
\begin{aligned}
& \frac{\partial^{2} p_{1}(R, \theta)}{\partial r^{2}}+\frac{\partial^{3} p_{S}(R)}{\partial r^{3}} Y_{2,0} \\
& =\left(\frac{4}{R^{4}}+2 \mu_{2} R P_{0}(R)\left[P_{1}(R)-P_{2}(R)\right]\right) Y_{2,0}-\mu^{\prime}(0) R^{2} P_{0}(R) P_{1}(R) \\
& =\frac{12}{R^{4}} Y_{2,0}-\mu^{\prime}(0) R^{2} P_{0}(R) P_{1}(R) ;
\end{aligned}
$$

therefore, by Lemma 2.2

$$
\begin{aligned}
\left(\frac{\partial^{2} p_{1}(R, \theta)}{\partial r^{2}}+\frac{\partial^{3} p_{S}(R)}{\partial r^{3}} Y_{2,0}\right) \rho= & \frac{12}{R^{4}} \sum_{j=0}^{\infty}\left[\sum_{n=j-2}^{j+2} c_{n j} \rho_{n, 0}(t)\right] Y_{j, 0} \\
& -\mu^{\prime}(0) R^{2} P_{0}(R) P_{1}(R) \sum_{n=0}^{\infty} \rho_{n, 0}(t) Y_{n, 0} .
\end{aligned}
$$

This implies, after switching the indices $n$ and $j$, that

$$
\begin{aligned}
& \left\langle\left(\frac{\partial^{2} p_{1}(R, \theta)}{\partial r^{2}}+\frac{\partial^{3} p_{S}(R)}{\partial r^{3}} Y_{2,0}\right) \widehat{\rho}, Y_{n, 0}\right\rangle \\
& \quad=\frac{12}{R^{4}} \sum_{j=n-2}^{n+2} c_{j n} \widehat{\rho}_{j, 0}(s)-\mu^{\prime}(0) R^{2} P_{0}(R) P_{1}(R) \widehat{\rho}_{n, 0}(s) .
\end{aligned}
$$

Similarly, by (5.30),

$$
\begin{aligned}
\frac{\left(Y_{2,0}\right)_{\theta}}{R^{2}} \frac{\partial q_{1}(R, \theta)}{\partial \theta} & =\frac{1}{R^{4}} \sum_{n=0}^{\infty}\left(\frac{n(n+1)}{2}-1\right) \rho_{n, 0}(t)\left(Y_{n, 0}\right)_{\theta}\left(Y_{2,0}\right)_{\theta} \\
& =\frac{1}{R^{4}} \sum_{n=0}^{\infty}\left(\frac{n(n+1)}{2}-1\right) \rho_{n, 0}(t)\left(\sum_{j=0}^{n+2} d_{n j} Y_{j, 0}\right) \\
& =\frac{1}{R^{4}} \sum_{n=0}^{\infty}\left[\sum_{j=n-2}^{n+2}\left(\frac{j(j+1)}{2}-1\right) d_{j n} \rho_{j, 0}(t)\right] Y_{n, 0},
\end{aligned}
$$

so that

$$
\left\langle\frac{\left(Y_{2,0}\right)_{\theta}}{R^{2}} \frac{\partial q_{1}(R, \theta)}{\partial \theta}, Y_{n, 0}\right\rangle=\frac{1}{R^{4}} \sum_{j=n-2}^{n+2}\left(\frac{j(j+1)}{2}-1\right) d_{j n} \rho_{j, 0}(t) .
$$

Next, by (6.11),

$$
\begin{aligned}
\frac{\rho_{\theta}}{R^{2}} \frac{\partial p_{1}(R, \theta)}{\partial \theta} & =\frac{2}{R^{4}} \sum_{n=0}^{\infty} \rho_{n, 0}(t)\left(Y_{n, 0}\right)_{\theta}\left(Y_{2,0}\right)_{\theta} \\
& =\frac{2}{R^{4}} \sum_{n=0}^{\infty} \rho_{n, 0}(t)\left(\sum_{j=0}^{n+2} d_{n j} Y_{j, 0}\right) \\
& =\frac{2}{R^{4}} \sum_{n=0}^{\infty}\left[\sum_{j=n-2}^{n+2} d_{j n} \rho_{j, 0}(t)\right] Y_{n, 0}
\end{aligned}
$$

and therefore

$$
\left\langle\frac{\widehat{\rho}_{\theta}}{R^{2}} \frac{\partial p_{1}(R, \theta)}{\partial \theta}, Y_{n, 0}\right\rangle=\frac{2}{R^{4}} \sum_{j=n-2}^{n+2} d_{j n} \widehat{\rho}_{j, 0}(s)
$$


Step (ii). We next compute $\widehat{w}_{1}$ and $\widehat{q}_{1}$ in the same way as in [12. As in [12],

$$
\begin{gathered}
-\Delta \widehat{w}_{n, 0}^{1}+\left(\frac{n(n+1)}{r^{2}}+(s+1)\right) \widehat{w}_{n, 0}^{1}=w_{n, 0}^{1}(r, 0) \quad \text { in } B_{R}, \\
\widehat{w}_{n, 0}^{1}(R, s)=-\lambda \widehat{\rho}_{n, 0}(s),
\end{gathered}
$$

so that, by [12, (3.17)],

$$
\widehat{w}_{n, 0}^{1}=-\lambda \widehat{\rho}_{n, 0}(s) \frac{I_{n+1 / 2}(r \sqrt{s+1})}{r^{1 / 2}} \frac{R^{1 / 2}}{I_{n+1 / 2}(R \sqrt{s+1})}+\xi_{1 n}^{1},
$$

where $\xi_{1 n}^{1}$ satisfies

$$
\begin{gathered}
-\Delta \xi_{1 n}^{1}+\left(\frac{n(n+1)}{r^{2}}+(s+1)\right) \xi_{1 n}^{1}=w_{n, 0}^{1}(r, 0) \quad \text { in } B_{R}, \\
\xi_{1 n}^{1}(R, s)=0 .
\end{gathered}
$$

Also, by [12, (3.20)],

$$
\begin{aligned}
\widehat{q}_{n, 0}^{1}=\frac{r^{n}}{R^{n}} & {\left[\frac{1}{R^{2}}\left(\frac{n(n+1)}{2}-1\right)-\frac{\mu_{2} \lambda}{s+1}\right] \widehat{\rho}_{n, 0}-\frac{\mu_{2}}{s+1} \widehat{w}_{n, 0}+\psi_{1 n} } \\
=\left\{\frac{r^{n}}{R^{n}}\right. & {\left[\frac{1}{R^{2}}\left(\frac{n(n+1)}{2}-1\right)-\frac{\mu_{2} \lambda}{s+1}\right] } \\
& \left.+\frac{\mu_{2} \lambda}{s+1} \frac{I_{n+1 / 2}(r \sqrt{s+1})}{r^{1 / 2}} \frac{R^{1 / 2}}{I_{n+1 / 2}(R \sqrt{s+1})}\right\} \widehat{\rho}_{n, 0} \\
& -\frac{\mu_{2}}{s+1} \xi_{1 n}^{1}+\psi_{1 n}^{1},
\end{aligned}
$$

where $\psi_{1 n}^{1}$ is the solution of

$$
\begin{gathered}
-\Delta \psi_{1 n}^{1}+\frac{n(n+1)}{r^{2}} \psi_{1 n}^{1}=\frac{\mu_{2}}{s+1} w_{n, 0}^{1}(r, 0) \quad \text { in } B_{R}, \\
\left.\psi_{1 n}^{1}\right|_{r=R}=0 .
\end{gathered}
$$

Using (2.10), (2.11) and (2.12) in (6.19), we get

$$
\begin{aligned}
\left.\frac{\partial \widehat{q}_{n, 0}^{1}}{\partial r}\right|_{r=R}= & \left\{\frac{n}{R^{3}}\left(\frac{n(n+1)}{2}-1\right)+\mu_{2} \lambda R P_{n}(R \sqrt{s+1})\right\} \widehat{\rho}_{n, 0} \\
& +\frac{d}{d r}\left[-\frac{\mu_{2}}{s+1} \xi_{1 n}^{1}+\psi_{1 n}^{1}\right]_{r=R} \\
\left.\frac{\partial^{2} \widehat{q}_{n, 0}^{1}}{\partial r^{2}}\right|_{r=R}= & \left\{\frac{n(n-1)}{R^{4}}\left(\frac{n(n+1)}{2}-1\right)-2 \mu_{2} \lambda P_{n}(R \sqrt{s+1})\right. \\
& \left.+\mu_{2} \lambda\right\} \widehat{\rho}_{n, 0}+\frac{d^{2}}{d r^{2}}\left[-\frac{\mu_{2}}{s+1} \xi_{1 n}^{1}+\psi_{1 n}^{1}\right]_{r=R}
\end{aligned}
$$

Since

$$
\left.\frac{\partial^{2} \widehat{q}_{1}}{\partial r^{2}}\right|_{r=R} Y_{2,0}=\left.\sum_{n=0}^{\infty} \frac{\partial^{2} \widehat{q}_{n, 0}^{1}}{\partial r^{2}}\right|_{r=R} Y_{n, 0} Y_{2,0}=\sum_{n=0}^{\infty}\left[\left.\sum_{j=0}^{n+2} c_{n j} \frac{\partial^{2} \widehat{q}_{n, 0}^{1}}{\partial r^{2}}\right|_{r=R}\right] Y_{j, 0},
$$


(6.22) implies (after switching the indices $n$ and $j$ ) that

$$
\begin{aligned}
\left\langle\left.\frac{\partial^{2} \widehat{q}_{1}}{\partial r^{2}}\right|_{r=R} Y_{2,0}, Y_{n, 0}\right\rangle=\left.\sum_{j=n-2}^{n+2} c_{j n} \frac{\partial^{2} \widehat{q}_{j, 0}^{1}}{\partial r^{2}}\right|_{r=R} & \\
= & \sum_{j=n-2}^{n+2}\left\{\frac{j(j-1)}{R^{4}}\left(\frac{j(j+1)}{2}-1\right)-2 \mu_{2} \lambda P_{j}(R \sqrt{s+1})\right. \\
& \left.\quad+\mu_{2} \lambda\right\} c_{j n} \widehat{\rho}_{j, 0}+\sum_{j=n-2}^{n+2} c_{j n} \frac{d^{2}}{d r^{2}}\left[-\frac{\mu_{2}}{s+1} \xi_{1 j}^{1}+\psi_{1 j}^{1}\right]_{r=R} .
\end{aligned}
$$

Step (iii). The only remaining coefficient in (5.32) that is yet to be computed is $\partial q_{2}(R, \theta, t) / \partial r$.

We start with the computation of $w_{2}$. Since, by (2.26) and (6.10),

$$
\frac{\partial \sigma_{1}(R, \theta)}{\partial r}+\frac{\partial^{2} \sigma_{S}(R)}{\partial r^{2}} Y_{2,0}=\left[1-4 P_{0}(R)-R^{2} P_{0}(R) P_{2}(R)\right] Y_{2,0},
$$

the boundary condition (5.27) can be written as

$$
\begin{aligned}
& \left.w_{2}\right|_{r=R}=-\left[\frac{\partial \sigma_{1}(R, \theta)}{\partial r}+\frac{\partial^{2} \sigma_{S}(R)}{\partial r^{2}} Y_{2,0}\right]\left(\sum_{n=0}^{\infty} \rho_{n, 0}(t) Y_{n, 0}\right) \\
& -\left(\sum_{n=0}^{\infty} \frac{\partial w_{n, 0}^{1}(R, t)}{\partial r} Y_{n, 0}\right) Y_{2,0} \\
& =\sum_{n=0}^{\infty}\left\{-\left[1-4 P_{0}(R)-R^{2} P_{0}(R) P_{2}(R)\right] \rho_{n, 0}(t) Y_{n, 0}\right. \\
& \left.-\frac{\partial w_{n, 0}^{1}(R, t)}{\partial r} Y_{n, 0}\right\} Y_{2,0} .
\end{aligned}
$$

If we rewrite the product $Y_{2,0} Y_{n, 0}$ as a linear combination of $Y_{j, 0}(\theta)$, by (2.43), then we get

$$
\begin{gathered}
\left.w_{2}\right|_{r=R}=\sum_{j=0}^{\infty} \sum_{n} c_{n j}\left\{-\left[1-4 P_{0}(R)-R^{2} P_{0}(R) P_{2}(R)\right] \rho_{n, 0}(t)\right. \\
\left.-\frac{\partial w_{n, 0}^{1}(R, t)}{\partial r}\right\} Y_{j, 0} .
\end{gathered}
$$

Taking the Laplace transform and noting, by (6.17) and (2.10), that

$$
\left.\frac{\partial \widehat{w}_{n, 0}^{1}}{\partial r}\right|_{r=R}=-\lambda \widehat{\rho}_{n, 0}\left(\frac{n}{R}+(s+1) R P_{n}(R \sqrt{s+1})\right)+\left.\frac{d}{d r} \xi_{1 n}^{1}\right|_{r=R},
$$

we obtain

$$
\begin{aligned}
\left.\widehat{w}_{2}\right|_{r=R}=\sum_{j=0}^{\infty} & \sum_{n=j-2}^{j+2} c_{n j}\left\{-\left[1-4 P_{0}(R)-R^{2} P_{0}(R) P_{2}(R)\right] \widehat{\rho}_{n, 0}\right. \\
& \left.+\lambda \widehat{\rho}_{n, 0}\left(\frac{n}{R}+(s+1) R P_{n}(R \sqrt{s+1})\right)-\left.\frac{d}{d r} \xi_{1 n}^{1}\right|_{r=R}\right\} Y_{j, 0} .
\end{aligned}
$$


After switching the summation indices $j$ and $n$, we get

$$
\begin{aligned}
\widehat{w}_{n, 0}^{2}(R, s) & =\sum_{j=n-2}^{n+2} c_{j n}\left\{-\left[1-4 P_{0}(R)-R^{2} P_{0}(R) P_{2}(R)\right]\right. \\
& \left.+\lambda\left(\frac{j}{R}+(s+1) R P_{j}(R \sqrt{s+1})\right)\right\} \widehat{\rho}_{j, 0}-\left.\sum_{j=n-2}^{n+2} c_{j n} \frac{d}{d r} \xi_{1 j}^{1}\right|_{r=R} .
\end{aligned}
$$

Since $\widehat{w}^{2}$ is a solution of $s \widehat{w}^{2}-\Delta \widehat{w}^{2}+\widehat{w}^{2}=0$ (here we use (5.34)), its $n$-mode has the form

$$
\widehat{w}_{n, 0}^{2}(r, s)=\widehat{w}_{n, 0}^{2}(R, s) \frac{I_{n+1 / 2}(r \sqrt{s+1})}{r^{1 / 2}} \frac{R^{1 / 2}}{I_{n+1 / 2}(R \sqrt{s+1})},
$$

so that

$$
\frac{\partial \widehat{w}_{n, 0}^{2}(R, s)}{\partial r}=\widehat{w}_{n, 0}^{2}(R, s)\left(\frac{n}{R}+(s+1) R P_{n}(R \sqrt{s+1})\right) .
$$

We next compute the boundary condition for $\widehat{q}_{2}$. By (5.31) and (6.21),

$$
\begin{aligned}
\left.\widehat{q}_{2}\right|_{r=R}= & \left(\sum_{n=0}^{\infty} \frac{-4-n(n+1)}{R^{3}} \widehat{\rho}_{n, 0}(s) Y_{n, 0}\right) Y_{2,0}-\frac{\partial \widehat{q}_{1}(R, \theta, s)}{\partial r} Y_{2,0} \\
= & \left\{\sum _ { n = 0 } ^ { \infty } \left[-\frac{n}{R^{3}}\left(\frac{n(n+1)}{2}-1\right)-\mu_{2} \lambda R P_{n}(R \sqrt{s+1})\right.\right. \\
& \left.+\frac{-4-n(n+1)}{R^{3}}\right] \widehat{\rho}_{n, 0}(s) Y_{n, 0} \\
& \left.-\sum_{n=0}^{\infty} \frac{d}{d r}\left[\frac{-\mu_{2}}{s+1} \xi_{1 n}^{1}+\psi_{1 n}^{1}\right]_{r=R} Y_{n, 0}\right\} Y_{2,0} \\
= & \sum_{n=0}^{\infty} \sum_{j=0}^{n+2}\left\{\left[-\frac{n}{R^{3}}\left(\frac{n(n+1)}{2}-1\right)-\mu_{2} \lambda R P_{n}(R \sqrt{s+1})\right.\right. \\
& \left.\left.+\frac{-4-n(n+1)}{R^{3}}\right] \widehat{\rho}_{n, 0}(s) c_{n j}-\frac{d}{d r}\left[\frac{-\mu_{2}}{s+1} \xi_{1 n}^{1}+\psi_{1 n}^{1}\right]_{r=R} c_{n j}\right\} Y_{j, 0},
\end{aligned}
$$

so that (after switching $n$ and $j$ )

$$
\begin{aligned}
\widehat{q}_{n, 0}^{2}(R, s)= & \sum_{j=n-2}^{n+2}\left\{-\frac{j}{R^{3}}\left(\frac{j(j+1)}{2}-1\right)-\mu_{2} \lambda R P_{j}(R \sqrt{s+1})\right. \\
& \left.+\frac{-4-j(j+1)}{R^{3}}\right\} c_{j n} \widehat{\rho}_{j, 0}-\sum_{j=n-2}^{n+2} \frac{d}{d r}\left[\frac{-\mu_{2}}{s+1} \xi_{1 j}^{1}+\psi_{1 j}^{1}\right]_{r=R} c_{j n} .
\end{aligned}
$$

In order to compute $\widehat{q}_{n, 0}^{2}(r, s)$ we introduce the function $\psi=\widehat{q}_{n, 0}^{2}+\frac{\mu_{2}}{s+1} \widehat{w}_{n, 0}^{2}+$ $\frac{\mu^{\prime}(0)}{s+1} \widehat{w}_{n, 0}^{1}$. Since

$$
-\Delta \psi+\frac{n(n+1)}{r^{2}} \psi=\frac{\mu^{\prime}(0)}{s+1} w_{n, 0}^{1}(r, 0) \quad \text { in } B_{R},
$$

we can write

$$
\psi(r, s)=\frac{r^{n}}{R^{n}} \psi(R, s)+\psi_{1 n}^{2}
$$


where

$$
\begin{gathered}
-\Delta \psi_{1 n}^{2}+\frac{n(n+1)}{r^{2}} \psi_{1 n}^{2}=\frac{\mu^{\prime}(0)}{s+1} w_{n, 0}^{1}(r, 0) \quad \text { in } B_{R}, \\
\left.\psi_{1 n}^{2}\right|_{r=R}=0 .
\end{gathered}
$$

Hence

$$
\begin{gathered}
\widehat{q}_{n, 0}^{2}(r, s)=\frac{r^{n}}{R^{n}}\left(\widehat{q}_{n, 0}^{2}(R, s)+\frac{\mu_{2}}{s+1} \widehat{w}_{n, 0}^{2}(R, s)+\frac{\mu^{\prime}(0)}{s+1} \widehat{w}_{n, 0}^{1}(R, s)\right) \\
-\frac{\mu_{2}}{s+1} \widehat{w}_{n, 0}^{2}(r, s)-\frac{\mu^{\prime}(0)}{s+1} \widehat{w}_{n, 0}^{1}(r, s)+\psi_{1 n}^{2} .
\end{gathered}
$$

Using (6.25), (6.28) and (5.26) (according to which $\left.\widehat{w}_{n, 0}^{1}=-\lambda \widehat{\rho}_{n, 0}\right)$ we then get

$$
\begin{aligned}
\frac{\partial \widehat{q}_{n, 0}^{2}(R, s)}{\partial r} & =\frac{n}{R} \widehat{q}_{n, 0}^{2}(R, s)-\mu_{2} \widehat{w}_{n, 0}^{2}(R, s) R P_{n}(R \sqrt{s+1}) \\
& +\mu^{\prime}(0) \lambda \widehat{\rho}_{n, 0} R P_{n}(R \sqrt{s+1})+\frac{d}{d r}\left[\psi_{1 n}^{2}-\frac{\mu^{\prime}(0)}{s+1} \frac{d}{d r} \xi_{1 n}^{1}\right]_{r=R} .
\end{aligned}
$$

Substituting (6.26) and (6.30) into (6.34), we find that

$$
\frac{\partial \widehat{q}_{n, 0}^{2}(R, s)}{\partial r}=\sum_{j=n-2}^{n+2} k_{n, 0}^{2, j}(s) c_{j n} \widehat{\rho}_{j, 0}+\mu^{\prime}(0) \lambda \widehat{\rho}_{n, 0} R P_{n}(R \sqrt{s+1})+g_{n, 0}^{2}(s),
$$

where

$$
\begin{aligned}
k_{n, 0}^{2, j}(s)=\left\{\frac{n}{R}[\right. & -\frac{j}{R^{3}}\left(\frac{j(j+1)}{2}-1\right)-\mu_{2} \lambda R P_{j}(R \sqrt{s+1}) \\
& \left.+\frac{-4-j(j+1)}{R^{3}}\right] \\
& -\mu_{2} R P_{n}(R \sqrt{s+1})\left[-\left[1-4 P_{0}(R)-R^{2} P_{0}(R) P_{2}(R)\right]\right. \\
& \left.\left.+\lambda\left(\frac{j}{R}+(s+1) R P_{j}(R \sqrt{s+1})\right)\right]\right\}
\end{aligned}
$$

and

$$
\begin{aligned}
g_{n, 0}^{2}(s)=-\frac{n}{R} & \sum_{j=n-2}^{n+2} \frac{d}{d r}\left[\frac{-\mu_{2}}{s+1} \xi_{1 j}^{1}+\psi_{1 j}^{1}\right]_{r=R} c_{j n} \\
& +\left.\mu_{2} R P_{n}(R \sqrt{s+1}) \sum_{j=n-2}^{n+2} c_{j n} \frac{d}{d r} \xi_{1 j}^{1}\right|_{r=R} \\
& +\frac{d}{d r}\left[\psi_{1 n}^{2}-\frac{\mu^{\prime}(0)}{s+1} \frac{d}{d r} \xi_{1 n}^{1}\right]_{r=R} .
\end{aligned}
$$

It is clear that $g_{n, 0}^{2}(s)$ depends only on the initial data.

Step (iv). Taking the Laplace transform in (5.32) we get

$$
\begin{aligned}
0= & \left\{s \widehat{\rho}_{n, 0}(s)-\rho_{n, 0}(0)+\frac{\partial^{2} p_{S}(R)}{\partial r^{2}} \widehat{\rho}_{n, 0}+\left.\frac{\partial \widehat{q}_{n, 0}^{1}}{\partial r}\right|_{r=R}\right\} \\
& +\varepsilon\left\langle\left(\frac{\partial^{2} p_{1}(R, \theta)}{\partial r^{2}}+\frac{\partial^{3} p_{S}(R)}{\partial r^{3}} Y_{2,0}\right) \widehat{\rho}, Y_{n, 0}\right\rangle \\
& +\varepsilon\left\langle\frac{\partial \widehat{q}_{1}(R, \theta)}{\partial \theta} \frac{\left(Y_{2,0}\right)_{\theta}}{R^{2}}, Y_{n, 0}\right\rangle+\varepsilon\left\langle\frac{\partial p_{1}(R, \theta)}{\partial \theta} \frac{\widehat{\rho}_{\theta}}{R^{2}}, Y_{n, 0}\right\rangle \\
& +\varepsilon\left\langle\left.\frac{\partial^{2} \widehat{q}_{1}}{\partial r^{2}}\right|_{r=R} Y_{2,0}, Y_{n, 0}\right\rangle+\left.\varepsilon \frac{\partial \widehat{q}_{n, 0}^{2}}{\partial r}\right|_{r=R} .
\end{aligned}
$$


The last two terms in the braces were computed in (2.29) and (6.21); they are the same terms as in [12, (3.24)]. Recalling the definition of $h_{n}\left(s, \mu_{2}, R\right)$ in (6.7), the expression in braces in (6.38) takes the form

$$
\mu_{2} R^{2} P_{0}(R) h_{n}\left(s, \mu_{2}, R\right) \widehat{\rho}_{n, 0}-\rho_{n, 0}(0)-\frac{d}{d r}\left[\frac{\mu_{2}}{s+1} \xi_{1 n}^{1}-\psi_{1 n}^{1}\right]_{r=R} .
$$

The second term on the right-hand side of (6.38) was computed in (6.12), the third term in (6.13), the fourth term in (6.14), the fifth term in (6.23), and the sixth term in (6.35). Combining these results and comparing with (6.8), we obtain the following:

Lemma 6.1. The equation (6.8) holds with

$$
\begin{aligned}
\mu_{2} R^{2} P_{0}(R) B_{j, n}(s)= & \delta_{n}^{j} \mu^{\prime}(0) R^{2} P_{0}(R)\left[P_{n}(R \sqrt{s+1})-P_{1}(R)\right] \\
& +\left\{\frac{12}{R^{4}} c_{j n}+\frac{1}{R^{4}}\left(\frac{j(j+1)}{2}-1\right) d_{j n}\right. \\
& +\frac{2}{R^{4}} d_{j n}+k_{n, 0}^{2, j}(s) c_{j n}+\left[\frac{j(j-1)}{R^{4}}\left(\frac{j(j+1)}{2}-1\right)\right. \\
& \left.\left.-2 \mu_{2} \lambda P_{j}(R \sqrt{s+1})+\mu_{2} \lambda\right] c_{j n}\right\} \\
\mu_{2} R^{2} P_{0}(R) F_{n}(s)= & \rho_{n, 0}(0)+\frac{d}{d r}\left[\frac{\mu_{2}}{s+1} \xi_{1 n}^{1}-\psi_{1 n}^{1}\right]_{r=R}, \\
\mu_{2} R^{2} P_{0}(R) G_{n}(s)= & -g_{n, 0}^{2}(s)-\sum_{j=n-2}^{n+2} c_{j n} \frac{d^{2}}{d r^{2}}\left[-\frac{\mu_{2}}{s+1} \xi_{1 j}^{1}+\psi_{1 j}^{1}\right]_{r=R} .
\end{aligned}
$$

7. Estimates of $B_{2,2}(0), B_{n, j}(s), F_{n}(s)$ AND $G_{n}(s)$

\section{Lemma 7.1.}

$$
B_{2,2}(0)>0 \text {. }
$$

Proof. From (6.36) we have

$$
\begin{aligned}
k_{2,0}^{2,2}(0) & \\
=- & \frac{18}{R^{4}}-2 \mu_{2} R P_{0}(R) P_{2}(R) \\
& \quad+\mu_{2} R P_{2}(R)\left\{\left[1-4 P_{0}(R)-R^{2} P_{0}(R) P_{2}(R)\right]-\left[2 P_{0}(R)+R^{2} P_{0}(R) P_{2}(R)\right]\right\} \\
=- & \frac{9}{2} \mu_{2} R P_{0}(R)\left[P_{1}(R)-P_{2}(R)\right] \\
& \quad+\mu_{2} R P_{2}(R)\left[1-6 P_{0}(R)-2 R^{2} P_{0}(R) P_{2}(R)\right] \quad(\text { by (2.24) }) \\
=- & \frac{9}{2} \mu_{2} R P_{0}(R)\left[P_{1}(R)-P_{2}(R)\right] \\
& \quad+\mu_{2} R P_{2}(R)\left[R^{2} P_{0}(R) P_{1}(R)-3 P_{0}(R)-2 R^{2} P_{0}(R) P_{2}(R)\right] \quad(\text { by }(\underline{2.13})) \\
=\quad & \mu_{2} R P_{0}(R)\left\{-\frac{9}{2}\left[P_{1}(R)-P_{2}(R)\right]-3 P_{2}(R)+R^{2} P_{1}(R) P_{2}(R)-2 R^{2} P_{2}^{2}(R)\right\} .
\end{aligned}
$$


Since $d_{22}=3 c_{22}$,

$$
\begin{aligned}
& \mu_{2} R^{2} P_{0}(R) B_{2,2}(0) \\
& =\mu^{\prime}(0) R^{2} P_{0}(R)\left[P_{2}(R)-P_{1}(R)\right]+k_{2,0}^{2,2}(0) c_{22} \\
& \quad+\left\{\frac{28}{R^{4}}+\mu_{2} R P_{0}(R)\left[1-2 P_{2}(R)\right]\right\} c_{22} \\
& =\mu^{\prime}(0) R^{2} P_{0}(R)\left[P_{2}(R)-P_{1}(R)\right]+k_{2,0}^{2,2}(0) c_{22} \\
& \left.\quad+\mu_{2} R P_{0}(R)\left\{7\left[P_{1}(R)-P_{2}(R)\right]+1-2 P_{2}(R)\right\} c_{22} \quad \text { (by (2.24) }\right) \\
& =\mu^{\prime}(0) R^{2} P_{0}(R)\left[P_{2}(R)-P_{1}(R)\right]+\mu_{2} R P_{0}(R) c_{22}\left\{\frac{5}{2}\left[P_{1}(R)-P_{2}(R)\right]\right. \\
& \left.\quad+1-5 P_{2}(R)+R^{2} P_{1}(R) P_{2}(R)-2 R^{2} P_{2}^{2}(R)\right\} .
\end{aligned}
$$

Since $\mu^{\prime}(0)<0$ (by Theorem 4.7) and $P_{1}(R)>P_{2}(R)$ (by (2.18)), we have

$$
\mu^{\prime}(0) R^{2} P_{0}(R)\left[P_{2}(R)-P_{1}(R)\right]>0 .
$$

Hence if we prove that the quantity in the braces on the right-hand side of (7.2) is positive, then (7.1) will follow from (7.2). But this is indeed the case since, by (2.14), (2.18) and (2.19),

the quantity in the braces of (7.2)

$$
\begin{aligned}
& =1-5 P_{1}(R)+\frac{15}{2}\left[P_{1}(R)-P_{2}(R)\right]+R^{2} P_{1}(R) P_{2}(R)-2 R^{2} P_{2}^{2}(R) \\
& =\frac{15}{2}\left[P_{1}(R)-P_{2}(R)\right]+2 R^{2} P_{1}(R) P_{2}(R)-2 R^{2} P_{2}^{2}(R) \quad(\text { by }(\underline{2.13})) \\
& =\frac{15}{2}\left[P_{1}(R)-P_{2}(R)\right]+2 R^{2} P_{2}(R)\left[P_{1}(R)-P_{2}(R)\right]>0 .
\end{aligned}
$$

This completes the proof.

\section{Lemma 7.2.}

$$
B_{j, n}(s)=0 \quad \text { if } j+n=\text { odd. }
$$

Proof. The proof is immediate from (6.39) and (2.45).

From (7.4) it follows that odd and even terms in (6.8) actually decouple.

Lemma 7.3. If

$$
\begin{aligned}
& \left.\left.w_{1}\right|_{t=0} \equiv w_{2}\right|_{t=0} \equiv 0, \\
& \left.\rho_{n, 0}\right|_{t=0} \equiv 0 \quad \text { for } n \neq 2,\left.\quad \rho_{2,0}\right|_{t=0}=\mu_{2} R^{2} P_{0}(R),
\end{aligned}
$$

then (6.8) reduces to

$$
h_{n}(s) \widehat{\rho}_{n, 0}(s)=\delta_{n}^{2}-\varepsilon \sum_{j=n-2}^{n+2} B_{j, n}(s) \widehat{\rho}_{j, 0}(s), \quad n=0,2,4, \cdots,
$$

where $\widehat{\rho}_{n, 0}(s)=0$ for $n$ odd.

Proof. Under the assumptions (7.5), $\xi_{1 n}^{1}=\psi_{1 n}^{1}=\psi_{1 n}^{2}=0$ and $g_{n, 2}=0$, so that, $G_{n}(s)=0$ by (6.41) and $F_{n}(s)=\delta_{n}^{2}$ by (6.40). 


\section{Lemma 7.4.}

$$
\left|B_{j, n}(s)\right| \leq C(n+1)^{3} \quad \text { for } \operatorname{Re} s>-\delta_{1},
$$

for some positive constant $\delta_{1}$.

Proof. By Lemma 2.2, $\left|c_{n j}\right| \leq 1$ and $\left|d_{n j}\right| \leq n+1$, and $B_{j, n}(s)=0$ if $|j-n| \geq 3$ or if $j+n=$ odd. From (6.36) we see that the factor $(s+1)$ in $k_{n, 0}^{2, j}$ appears only in $(s+1) P_{n}(R \sqrt{s+1}) P_{j}(R \sqrt{s+1})$ and is therefore also bounded, by (2.16) and (2.22).

Hence

$$
\left|k_{n, 0}^{2, j}(s)+\frac{n}{R^{4}} \frac{j^{2}(j+1)}{2}\right| \leq C(n+1)(j+1)^{2} .
$$

From 6.39) we also have

$$
\left|\mu_{2} R^{2} P_{0}(R) B_{j, n}(s)-\left(k_{n, 0}^{2, j}(s)+\frac{j(j-1)}{R^{4}} \frac{j(j+1)}{2}\right) c_{j n}\right| \leq C(j+1)^{3} .
$$

If $c_{j n} \neq 0$, then $|n-j| \leq 2$ so that

$$
\left|\left(-\frac{n}{R^{4}} \frac{j^{2}(j+1)}{2}+\frac{j(j-1)}{R^{4}} \frac{j(j+1)}{2}\right) c_{j n}\right|=\frac{j^{2}(j+1)|j-n+1|}{R^{4}} \leq \frac{C(n+1)^{3}}{R^{4}},
$$

and combining this with the preceding two estimates, (7.7) follows.

For $n \neq 2$, we can divide the equation (6.8) by $h_{n}$ and take the inverse Laplace transform in (6.8) to obtain

$$
\rho_{n, 0}(t)+\varepsilon \sum_{j=n-2}^{n+2}\left(b_{j, n} * \rho_{j, 0}\right)(t)=f_{n}(t),
$$

where $b_{j, n}(t)$ is the inverse Laplace transform of $B_{j, n}(s) / h_{n}(s)$ and $f_{n}(t)$ is the inverse Laplace transform of $\left\{F_{n}(s)+\varepsilon G_{n}(s)\right\} / h_{n}(s)$.

For $n=2$, we write (6.8) as

$$
\left\{h_{2}(s)+\varepsilon B_{2,2}(s)\right\} \widehat{\rho}_{2,0}(s)+\varepsilon\left\{B_{0,2}(s) \widehat{\rho}_{0,0}(s)+B_{4,2}(s) \widehat{\rho}_{4,0}(s)\right\}=F_{2}(s)+\varepsilon G_{2}(s) .
$$

If we divide by $h_{2}(s)+\varepsilon B_{2,2}(s)$ and then take the inverse Laplace transform, we obtain

$$
\rho_{2,0}(t)+\varepsilon\left[\left(b_{0,2} * \rho_{0,0}\right)(t)+\left(b_{4,2} * \rho_{4,0}\right)(t)\right]=f_{2}(t),
$$

where $b_{j, 2}(t)$ is the inverse Laplace transform of $B_{j, 2}(s) /\left[h_{2}(s)+\varepsilon B_{2,2}(s)\right]$ and $f_{2}(t)$ is the inverse Laplace transform of $\left\{F_{n}(s)+\varepsilon G_{n}(s)\right\} /\left[h_{2}(s)+\varepsilon B_{2,2}(s)\right]$.

Lemma 7.5. If the functions in (5.33), (5.35) satisfy the regularity conditions $w^{1,0} \in H^{4}\left(B_{R}\right), \rho_{0} \in H^{7 / 2}(\Sigma)$ and $\left\|w^{1,0}\right\|_{H^{4}\left(B_{R}\right)}+\left\|\rho_{0}\right\|_{H^{7 / 2}(\Sigma)}$ is small, then the following inequalities hold: For $n \neq 2$ :

$$
\begin{aligned}
& \left|b_{j, n}(t)\right| \leq C_{0}\left\{(n+1)^{3} e^{-\delta_{1}\left(n^{3}+1\right) t}+(n+1)^{2} e^{-\delta_{1}\left(n^{2}+1\right) t}\right\}, \\
& \left|f_{n}(t)\right| \leq(n+1)^{-3} e^{-\delta_{1} t},
\end{aligned}
$$

and for $n=2$, if $\varepsilon>0$, then

$$
\begin{aligned}
& \left|b_{j, 2}(t)\right| \leq C_{0} e^{-2 \delta_{1}|\varepsilon| t}, \quad j=0,4, \\
& \left|f_{2}(t)\right| \leq e^{-2 \delta_{1}|\varepsilon| t}
\end{aligned}
$$


for some $\delta_{1}>0$. If we drop the assumption that $\left\|w^{1,0}\right\|_{H^{4}\left(B_{R}\right)}+\left\|\rho_{0}\right\|_{H^{7 / 2}(\Sigma)}$ is small, then we need to add a constant factor in (7.11), (7.13).

If we drop the assumption that $\left\|w^{1,0}\right\|_{H^{4}\left(B_{R}\right)}+\left\|\rho_{0}\right\|_{H^{7 / 2}(\Sigma)}$ is small, then we need to add a constant factor in (7.11) and (7.13).

Proof. By examining the structure of $B_{j, n}(s)$ (using also (6.36) ) we find that

$$
\begin{array}{r}
B_{j, n}(s)=M_{j, n}^{1}+M_{j, n}^{2} P_{j}(R \sqrt{s+1})+M_{j, n}^{3} P_{n}(R \sqrt{s+1}) \\
+M_{j, n}^{4}(s+1) P_{j}(R \sqrt{s+1}) P_{n}(R \sqrt{s+1}),
\end{array}
$$

where $M_{j, n}^{m}(m=1,2,3,4,|j-n| \leq 2)$ are constants such that

$$
\begin{aligned}
& \left|M_{j, n}^{1}\right| \leq C(n+1)^{3}, \\
& \left|M_{j, n}^{2}\right|+\left|M_{j, n}^{3}\right| \leq C(n+1), \\
& \left|M_{j, n}^{4}\right| \leq C .
\end{aligned}
$$

Thus [13, Lemma 5.1] can be directly applied to the inverse Laplace transform of $B_{j, n}(s) / h_{n}(s)$ for the first three terms in (7.14). To finish the proof, it suffices to establish that the inverse Laplace transform of

$$
(s+1) P_{j}(R \sqrt{s+1}) P_{n}(R \sqrt{s+1}) / h_{n}(s) \quad \text { for } j=n-2, n, n+2
$$

is bounded by the right-hand side of (7.10). This function is holomorphic for Re $s>-\delta_{1}\left(n^{2}+1\right)$.

From (2.13) we have

$$
\xi^{2} P_{n}(\xi) P_{n+1}(\xi)=1-(2 n+3) P_{n}(\xi), \xi^{2} P_{n+1}(\xi) P_{n+2}(\xi)=1-(2 n+5) P_{n+1}(\xi) .
$$

The quotient of these two equalities gives

$$
\frac{P_{n+2}(\xi)}{P_{n}(\xi)}=\frac{1-(2 n+5) P_{n+1}(\xi)}{1-(2 n+3) P_{n}(\xi)}
$$

which can be rewritten as

$$
P_{n+2}(\xi)-P_{n}(\xi)=(2 n+3) P_{n}(\xi) P_{n+2}(\xi)-(2 n+5) P_{n}(\xi) P_{n+1}(\xi),
$$

and using (2.17) and (2.22), we obtain

$$
\left|P_{n+2}(\xi)-P_{n}(\xi)\right| \leq \frac{C(n+1)}{|\xi|^{2}} \quad \text { for }|\arg (\xi)| \leq \frac{3 \pi}{8} .
$$

We can compute from (2.2), (2.3), and (2.4) that

$$
\left|P_{0}(\xi)-\frac{1}{\xi}\right|+\left|P_{1}(\xi)-\frac{1}{\xi}\right| \leq \frac{C}{|\xi|^{2}} \quad \text { for }|\arg (\xi)| \leq \frac{3 \pi}{8} .
$$

We then deduce by induction (using (7.17)) that

$$
\left|P_{n}(\xi)-\frac{1}{\xi}\right| \leq \frac{C(n+1)^{2}}{|\xi|^{2}} \quad \text { for }|\arg (\xi)| \leq \frac{3 \pi}{8} .
$$

It follows that, for $|n-j| \leq 2$,

$$
\begin{aligned}
\left|P_{j}(\xi) P_{n}(\xi)-\frac{1}{\xi^{2}}\right| & \leq\left|\left(P_{j}(\xi)-\frac{1}{\xi}\right) P_{n}(\xi)\right|+\left|\frac{1}{\xi}\left(P_{n}(\xi)-\frac{1}{\xi}\right)\right| \\
& \leq \frac{C(n+1)^{2}}{|\xi|^{3}} \text { for }|\arg (\xi)| \leq \frac{3 \pi}{8}
\end{aligned}
$$


By repeating the proof of [13, Lemma 5.1] we can replace in (7.15) the function $h_{n}(s)$ with $c_{1} s+c(n)\left(c_{1}\right.$ and $c(n)$ are positive and $c(n) \approx$ const $\cdot n^{3}$ for large $\left.n\right)$, incurring an error that can be estimated by the right-hand side of (7.10).

We write the integral

$$
\frac{1}{2 \pi i} \int_{-\delta_{1}\left(n^{2}+1\right)-i \infty}^{-\delta_{1}\left(n^{2}+1\right)+i \infty} \frac{(s+1) P_{j}(R \sqrt{s+1}) P_{n}(R \sqrt{s+1})}{c_{1} \cdot s+c(n)} e^{s t} d s
$$

as the sum of the integrals for $|\tau| \leq \delta_{1}\left(n^{2}+1\right)$ and $|\tau| \geq \delta_{1}\left(n^{2}+1\right)$. The integral for $|\tau| \leq \delta_{1}\left(n^{2}+1\right)$ is bounded by $C(n+1)^{2} e^{-\delta_{1}\left(n^{2}+1\right) t}$, as seen by using the uniform bounds from (2.16). For the integral on $|\tau| \geq \delta_{1}\left(n^{2}+1\right)$, we replace $R^{2} P_{n}(R \sqrt{s+1}) P_{j}(R \sqrt{s+1})$ by $1 /(s+1)$, incurring an error which is bounded by the right-hand side of (7.20); the resulting error in the integral for $|\tau|>\delta_{1}\left(n^{2}+1\right)$ is bounded by the right-hand side of (7.10) (here we use the fact that if $\xi^{2}=R^{2}(s+1)$ and $s=-\delta_{1}\left(n^{2}+1\right)+i \tau$, then $|\arg (\xi)| \leq \frac{3 \pi}{8}$ if $\left.|\tau| \geq \delta_{1}\left(n^{2}+1\right)\right)$. The integral obtained after this replacement can be computed explicitly, and it is clearly bounded by the right-hand side of (7.10).

We next establish (7.11). Observe that $F_{n}(s)$ and $G_{n}(s)$ are determined by only the initial data $\rho_{n, 0}(0)$, the $\xi_{1 j}^{1}(s)$ from (6.18), the $\psi_{1 j}^{1}(s)$ from (6.20), and $\xi_{1 j}^{2}(s)$ from (6.33). All these functions are uniquely determined by the initial data $w_{n, 0}^{1}(r, 0)$. By applying [12, Lemmas 3.2 and 3.3], we can then estimate all the terms in $F_{n}(s), G_{n}(s)$, except for

$$
\frac{d^{2}}{d r^{2}}\left[-\frac{\mu_{2}}{s+1} \xi_{1 j}^{1}+\psi_{1 j}^{1}\right]_{r=R}
$$

To estimate this term we extend [12, Lemmas 3.2 and 3.3] by repeating their proof with one higher order derivative; this requires one higher order derivative (in $r$ ) on the initial data, which is provided by the regularity assumptions in Lemma 7.5 and [16. Lemma 8.2]. If $n=2$ and $\varepsilon>0$, then $h_{2}(s)+\varepsilon B_{22}(s)=h_{2}^{\prime}(0) s+B_{22}(0) \varepsilon+$ $O\left(|s|^{2}+\varepsilon^{2}\right)$ and we can therefore repeat the previous proof of (77.10), (7.11) and derive the estimate (7.12), (7.13).

\section{Rigorous derivation of (7.8)-(7.9)}

The Laplace transforms performed in Section 6 were carried out in a formal way. In order to justify this procedure we must prove that the solution of (5.24)-(5.35) exists for all $t>0$ and that $\rho$ satisfies, for instance, the inequality

$$
\sup _{0<t<\infty} e^{-\alpha t}\left(\|\rho\|_{H^{7 / 2}(\Sigma)}+\left\|\rho_{t}\right\|_{H^{1 / 2}(\Sigma)}\right)<\infty
$$

for some $\alpha>0$.

In this section we want to derive (7.8)-(7.9) rigorously for a solution of (5.24)(5.35) which is only defined on a finite time interval:

Theorem 8.1. Assume that $w_{0} \in H^{4}\left(B_{R}\right), \rho_{0} \in H^{7 / 2}(\Sigma)$ and let $\left(w_{i}, q_{i}, \rho\right)$ be a solution of (5.24) -(5.35) in a finite time interval $0<t<t_{0}$. Then the formulas (7.8) - (17.9) are valid in the same interval $0<t<t_{0}$.

Proof. Let $T_{1}=t_{0}$ and $T$ be any number smaller than $T_{1}$. We introduce a cut-off function $\zeta \in C^{\infty}[0, \infty)$ such that

$$
\zeta(t) \equiv 1 \quad \text { if } 0<t<T, \quad \zeta(t) \equiv 0 \quad \text { if } T+1<t<\infty
$$


and multiply the system (5.24) by $\zeta(t)$. Setting

$$
\begin{aligned}
& \widetilde{\rho}(\theta, t)=\zeta(t) \rho(\theta, t), \\
& \widetilde{w}_{j}(r, \theta, t)=\zeta(t) w_{j}(r, \theta, t) \quad(j=1,2), \\
& \widetilde{q}_{j}(r, \theta, t)=\zeta(t) q_{j}(r, \theta, t) \quad(j=1,2),
\end{aligned}
$$

we get

$$
\begin{aligned}
& \left(\widetilde{w}_{1}\right)_{t}-\Delta \widetilde{w}_{1}+\widetilde{w}_{1}=k_{1}(r, \theta, t) \quad \text { in } B_{R} \times\{t>0\}, \\
& \left(\widetilde{w}_{2}\right)_{t}-\Delta \widetilde{w}_{2}+\widetilde{w}_{2}=k_{2}(r, \theta, t) \quad \text { in } B_{R} \times\{t>0\}, \\
& \widetilde{w}_{1}=-\frac{\partial \sigma_{S}(R)}{\partial r} \widetilde{\rho} \quad \text { on } \partial B_{R} \times\{t>0\} \\
& \widetilde{w}_{2}=-\left[\frac{\partial \sigma_{1}(R, \theta)}{\partial r}+\frac{\partial^{2} \sigma_{S}(R)}{\partial r^{2}} Y_{2,0}\right] \widetilde{\rho}-\frac{\partial \widetilde{w}_{1}(R, \theta, t)}{\partial r} Y_{2,0} \\
& \quad \text { on } \partial B_{R} \times\{t>0\}, \\
& \Delta \widetilde{q}_{1}=-\mu_{2} \widetilde{w}_{1} \quad \text { in } B_{R} \times\{t>0\}, \\
& \Delta \widetilde{q}_{2}=-\mu_{2} \widetilde{w}_{2}-\mu^{\prime}(0) \widetilde{w}_{1} \quad \text { in } B_{R} \times\{t>0\}, \\
& \widetilde{q}_{1}=-\frac{1}{R^{2}}\left(\widetilde{\rho}+\frac{1}{2} \Delta_{\omega} \widetilde{\rho}\right) \quad \text { on } \partial B_{R} \times\{t>0\}, \\
& \widetilde{q}_{2}=\frac{Y_{2,0}}{R^{3}}(-4 \widetilde{\rho}+\Delta \omega \widetilde{\rho})-\frac{\partial \widetilde{q}_{1}(R, \theta, t)}{\partial r} Y_{2,0} \quad \text { on } \partial B_{R} \times\{t>0\}, \\
& \widetilde{\rho}_{t}=- \\
& \left.\quad-\frac{\partial^{2} p_{S}(R)}{\partial r^{2}}+\varepsilon\left(\frac{\partial^{2} p_{1}(R, \theta)}{\partial r^{2}}+\frac{\partial^{3} p_{S}(R)}{\partial r^{3}} Y_{2,0}\right)\right\} \widetilde{\rho}+k_{3}(\theta, t) \\
& \quad-\left.\frac{\partial \widetilde{q}_{1}}{\partial r}\right|_{r=R}-\varepsilon\left[\frac{\partial \widetilde{q}_{2}}{\partial r}+\frac{\partial^{2} \widetilde{q}_{1}}{\partial r^{2}} Y_{2,0}\right]_{r=R} \\
& \quad+\left.\varepsilon \frac{1}{R^{2}} \frac{\partial Y_{2,0}}{\partial \theta} \frac{\partial \widetilde{q}_{1}}{\partial \theta}\right|_{r=R}+\left.\varepsilon \frac{1}{R^{2}} \frac{\partial \widetilde{\rho}}{\partial \theta} \frac{\partial p_{1}}{\partial \theta}\right|_{r=R},
\end{aligned}
$$

where

$$
k_{j}(r, \theta, t)=\zeta^{\prime}(t) w_{j}(r, \theta, t) \quad(j=1,2), \quad k_{3}(\theta, t)=\zeta^{\prime}(t) \rho(\theta, t) .
$$

We regard $k_{j}$ as inhomogeneous terms and proceed to perform the Laplace transform as in Section 6. We shall only describe here the changes that need to be done in the calculations of Section 6.

In Step (i) from (6.9)-6.14), there are no changes at all.

In Step (ii), an extra term $\widehat{u}_{k_{1}, n}$ due to the inhomogeneous right-hand side should be added to $\widehat{w}_{n, 0}^{1}$ in (6.17); we can find out that $u_{k_{1}, n}$ satisfies:

$$
\begin{aligned}
& \left(u_{k_{1}, n}\right)_{t}-\Delta u_{k_{1}, n}+\left(\frac{n(n+1)}{r^{2}}+1\right) u_{k_{1}, n}=\left\langle k_{1}, Y_{n, 0}\right\rangle \quad \text { in } B_{R}, \\
& u_{k_{1}, n}=0 \quad \text { on } \partial B_{R}, \\
& \left.u_{k_{1}, n}\right|_{t=0}=0
\end{aligned}
$$

and since $k_{1}(r, \theta, t) \equiv 0$ for $0<t<T$, by the maximum principle,

$$
u_{k_{1}, n} \equiv 0 \quad \text { for } 0<t<T \text {. }
$$

This term is carried over to (6.19)-(6.22), resulting in two extra terms in (6.23):

$$
\left[-\frac{\mu_{2}}{s+1} \widehat{u}_{k_{1}, n}+\psi_{k_{1}, n}(s)\right]_{r=R}
$$


where

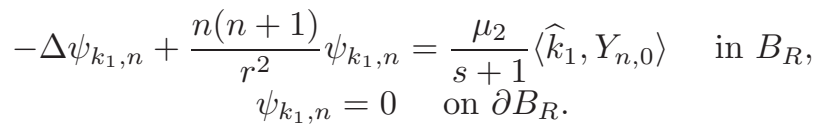

Continuing with the computation, we find that the following extra terms appear in (6.23):

$$
\sum_{j=n-2}^{n+2} c_{j n} \frac{d^{2}}{d r^{2}}\left[-\frac{\mu_{2}}{s+1} \widehat{u}_{k_{1}, n}+\psi_{k_{1}, n}(s)\right]_{r=R}
$$

In Step (iii), a term is added to (6.26) in the form

$$
-\left.\sum_{j=n-2}^{n+2} c_{j n} \frac{d}{d r} \widehat{u}_{k_{1}, n}\right|_{r=R}
$$

All the added terms show up in (6.27)-6.34) through the expression $\widehat{w}_{n, 0}^{2}(R, s)$. Furthermore, an extra term is added to (6.27) due to the inhomogeneous term $k_{2}$; it is $\widehat{u}_{k_{2}, n}$, the Laplace transform of the solution $u_{k_{2}, n}$ :

$$
\begin{aligned}
& \left(u_{k_{2}, n}\right)_{t}-\Delta u_{k_{2}, n}+\left(\frac{n(n+1)}{r^{2}}+1\right) u_{k_{2}, n}=\left\langle k_{2}, Y_{n, 0}\right\rangle \quad \text { in } B_{R}, \\
& u_{k_{2}, n}=0 \quad \text { on } \partial B_{R}, \\
& \left.u_{k_{2}, n}\right|_{t=0}=0
\end{aligned}
$$

and by the maximum principle,

$$
u_{k_{2}, n} \equiv 0 \quad \text { for } 0<t<T .
$$

Altogether we obtain in (6.35) the extra terms

$$
\begin{aligned}
& -\frac{n}{R} \sum_{j=n-2}^{n+2} \frac{d}{d r}\left[\frac{-\mu_{2}}{s+1} \widehat{u}_{k_{1}, j}+\psi_{k_{1}, j}\right]_{r=R} c_{j n} \\
& \quad+\left.\mu_{2} R P_{n}(R \sqrt{s+1}) \sum_{j=n-2}^{n+2} c_{j n} \frac{d}{d r} \widehat{u}_{k_{1}, n}\right|_{r=R} \\
& \quad+\frac{d}{d r}\left[\psi_{k_{2}, n}-\frac{\mu^{\prime}(0)}{s+1} \widehat{u}_{k_{1}, n}\right]_{r=R}-\left.\frac{\mu_{2}}{s+1} \frac{d}{d r} \widehat{u}_{k_{2}, n}\right|_{r=R},
\end{aligned}
$$

where

$$
\begin{gathered}
-\Delta \psi_{k_{2}, n}+\frac{n(n+1)}{r^{2}} \psi_{k_{2}, n}=\frac{\mu^{\prime}(0)}{s+1}\left\langle\widehat{k}_{1}, Y_{n, 0}\right\rangle+\frac{\mu_{2}}{s+1}\left\langle\widehat{k}_{2}, Y_{n, 0}\right\rangle \text { in } B_{R}, \\
\psi_{k_{2}, n}=0 \quad \text { on } \partial B_{R} .
\end{gathered}
$$

Lemma 8.2. The inverse Laplace transforms of $\psi_{k_{1}, n}$ and $\psi_{k_{2}, n}$ are well defined, and if we denote them by $v_{k_{1}, n}(t)$ and $v_{k_{2}, n}(t)$, then

$$
v_{k_{1}, n}(t) \equiv v_{k_{2}, n}(t) \equiv 0 \quad \text { for } 0<t<T .
$$

Proof. Clearly, $v_{k_{1}, n}(t)$ satisfies

$$
\begin{gathered}
-\Delta v_{k_{1}, n}+\frac{n(n+1)}{r^{2}} v_{k_{1}, n}=\mu_{2} e^{-t} *\left\langle k_{1}, Y_{n, 0}\right\rangle \text { in } B_{R}, \\
v_{k_{1}, n}=0 \quad \text { on } \partial B_{R} .
\end{gathered}
$$


From the previous consideration we know that $k_{1}=0$ for $0<t<T$, so that for any function $b(\cdots, t)$

$$
\left(k_{1} * b\right)(t)=\int_{0}^{t} k_{1}(\cdots, \tau) b(\cdots, t-\tau) d \tau=0 \quad \text { if } 0<t<T .
$$

Thus the right-hand side of (8.27) is zero when $0<t<T$. It follows that $v_{k_{1}, n}(t) \equiv$ 0 for $0<t<T$. The proof for $v_{k_{2}, n}$ is similar.

Proof of Theorem 8.1 (continued). The equation for $\rho$ involves $k_{3}$, which can be treated in a similar manner to $k_{1}$ and $k_{2}$. Thus the truncated system (as a result of the cut-off function $\zeta(t)$ ) coincides with the original system for $t<T$, and of course we can take the Laplace transform of this system. We can then proceed as in Section 6 to derive equations as in Lemma 6.1 with several extra terms on the right-hand side of (6.8), given explicitly by (8.18) and (8.24) and a term involving $k_{3}$. We now proceed as in Section 8 to derive (7.8), (7.9) for $\widetilde{\rho}$, with several extra terms on the right-hand side. These terms take the form

$$
u_{k_{j}, n} * b_{j, n} \quad(j=1,2), \quad v_{k_{j}, n} * \widetilde{b}_{j, n} \quad(j=1,2), \quad\left\langle k_{3}, Y_{n, 0}\right\rangle * \bar{b}_{n},
$$

for some functions $b_{j, n}, \widetilde{b}_{j, n}, \bar{b}_{n}$, their $r$ derivatives on the boundary, etc., these terms must vanish on the interval $[0, T]$.

Thus we derived (7.8)-(7.9) from (5.24)-(5.35) without the assumption that the solution is global in time.

\section{LineAR INSTABILITY FOR $\varepsilon<0$}

It is proved in [12, Section 4] that if $\mu=\mu_{2}$ (which occurs when $R>\bar{R}$ ), then the roots for $h_{n}(s)$ for $n \neq 1,2$ lie in Re $s<0$. Thus any possible instability in the system (6.8) may arise only from the modes $n=1,2$.

It is also proved in [12, (4.5),(3.27)] that $h_{1}(0)=h_{2}(0)=0$, and except for $s=0$, all remaining roots of $h_{1}$ and $h_{2}$ lie in $\operatorname{Re} s<0$. It is then clear from the structure of (6.8) that the $\varepsilon$-order terms in modes $n=1,2$ will decide the stability.

We recall from [12, Lemma 4.7] that

$$
\frac{d h_{2}(0)}{d s}>0 .
$$

If $\varepsilon<0$, then for the initial data as in Lemma 6.3, the function

$$
A(s)=\frac{1}{h_{2}(s)+\varepsilon B_{2,2}(s)}
$$

will have a zero at

$$
s=-\varepsilon \frac{B_{2,2}(0)}{h_{2}^{\prime}(0)}+O\left(\varepsilon^{2}\right) \equiv \delta_{0}(\varepsilon)>0,
$$

and we therefore expect linear instability. In fact we shall prove the following:

Theorem 9.1. If $\varepsilon<0$, then, for the initial data as in (7.5), there exists a solution of (5.24) - (5.33) for which

$$
\left|\rho_{2,0}\left(t_{n}\right)\right| \rightarrow \infty \text { for a sequence } t_{n} \rightarrow \infty ;
$$

hence the bifurcation branch with $\varepsilon<0$ is linearly unstable. 
Proof. Denote by $\mathcal{K}$ the metric space of sequences $\left\{v_{n}(s)\right\}_{n=0,2,4, \cdots}$ of functions holomorphic in $\left\{\left|s-\delta_{0}(\varepsilon)\right|>\frac{1}{2} \delta_{0}(\varepsilon)\right.$, Re $\left.s>0\right\}$, satisfying

$$
\begin{aligned}
& \left|v_{2}(s)-A(s)\right| \leq \frac{|\varepsilon|^{-1 / 2}}{1+|s|^{2}} \quad \text { if }\left|s-\delta_{0}(\varepsilon)\right| \geq \frac{1}{2} \delta_{0}(\varepsilon), \operatorname{Re} s>0, \\
& \left|v_{n}(s)\right| \leq \frac{|\varepsilon|^{(n-5) / 3}}{1+|s|^{2}} \quad \text { if }\left|s-\delta_{0}(\varepsilon)\right| \geq \frac{1}{2} \delta_{0}(\varepsilon), \text { Re } s>0, n \geq 4, \\
& \left|v_{0}(s)\right| \leq \frac{|\varepsilon|^{-1 / 3}}{1+|s|^{2}} \quad \text { if }\left|s-\delta_{0}(\varepsilon)\right| \geq \frac{1}{2} \delta_{0}(\varepsilon), \text { Re } s>0,
\end{aligned}
$$

with the distance between two sequences $\left\{v_{n}(s)\right\}_{n=0,2,4, \cdots}$ and $\left\{\bar{v}_{n}(s)\right\}_{n=0,2,4, \cdots}$ defined by

$$
\begin{aligned}
& \sup _{\left\{\left|s-\delta_{0}(\varepsilon)\right| \geq \frac{1}{2} \delta_{0}(\varepsilon),\right.}\{\operatorname{sep} s>0\}_{n \geq 4}|\varepsilon|^{-(n-5) / 3}\left(1+|s|^{2}\right)\left|v_{n}(s)-\bar{v}_{n}(s)\right|, \\
&\left.|\varepsilon|^{-1 / 2}\left(1+|s|^{2}\right)\left|v_{2}(s)-\bar{v}_{2}(s)\right|,|\varepsilon|^{-1 / 3}\left(1+|s|^{2}\right)\left|v_{0}(s)-\bar{v}_{0}(s)\right|\right\} .
\end{aligned}
$$

We introduce a mapping $\mathcal{M}$ from $\left\{v_{n}(s)\right\}_{n=0,2,4, \cdots}$ to $\left\{u_{n}(s)\right\}_{n=0,2,4, \cdots}$ by

$$
\begin{aligned}
& u_{2}(s)-A(s)=-\varepsilon\left\{\frac{B_{0,2}(s) v_{0}(s)}{h_{2}(s)+\varepsilon B_{2,2}(s)}+\frac{B_{4,2}(s) v_{4}(s)}{h_{2}(s)+\varepsilon B_{2,2}(s)}\right\}, \\
& h_{n}(s) u_{n}(s)=-\varepsilon \sum_{j=n-2}^{n+2} B_{j, n}(s) v_{j}(s), \quad n \neq 2 .
\end{aligned}
$$

We shall prove that $\mathcal{M}$ is a contraction mapping in $\mathcal{K}$, and it thus has a unique fixed point, which is then the unique solution of (7.6) in $\mathcal{K}$.

As proved in [12, Section 4] for $n \neq 2,\left|h_{n}(s)\right| \geq c(n)$ in the region $\left\{\left|s-\delta_{0}(\varepsilon)\right| \geq\right.$ $\frac{1}{2} \delta_{0}(\varepsilon)$, Re $\left.s>0\right\}$, where $c(n)$ is positive and $c(n) \approx$ const.$n^{3}$ for large $n$. If $n \geq 6$, the function $v_{2}(s)$ does not appear in (9.7), and therefore

$$
\begin{aligned}
\left|u_{n}(s)\right| & =|\varepsilon|\left|\sum_{j=n-2}^{n+2} \frac{1}{h_{n}(s)} B_{j, n}(s) v_{j}(s)\right| \\
& \leq|\varepsilon| C \sum_{j=n-2}^{n+2} \frac{|\varepsilon|^{(j-5) / 3}}{1+|s|^{2}} \leq \frac{|\varepsilon|^{(n-7) / 3}}{1+|s|^{2}} \cdot C|\varepsilon| \\
& <\frac{|\varepsilon|^{(n-5) / 3}}{1+|s|^{2}} \text { if }\left|s-\delta_{0}(\varepsilon)\right| \geq \frac{1}{2} \delta_{0}(\varepsilon), \text { Re } s>0,
\end{aligned}
$$

if $|\varepsilon|$ is small such that $C|\varepsilon|^{1 / 3}<1$.

By (9.2), (9.3), on $\left|s-\delta_{0}(\varepsilon)\right|=\frac{1}{2} \delta_{0}(\varepsilon)$ we have $1 / A(s)=h_{2}^{\prime}(0) s+\varepsilon B_{2,2}(0)+$ $O\left(\varepsilon^{2}\right) \sim$ const $\cdot|\varepsilon|$ as $\varepsilon \rightarrow 0$, so that

$$
\begin{aligned}
\left|v_{2}(s)\right| \leq\left|v_{2}(s)-A(s)\right|+ & |A(s)|<\frac{|\varepsilon|^{-1 / 2}}{1+|s|^{2}}+C \frac{|\varepsilon|^{-1}}{1+|s|}<C \frac{|\varepsilon|^{-1}}{1+|s|} \\
& \text { for }\left|s-\delta_{0}(\varepsilon)\right| \geq \frac{1}{2} \delta_{0}(\varepsilon), \operatorname{Re} s>0 .
\end{aligned}
$$

Using this inequality, noticing also that $\left|h_{4}(s)\right| \geq c(1+|s|)$ for $\left\{\left|s-\delta_{0}(\varepsilon)\right| \geq\right.$ $\left.\frac{1}{2} \delta_{0}(\varepsilon), \operatorname{Re} s>0\right\}$, we obtain the same estimates for $u_{4}(s)$ as in (9.8). 
In the case $n=0$, we can use (7.7), (9.9) and $\left|h_{0}(s)\right| \geq c(1+|s|)$ for $\left\{\left|s-\delta_{0}(\varepsilon)\right| \geq\right.$ $\left.\frac{1}{2} \delta_{0}(\varepsilon), \operatorname{Re} s>0\right\}$ to deduce from (9.7) that

$$
\begin{aligned}
\left|u_{0}(s)\right| & \leq C|\varepsilon|\left\{\frac{|\varepsilon|^{-1 / 3}}{1+|s|^{2}}+\frac{|\varepsilon|^{-1}}{1+|s|^{2}}\right\} \\
& \leq \frac{C}{1+|s|^{2}}<\frac{|\varepsilon|^{-1 / 3}}{1+|s|^{2}} \quad \text { for }\left|s-\delta_{0}(\varepsilon)\right| \geq \frac{1}{2} \delta_{0}(\varepsilon), \operatorname{Re} s>0 .
\end{aligned}
$$

Finally, for $n=2$, notice that the right-hand side of (9.7) does not involve $v_{2}$, whereas $v_{0}$ and $v_{4}$ are bounded by $|\varepsilon|^{-1 / 3} /\left(1+|s|^{2}\right)$. Hence

$$
\begin{aligned}
\left|u_{2}(s)-A(s)\right| & \leq \frac{C}{\left|h_{2}(s)+\varepsilon B_{2,2}(s)\right|} \frac{|\varepsilon|^{2 / 3}}{1+|s|^{2}} \leq \frac{C}{|\varepsilon|} \frac{|\varepsilon|^{2 / 3}}{1+|s|^{2}} \\
& \leq \frac{|\varepsilon|^{-1 / 2}}{1+|s|^{2}} \quad \text { for }\left|s-\delta_{0}(\varepsilon)\right| \geq \frac{1}{2} \delta_{0}(\varepsilon), \operatorname{Re} s>0 .
\end{aligned}
$$

We have thus proved that $\mathcal{M}$ maps $\mathcal{K}$ into itself. The proof that $\mathcal{M}$ is a contraction follows by a similar argument. We conclude that $\mathcal{M}$ has a unique fixed point in $\mathcal{K}$, and this is the unique solution of (7.6) in the region $\left|s-\delta_{0}(\varepsilon)\right| \geq \frac{1}{2} \delta_{0}(\varepsilon)$, Re $s>0$.

The inverse Laplace transform of $A(s)$ is well defined, as shown in 12. The estimate (9.5) allows us to define the inverse Laplace transform for the functions $u_{2}(s)-A(s)$ and $u_{n}(s)(n \neq 2)$ and these are holomorphic in Re $s>a$ for any $a>\delta_{0}(\varepsilon)$.

We can then define the inverse Laplace transform (with Re $s=a, a>\delta_{0}(\varepsilon)$ ) of $\hat{\rho}$, and then define $w_{1}, w_{2}, q_{1}, q_{2}$ as solutions of the equations (5.24)-(5.31). As in [12, we can then prove that $(w, q, \rho)$ is the solution of the linearized problem (5.24) - (5.32). We now make the simple but important observation that if $\rho$ is bounded, then its Laplace transform $\widehat{\rho}$ is a holomorphic function in $\operatorname{Re} s>0$ and so are the elements in the sequence $\left\{\widehat{\rho}_{n, 0}\right\}_{n=0,2,4, \ldots}$ of its expansion into a series of spherical harmonics. Since $\left\{\widehat{\rho}_{n, 0}\right\}_{n=0,2,4, \ldots}$ must agree with the fixed point on the circle $\left|s-\delta_{0}(\varepsilon)\right|=\frac{1}{2} \delta_{0}(\varepsilon)$,

$$
\begin{aligned}
& \left|\widehat{\rho}_{2,0}(s)-A(s)\right| \leq|\varepsilon|^{-1 / 2} \quad \text { if }\left|s-\delta_{0}(\varepsilon)\right|=\frac{1}{2} \delta_{0}(\varepsilon), \\
& \left|\widehat{\rho}_{n, 0}(s)\right| \leq|\varepsilon|^{(n-5) / 3} \quad \text { if }\left|s-\delta_{0}(\varepsilon)\right|=\frac{1}{2} \delta_{0}(\varepsilon), n \geq 4, \\
& \left|\widehat{\rho}_{n, 0}(s)\right| \leq|\varepsilon|^{-1 / 3} \quad \text { if }\left|s-\delta_{0}(\varepsilon)\right|=\frac{1}{2} \delta_{0}(\varepsilon), n=0,2 .
\end{aligned}
$$

Recalling that $A(s) \sim$ const $|\varepsilon|$ on $\left|s-\delta_{0}(\varepsilon)\right|=\frac{1}{2} \delta_{0}(\varepsilon)$,

$$
\left|\widehat{\rho}_{2,0}(s)-A(s)\right| \leq|\varepsilon|^{-1 / 2}<|A(s)| \quad \text { on }\left|s-\delta_{0}(\varepsilon)\right|=\frac{1}{2} \delta_{0}(\varepsilon),
$$

and this implies that inside the disk $\left|s-\delta_{0}(\varepsilon)\right|<\frac{1}{2} \delta_{0}(\varepsilon)$,

$$
\begin{aligned}
& \text { (number of poles - number of roots) of } \widehat{\rho}_{2,0}(s) \\
& \quad=(\text { number of poles }- \text { number of roots) of } A(s)=1 .
\end{aligned}
$$

Thus $\widehat{\rho}_{2,0}(s)$ has one pole $\alpha+i \beta$ with $\alpha>0$, which contradicts the assumption that $\rho(t)$ is a bounded function.

This establishes the assertion (9.4). 


\section{LinEAR STABILITY FOR $\varepsilon>0$}

We consider the case $\varepsilon>0$, and assume that

$$
\left\|w^{1,0}\right\|_{H^{4}\left(B_{R}\right)}+\left\|\rho_{0}\right\|_{H^{7 / 2}(\Sigma)} \text { is finite. }
$$

To prove linear stability, it suffices to consider two separate cases: (i) the perturbation functions are even functions of $\theta$, and (ii) the perturbation functions are odd functions of $\theta$.

Theorem 10.1. If $\varepsilon>0$, and the initial data are even function in $\theta$ satisfying (10.1), then there exists a unique solution of (5.24)-(5.33) such that

$$
\begin{aligned}
& \left|\rho_{n, 0}(t)\right| \leq C(n+1)^{-3} e^{-\delta_{1}|\varepsilon| t} \quad \text { for } n \neq 2, \\
& \left|\rho_{2,0}(t)\right| \leq C e^{-\delta_{1}|\varepsilon| t},
\end{aligned}
$$

where $C$ is a constant independent of $\varepsilon$.

Proof. As in the proof of Theorem 9.1 it suffices to prove that there exists a unique solution of (7.8), (7.9) satisfying (10.2)-(10.3). Without loss of generality we may assume that the expression in (10.1) is sufficiently small so that (7.10), (7.11) hold; we shall then prove that

$$
\begin{aligned}
& \left|\rho_{n, 0}(t)\right| \leq(n+1)^{-3} e^{-\delta_{1}|\varepsilon| t} \quad \text { for } n \neq 2, \\
& \left|\rho_{2,0}(t)\right| \leq K e^{-\delta_{1}|\varepsilon| t}
\end{aligned}
$$

where $K=1+2 C_{0} / \delta_{1}$ and $C_{0}$ is the constant which appears in (17.10). The proof is again based on the contraction mapping principle. Let $\mathcal{K}$ be the set of sequences

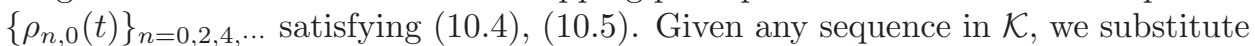
it into the $\varepsilon$-order terms in (7.8), (7.9) and solve for a new $\widetilde{\rho}_{n, 0}$, thus defining a mapping

$$
\left\{\widetilde{\rho}_{n, 0}(t)\right\}_{n=0,2,4, \cdots}=\mathcal{M}\left\{\rho_{n, 0}(t)\right\}_{n=0,2,4, \cdots} .
$$

Using Lemma 7.5, we have, for $n \neq 2$,

$$
\begin{aligned}
\left|\widetilde{\rho}_{n, 0}(t)\right| \leq & \left|f_{n}(t)\right|+|\varepsilon| \sum_{j=n-2}^{n+2}\left|\left(b_{j, n} * \rho_{j, 0}\right)(t)\right| \\
& \leq(n+1)^{-3} e^{-\delta_{1} t}+|\varepsilon| \sum_{j=n-2}^{n+2} 2 C_{0}\left\{(n+1)^{3} e^{-\delta_{1}\left(n^{3}+1\right) t}\right. \\
& \left.\quad+(n+1)^{2} e^{-\delta_{1}\left(n^{2}+1\right) t}\right\} *\left\{(n+1)^{-3}\left(1+3^{3} K\right) e^{-\delta_{1}|\varepsilon| t}\right\} \\
& \leq(n+1)^{-3} e^{-\delta_{1} t}+2 C_{0}\left(1+3^{3} K\right)|\varepsilon|(n+1)^{-3} e^{-\delta_{1}|\varepsilon| t} \\
& <2(n+1)^{-3} e^{-\delta_{1}|\varepsilon| t},
\end{aligned}
$$

provided we choose $2 C_{0}\left(1+3^{3} K\right)|\varepsilon|<1$.

For $n=2$, we use (7.9) to obtain

$$
\begin{aligned}
\left|\widetilde{\rho}_{2,0}(t)\right| & \leq\left|f_{2}(t)\right|+2 C_{0}|\varepsilon| e^{-2 \delta_{1}|\varepsilon| t} * e^{-\delta_{1}|\varepsilon| t} \\
& \leq e^{-2 \delta_{1}|\varepsilon| t}+2 C_{0}|\varepsilon| \frac{1}{\delta_{1}|\varepsilon|} e^{-\delta_{1}|\varepsilon| t} \\
& <K e^{-\delta_{1}|\varepsilon| t}
\end{aligned}
$$


provided we choose $K=1+2 C_{0} / \delta_{1}$. This proves that $\mathcal{M}$ maps $\mathcal{K}$ into itself. If we define the distance between $\left\{\rho_{n, 0}\right\}_{n=0,2,4, \cdots}$ and $\left\{\widetilde{\rho}_{n, 0}\right\}_{n=0,2,4, \ldots}$ by

$$
\begin{gathered}
\max \left\{\sup _{n \neq 2} \sup _{0 \leq t<\infty}(n+1)^{3} e^{\delta_{1}|\varepsilon| t \mid}\left|\rho_{n, 0}(t)-\widetilde{\rho}_{n, 0}(t)\right|,\right. \\
\left.\sup _{0 \leq t<\infty} K^{-1} e^{\delta_{1}|\varepsilon| t}\left|\rho_{2,0}(t)-\widetilde{\rho}_{2,0}(t)\right|\right\},
\end{gathered}
$$

we can then repeat the above argument to show that we have a contraction on this space, and hence there is a unique fixed point for $\mathcal{M}$, which gives a unique solution in $\mathcal{K}$.

If $|\varepsilon|$ is small, then the solution of the system (7.8)-(7.9) for $0<t<1$ is unique not only in the space of functions $\rho$ satisfying (10.2), (10.3) for $0<t<1$, but also in the larger space of functions $\rho(t, \theta)$ with

$$
\int_{0}^{1}\|\rho\|_{L^{2}(\Sigma)}^{2} d t<\infty
$$

Since a solution (5.24) -(5.35) in $0<t<1$ must satisfy (7.8) -(7.9) (by Theorem 8.1), we obtain the uniqueness assertion of Theorem 10.1 for $0<t<1$. We can now proceed to prove uniqueness for $1 \leq t \leq 2$ (with the same $\varepsilon$ ), $2 \leq t \leq 3$, etc.

We next consider the system odd in $\theta$. In this case we only need to consider the system for $n=1,3,5, \cdots$.

Theorem 10.2. Let $\varepsilon>0$ and let the initial data be odd functions in $\theta$ satisfying $w^{1,0} \in H^{4}\left(B_{R}\right)$ and $\rho_{0} \in H^{7 / 2}(\Sigma)$. Then there exists a point $x_{0}$, depending only on the initial data, such that for the system obtained analogously to (5.24)-(5.35) by linearization about $x_{0}$, there exists a unique solution satisfying (10.2), (10.3).

Proof. The idea of the proof is essentially the same as in [13, Section 7], but the details are much simpler since here we are dealing with a linear system and not with the product of solutions from the nonlinear deformation. We only outline the proof.

Recall that the roots of $h_{2 n+1}(s)$ lie in the half plane $\operatorname{Re} s<0$, while $h_{1}(0)=0$ and all the other roots of $h_{1}(s)$ lie in Re $s<0$. In order to circumvent the difficulty caused by the zero root of $h_{1}(s)$, we need to make a translation of the center about which we linearize. We have dealt with such a problem already in 12, where we considered the stability of the nonlinear problem (1.1)-(1.6) with respect to the stationary radial solution. The present case is much easier since we are dealing with a linearized problem.

We rewrite the system (6.8) as

$$
h_{n}(s) \widehat{\rho}_{n, 0}(s)=F_{n}(s)+\varepsilon G_{n}(s)-\varepsilon \sum_{j=n-2}^{n+2} B_{j, n}(s) \widehat{\rho}_{j, 0}(s) ;
$$

in particular,

$$
h_{1}(s) \widehat{\rho}_{1,0}(s)=F_{1}(s)+\varepsilon G_{1}(s)-\varepsilon f(s),
$$

where

$$
f(s)=\sum_{j=1}^{3} B_{j, 1}(s) \widehat{\rho}_{j, 0}(s)
$$


Notice that $F_{1}(s)$ and $G_{1}(s)$ depend only on the initial data, but $f(s)$ does not depend explicitly on the initial data.

We shall again use two fixed point theorems as in [13, Section 7]. We look for a solution in the class

$$
\left|\rho_{n, 0}(t)\right| \leq n^{-3} e^{-\delta_{1} t},
$$

where we choose $\delta_{1}$ such that all the roots of $h_{n}(s)$ for $n \geq 3$ and for $h_{1}(s)$ (except for $s=0)$ lie in $\operatorname{Re} s<-2 \delta_{1}$.

Suppose $f(s)$ is a given holomorphic function, uniformly bounded in a neighborhood of $s=0$. By applying [13, Theorem 2.2] we can make a translation $x \rightarrow x+a$ of the center of the linearization; this translation is equivalent to keeping the origin fixed, but translating the initial data according to [13, (6.4), (6.5)].

The functions on the right-hand side of (10.7) actually depend on $a$, through their dependence on the initial data according to [13, (6.4),(6.5)], and for clarity we shall write the right-hand side of (10.8) as

$$
F_{1}(s ; a)+\varepsilon G_{1}(s ; a)-\varepsilon f(s) .
$$

We want to solve for $a$ such that

$$
F_{1}(0 ; a)+\varepsilon G_{1}(0 ; a)-\varepsilon f(0)=0 .
$$

This can be done, as in [13, recalling that $F_{1}(0, a)$ is given by [13, (6.3)] (when $\varepsilon=0$ ) and using a fixed point theorem [13, Theorem 2.2].

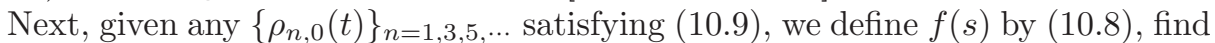
$a$ by (10.10), and use this choice of $a$ into $F_{n}$ and $G_{n}$ of (10.6).

We then proceed to solve the new $\widetilde{\rho}_{n, 0}(t)$ as the inverse Laplace transform of $\widehat{\widetilde{\rho}}_{n, 0}(s)$ (which is defined by the right-hand side of (10.6)). Since (10.10) cancels the zero of $h_{1}(s)$ at $s=0$, we can proceed as in the proof Theorem 10.1 to obtain the new $\widetilde{\rho}_{n, 0}(t)$ satisfying (10.9). We can further prove that the map $\mathcal{M}:\left\{\rho_{n, 0}(t)\right\} \rightarrow$ $\left\{\widetilde{\rho}_{n, 0}(t)\right\}$ is a contraction. Thus we have a fixed point, which corresponds to a solution that converges to zero exponentially fast. For the even terms coming out of this translation of the center (they must be of order $\varepsilon^{2}$ or higher, by 13 , $(6.4),(6.5)])$, we apply Theorem 10.1. Uniqueness follows as in Theorem 10.1

Remark 10.1. Our method of proving linear stability can be extended to allow functions depending also on $\phi$. However, in this case, we cannot separate the odd and even modes, and we are forced to combine the proofs of Theorems 10.1 and 10.2, which makes the computation more complicated.

\section{Hopf BIFURCATION: LineARIZED PROBLEM}

In this section we consider the case $R_{S}<\bar{R}$. In this case $\mu^{*}\left(R_{S}\right)=\mu_{0}^{*}\left(R_{S}\right)<$ $\mu_{2}^{*}\left(R_{S}\right)$ [12. We consider the linearized problem about the radially symmetric solution by setting

$$
\begin{aligned}
& \sigma(r, \theta, \phi, t)=\sigma_{S}(r)+\varepsilon w(r, \theta, \phi, t), \\
& p(r, \theta, \phi, t)=p_{S}(r)+\varepsilon q(r, \theta, \phi, t), \\
& \partial \Omega(t): r=R_{S}+\varepsilon \rho(\theta, \phi, t),
\end{aligned}
$$


and using the relations

$$
\begin{aligned}
& V_{n}=\varepsilon \rho_{t}+O\left(\varepsilon^{2}\right), \\
& \kappa=\frac{1}{R}-\frac{\varepsilon}{R^{2}}\left(\rho+\frac{1}{2} \Delta_{\omega} \rho\right)+O\left(\varepsilon^{2}\right) .
\end{aligned}
$$

Substituting these quantities into (1.1) - 1.5) and collecting only the linear terms in $\varepsilon$, we obtain, as in 12 , the linearized system

$$
\begin{aligned}
& w_{t}-\Delta w+w=0 \quad \text { in } B_{R} \times\{t>0\}, \\
& w(R, \theta, \phi, t)=-\lambda \rho(\theta, \phi, t) \quad \text { for } t>0 \quad\left(\lambda=\left.\frac{\partial}{\partial r} \sigma_{S}(r)\right|_{r=R}\right), \\
& \Delta q=-\mu w \quad \text { in } B_{R} \times\{t>0\}, \\
& q(R, \theta, \phi, t)=-\frac{1}{R^{2}}\left(\rho+\frac{1}{2} \Delta_{\omega} \rho\right),
\end{aligned}
$$

and

$$
\frac{d \rho}{d t}=-\left.\frac{\partial^{2} p_{S}}{\partial r^{2}}\right|_{r=R} \rho-\left.\frac{\partial q}{\partial r}\right|_{r=R} \quad \text { for } t>0
$$

Using (1.7) and (1.8) we find that

$$
\left.\frac{\partial^{2} p_{S}}{\partial r^{2}}\right|_{r=R}=-\mu(1-\tilde{\sigma})
$$

Thus the equation for $\rho$ can also be written in the form

$$
\frac{d \rho}{d t}=\mu(1-\tilde{\sigma}) \rho-\left.\frac{\partial q}{\partial r}\right|_{r=R} \quad \text { for } t>0 .
$$

From (1.9) we also have the initial conditions

$$
\left.w\right|_{t=0}=w_{0}(r, \theta, \phi) \quad \text { for } 0 \leq r \leq R,\left.\quad \rho\right|_{t=0}=\rho_{0}(\theta, \phi) .
$$

Theorem 11.1. For $R<\bar{R}$ and $\mu=\mu_{0}^{*}(R)$,

(i) there exists a family of periodic solutions of the form:

$$
\rho(\theta, \phi, t)=\rho(t)=C_{1} \sin \beta t+C_{2} \cos \beta t+\sum_{m=-1}^{1} D_{m} Y_{1, m}(\theta, \phi)
$$

for the linearized problem, where $\pm i \beta$ are roots of $h_{0}(s)$, and $\beta>0$;

(ii) any periodic solution to the linearized problem is of the form (11.8);

(iii) for any initial data $w_{0} \in L^{2}\left(B_{R}\right), \rho_{0} \in L^{2}(\Sigma)$, the solution of the linearized problem converges exponentially fast to a periodic solution established in (i), (ii).

Proof. We first prove existence. Since the systems are linear, we can consider each mode separately. We look for solutions of the form

$$
\begin{aligned}
& \rho(t, \theta, \phi)=e^{\nu t} Y_{n, m}(\theta, \phi), \\
& w(r, \theta, \phi, t)=e^{\nu t} u(r) Y_{n, m}(\theta, \phi), \\
& q(r, \theta, \phi, t)=e^{\nu t} \zeta(r) Y_{n, m}(\theta, \phi),
\end{aligned}
$$


where

$$
\begin{aligned}
& -\Delta u+\left(\frac{n(n+1)}{r^{2}}+\nu+1\right) u=0 \quad \text { in } B_{R} \\
& u=-\lambda \quad \text { on } \partial B_{R}, \\
& -\Delta \zeta+\frac{n(n+1)}{r^{2}} \zeta=\mu u \quad \text { in } B_{R} \\
& \zeta=\frac{1}{R^{2}}\left(\frac{n(n+1)}{2}-1\right) \quad \text { on } \partial B_{R} .
\end{aligned}
$$

One can verify that this is a solution if and only if $h_{n}(\nu)=0$. For $n=0$, under our assumptions, we have $h_{0}( \pm i \beta)=0$ for some $\beta>0$. Substituting into (11.9) we obtain (i).

We next establish convergence and uniqueness. The Laplace transform has been computed in [12, Section 3], or equivalently, we can use the formulas in Section 5 by setting $q_{2} \equiv w_{2} \equiv 0$; here, however, we shall allow our functions to depend on $(r, \theta, \phi, t)$. Since for $n \geq 2$ all the roots of $h_{n}(s)$ lie in $\operatorname{Re} s<0$, for mode $n \geq 2$, all solutions converge to zero exponentially fast, and therefore there are no periodic solutions with these modes.

Consider next the modes $n=0,1$. We have

$$
\widehat{\rho}_{n, m}=\frac{1}{\mu R^{2} P_{0}(R) h_{n}(s)} F_{n, m}(s) \quad(-n \leq m \leq n),
$$

where

$$
F_{n, m}(s)=\rho_{n, m}(0)+\frac{d}{d r}\left[\frac{\mu}{s+1} \xi_{1, n, m}-\psi_{1, n, m}\right]_{r=R}
$$

and $\xi_{1, n, m}(r, s), \psi_{1, n, m}(r, s)$ are the solutions of

$$
\begin{gathered}
-\Delta \xi_{1, n, m}+\left(\frac{n(n+1)}{r^{2}}+(s+1)\right) \xi_{1, n, m}=w_{n, m}(r, 0) \quad \text { in } B_{R}, \\
\left.\xi_{1, n, m}\right|_{r=R}=0,
\end{gathered}
$$

and

$$
\begin{gathered}
-\Delta \psi_{1, n, m}+\frac{n(n+1)}{r^{2}} \psi_{1, n, m}=\frac{\mu}{s+1} w_{n, m}(r, 0) \quad \text { in } B_{R}, \\
\left.\psi_{1, n, m}\right|_{r=R}=0 .
\end{gathered}
$$

For $n=0$, except for $\pm i \beta(\beta>0)$, all other roots of $h_{0}(s)$ lie in $\operatorname{Re} s<-2 \delta_{1}$. Thus

$$
\begin{aligned}
\rho_{0,0}(t)= & \frac{1}{2 \pi i} \int_{a-i \infty}^{a+i \infty} \frac{1}{\mu R^{2} P_{0}(R) h_{0}(s)} F_{0,0}(s) e^{s t} d s \quad(a>0) \\
= & \left(\operatorname{Res}_{s=\beta i}+\operatorname{Res}_{s=-\beta i}\right) \frac{F_{0,0}(s) e^{s t}}{\mu R^{2} P_{0}(R) h_{0}(s)} \\
& \quad+\frac{1}{2 \pi i} \int_{-\delta_{1}-i \infty}^{-\delta_{1}+i \infty} \frac{1}{\mu R^{2} P_{0}(R) h_{0}(s)} F_{0,0}(s) e^{s t} d s \\
\equiv & \rho_{P}(t)+J(t) .
\end{aligned}
$$

Since $h_{0}(s)$ has simple roots at $s= \pm i \beta, \rho_{P}(t)$ is of the form $\widetilde{C}_{1} e^{i \beta t}+\widetilde{C}_{2} e^{-i \beta t}$ for some complex constants $\widetilde{C}_{1}$ and $\widetilde{C}_{2}$. Since our solution is real, we must have

$$
\rho_{P}(t)=C_{1} \sin \beta t+C_{2} \cos \beta t
$$


for some real constants $C_{1}$ and $C_{2}$. As in the proof of [12, Section 5], $J(t)$ converges to 0 exponentially fast. Hence

$$
\left|\rho_{0,0}(t)-\rho_{P}(t)\right| \leq C e^{-\delta_{1} t} \quad \text { for } t>0 .
$$

Let

$$
\begin{aligned}
& w_{P}(r, t)=\rho_{P}(t) u(r), \\
& q_{P}(r, t)=\rho_{P}(t) \zeta(r),
\end{aligned}
$$

where

$$
\begin{aligned}
& -\Delta u+(\omega+1) u=0 \quad \text { in } B_{R}, \\
& u=-\lambda \quad \text { on } \partial B_{R}, \\
& -\Delta \zeta=\mu u \quad \text { in } B_{R}, \\
& \zeta=-\frac{1}{R^{2}} \quad \text { on } \partial B_{R} .
\end{aligned}
$$

Then the function $W=w_{0,0}(r, t)-w_{P}(r, t)$ and $Q=q_{0,0}(r, t)-q_{P}(r, t)$ satisfy the system

$$
\begin{aligned}
& W_{t}-\Delta W=W=0 \quad \text { in } B_{R} \times\{t>0\}, \\
& W(R, t)=-\lambda\left[\rho_{0,0}(t)-\rho_{P}(t)\right] \quad \text { for } t>0, \\
& \Delta Q=-\mu W \quad \text { in } B_{R} \times\{t>0\}, \\
& Q(R, t)=-\frac{1}{R^{2}}\left[\rho_{0,0}(t)-\rho_{P}(t)\right] .
\end{aligned}
$$

Using parabolic estimates we can then also prove that

$$
\left|w_{0,0}(r, t)-w_{P}(r, t)\right|+\left|q_{0,0}(r, t)-q_{P}(r, t)\right| \leq C e^{-\delta_{1} t} \quad \text { for } t>0 .
$$

We can use a similar argument for the mode $n=1$; in this case $s=0$ is a simple root for $h_{1}(s)$ and all other roots lie in $\operatorname{Re} s<-2 \delta_{1}$. The above procedure shows that all solutions in mode 1 must converge exponentially fast to $\sum_{m=-1}^{1} D_{m} Y_{1, m}(\theta, \phi)$, for some constants $D_{m}$.

Remark 11.1. The Hopf bifurcation for a nonlinear system with a parameter $\mu$ produces a family of periodic solutions for $\mu=\mu(\varepsilon)$ in a neighborhood of the bifurcation point $\mu=\mu_{*}\left(\mu_{*}=\mu(0)\right)$. However, for a linear problem, such as the one considered in Theorem 11.1] periodic solutions (as $t \rightarrow \infty$ ) can arise only when $\mu$ coincides with the bifurcation point $\mu_{*}$.

\section{ACKNOWLEDGEMENT}

This work was partially supported by the National Science Foundation upon agreement No. 0112050.

\section{REFERENCES}

[1] B. Bazally and A. Friedman, A free boundary problem for an elliptic-parabolic system: Application to a model of tumor growth, Comm. Partial Diff. Eq., Vol. 28, 2003, pp. 517-560. MR:1976462 (2004c:35420)

[2] H.M. BYRNE, The importance of intercellular adhesion in the development of carcinomas, IMA J. Math. Appl. Med. Biol., Vol. 14, 1997, pp. 305-323.

[3] H.M. Byrne, A weakly nonlinear analysis of a model of avascular solid tumour growth, J. Math. Biol., Vol. 39, 1999, pp. 59-89. MR1705626(2000i:92011) 
[4] H.M. Byrne And M.A.J. Chaplain, Modelling the role of cell-cell adhesion in the growth and development of carcinomas, Mathl. Comput. Modelling, Vol. 12, 1996, pp. 1-17.

[5] H.M. Byrne and M.A.J. Chaplain, Growth of nonnecrotic tumors in the presence and absence of inhibitors, Math. Biosci., Vol. 130, 1995, pp. 151-181.

[6] H.M. Byrne And M.A.J. Chaplain, Growth of nonnecrotic tumors in the presence and absence of inhibitors, Math. Biosci., Vol. 135, 1996, pp. 187-216.

[7] M.A.J. Chaplain, The development of a spatial pattern in a model for cancer growth, Experimental and Theoretical Advances in Biological Pattern Formation (H.G. Othmer, P.K. Maini, and J.D. Murray, eds), Plenum Press, 1993, pp. 45-60.

[8] X. Chen And A. Friedman, A free boundary problem for an elliptic-hyperbolic system: An application to tumor growth, SIAM J. Math. Anal., Vol. 35, 2003, pp. 974-986. MR2049029 (2005f:35333)

[9] M.G. Crandall and P.H. Rabinowitz, Bifurcation from simple eigenvalies, J. Functional Analysis, Vol. 8, 1971, pp. 321-340. MR0288640 (44:5836)

[10] M. Fontelos And A. Friedman, Symmetry-breaking bifurcations of free boundary problems in three dimensions, Asymptotic Analysis, Vol. 35, 2003, pp. 187-206. MR2011787 (2005a:35290)

[11] M. Fontelos And A. Friedman, Symmetry-breaking bifurcations of charged drops, Arch. Rat. Mech. Anal., Vol. 172, 2004, pp. 267-294. MR2058166 (2005b:76140)

[12] A. Friedman and Bei Hu, Bifurcation from stability to instability for a free boundary problem arising in a tumor model, Arch. Rat. Mech. Anal., Vol. 180, No. 2, 2006, pp. 293-330. MR2210911 (2006j:35246)

[13] A. Friedman And Bei Hu, Asymptotic stability for a free boundary problem arising in a tumor model, J. Diff. Eqn., Vol. 227, No. 2, 2006, pp. 598-639. MR.2237681

[14] A. Friedman and F. Reitich, Analysis of a mathematical model for the growth of tumors, J. Math. Biology, Vol. 38, 1999, pp. 262-284. MR1684873 (2001f:92011)

[15] A. Friedman And F. Reitich, Symmetry-breaking bifurcation of analytic solutions to free boundary problems: An application to a model of tumor growth, Trans. Amer. Math. Soc., Vol. 353, 2001, pp. 1587-1634. MR1806728 (2002a:35208)

[16] A. Friedman and F. Reitich, Nonlinear stability of a quasi-static Stefan problem with surface tension: A continuation approach, Ann. Scoula Norm. Sup. Pisa Cl. Sci., Vol. 30(4), 2001, pp. 341-403. MR1895715 (2003e:35326)

[17] A. Galindo and P. Pascual, Quantum Mechanics, Vol. I, Springer, New York, 1990. MR:1079543(91k:81001)

[18] H.P. Greenspan, Models for the growth of a solid tumor by diffusion, Studies Appl. Math, Vol. 52, 1972, pp. 317-340.

[19] H.P. Greenspan, On the growth and stability of cell cultures and solid tumors, J. Theoretical Biology, Vol. 56, 1976, pp. 229-242. MR0429164 (55:2183)

Department of Mathematics, The Ohio State University, 231 West 18th Avenue, Columbus, Оhiо 43210

E-mail address: afriedman@mbi.osu.edu

Department of Mathematics, University of Notre Dame, Notre Dame, Indiana 46556

E-mail address: b1hu@nd.edu 